\title{
IMPROVING REDUCED VARIABLE MODELS FOR COMPLEX SYSTEMS VIA EXPERIMENT
}

\author{
A Dissertation \\ Presented to \\ the faculty of the School of Engineering and Applied Science \\ University of Virginia
}

\author{
In partial fulfillment \\ of the requirements for the Degree \\ of Doctor of Philosophy in Chemical Engineering
}

Karen Blaha 


\section{APPROVAL SHEET}

The dissertation is submitted in partial fulfillment of the requirements for the degree of

Doctor of Philosophy in Chemical Engineering

AUTHOR

This dissertation has been read and approved by the examining Committee:

Dissertation Advisor

Accepted for the School of Engineering and Applied Science:

Dean, School of Engineering and Applied Science

Dec 2013 


\section{Abstract}

Populations of simple interacting oscillators can give rise to complex dynamics. Real-world examples of such systems abound in biology, chemistry, and physics. Reduced-variable models can describe these systems. The phase model is a particularly successful reduced-variable model which, in its simplest form, each element is represented by one variable, the phase of oscillation. The phase model can be constructed from observable variables, without specific knowledge of underlying phenomenological behavior. Such reduced variable models are easier to construct and more generalizable than models derived from underlying physics or chemistry. In this dissertation, we study the dynamics of a population of coupled electrochemical oscillators in order to modify the phase model to expand its applicability. Specifically, we develop a two-phase model, a phase and radius model, and models with network coupling.

We analyze data from two coupled oscillators with a standard one-dimensional phase model and a newer two-dimensional phase model. The same quantity of data is needed for either model. The two-dimensional analysis reveals behaviors and coupling parameters in theoretical and experimental examples that the onedimensional analysis does not show. The two-dimensional model could be useful in systems that exhibit learning due to its ability to distinguish stimulation and reaction. 
We examine clustering on small networks with time-delayed interactions. Our experimental results agree with numerical and analytical results from a phase and amplitude model (the Stuart-Landau model). For near-sinusoidal oscillators, the standard Stuart-Landau model successfully describes clustering dynamics. We extend the Stuart-Landau model to describe clustering dynamics for higher harmonic oscillators. We present examples of asymmetrical clusters arising from networks of higher harmonic oscillators. We show that the amplitude of oscillation is functionally influenced by coupling; we believe this "amplitude coupling function" has not been previously described. This function can be constructed from the original time series with no additional measurements.

Recently, combined phase and amplitude models have gained attention; we explore the conditions where a phase and amplitude model is useful. We show experimentally a change in cluster state due only to changes in coupling strength. Such a transition is not possible with a phase-only model. Simulations with the extended Stuart-Landau model match experimental results. We demonstrate a method for predicting the amplitude coupling function from the coupling. Prior to this study, the relationship between stimulation and response was not known for the amplitude. We suggest that the phase and amplitude model will be most useful (1) in modeling high-harmonic oscillations, and (2) where coupling exceeds the "weak coupling" approximation of the phase-only model. 


\section{Acknowledgments}

I would like to thank my parents for their patience and their support over the years. My father I thank specifically for his editing assistance, and for my introduction to chemical engineering. I thank my brother for my first interest in science, after his lies about the black holes under the bed, and my introduction to chaos, though the lessons were cut far too short.

I thank Professor Hudson for his guidance and wisdom over the years, and the honor of being his last student. I would like to thank our collaborators Prof. Michael Rosenblum, Prof. Arkady Pikovsky, Prof. István Kiss, Prof. Eckehard Schöll, Phillip Hövel, Thomas Dahms, Judith Lehnert and Andrew Keane. I would like to thank the other members of the Hudson lab over the years: Sarah Johnson, Craig Rusin, Matt Clark, Swati Jain, Joe Basconi, and especially Xin Ren.

I thank my husband and my parents-in-law for their support. I thank my friends from the engineering school, the water polo team, and the science fiction group for support, stimulating conversations, and a wonderful stay in Charlottesville.

This work was funded through NSF and the Love scholarship. 


\section{Contents}

1 Introduction and Scope 1

2 Background $\quad 6$

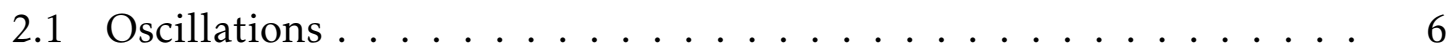

2.1.1 Smooth oscillations ............... 8

2.1.2 Relaxation oscillations .............. 8

2.2 Phase Models . . . . . . . . . . . . . . . . 10

2.3 Time series analysis . . . . . . . . . . . . . . . 12

2.3.1 Peak and phase finding . . . . . . . . . . 12

2.3.2 Finding the the radius .................. 13

2.4 Network Theory . . . . . . . . . . . . . . . . . . 14

2.4.1 The Master Stability Function . . . . . . . . . . . . . 17

3 Experimental Apparatus $\quad 20$

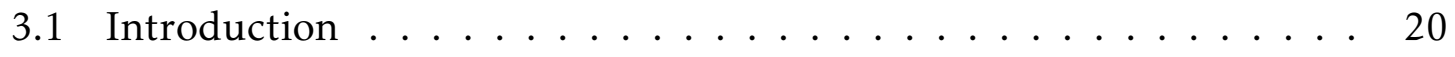

3.2 Electrochemical system . . . . . . . . . . . . . 20

3.2.1 Experimental System . . . . . . . . . . . . 21

3.2.2 Electrode preparation .................. 22

3.2.3 Experimental procedure ............... 24

3.3 Simulation and analysis methods . . . . . . . . . . 27 
4 Two Dimensional Phase Modeling 28

4.1 Notes to the reader . . . . . . . . . . . . . 28

4.2 Introduction . . . . . . . . . . . . . . . 29

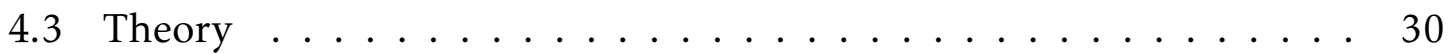

4.4 Experimental setup $\ldots \ldots \ldots \ldots \ldots$. . . . . . . . . . . 34

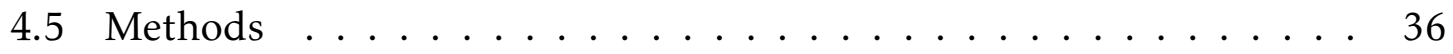

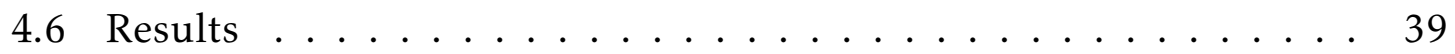

4.7 Discussion ........................... 44

4.8 Acknowledgements ..................... 47

5 Clustering in small networks with delay 48

5.1 Notes to the reader . . . . . . . . . . . . . . 48

5.2 Introduction . . . . . . . . . . . . . . . 49

5.3 Theoretical model: Sinusoidal Oscillations _. . . . . . . . 50

5.4 Experimental setup . . . . . . . . . . . . . . 54

5.5 Smooth oscillations ........................ 58

5.6 Interaction functions ................. 61

5.7 Relaxation oscillations ................. 64

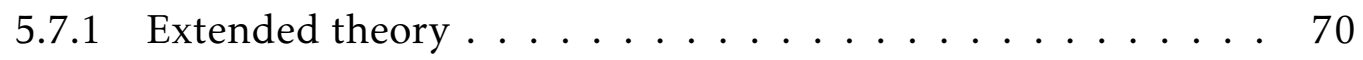

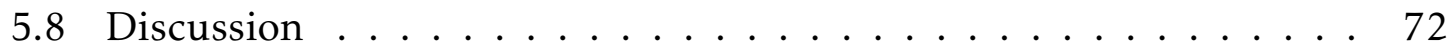

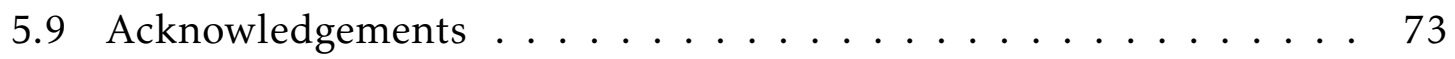

$\begin{array}{lll}6 & \text { Exploring radial dynamics } & 74\end{array}$

6.1 Notes to the reader . . . . . . . . . . . . . . . . 74



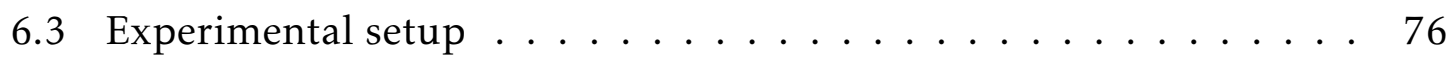

6.4 Theory ................................ 79 
6.5 Results . . . . . . . . . . . . . . . . . 81

6.5.1 Cluster transitions due to coupling strength . . . . . . . 82

6.5.2 Predicting the Radial Interaction Function . . . . . . . . . 85

6.6 Discussion . . . . . . . . . . . . . . . 87

6.7 Acknowledgments . . . . . . . . . . . . . . . 88

$\begin{array}{llr}7 & \text { Other investigations } & 89\end{array}$

7.1 Introduction . . . . . . . . . . . . . . . 89

7.2 Chimeras ......................... 89

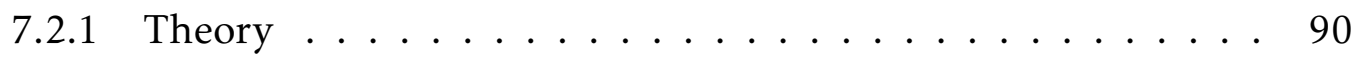

7.2.2 Experimental Methods . . . . . . . . . . . . . . . . . 91

7.2.3 Results and Discussion . . . . . . . . . . . . . . . 93

7.3 Exploring cluster states on more complex networks . . . . . . . . 95

7.3 .1 Theory . . . . . . . . . . . . . . . 95

7.3.2 Experimental Methods and Results . . . . . . . . . . . 96

7.4 Radial effects in the conformist/contrarian system _. . . . . . 100

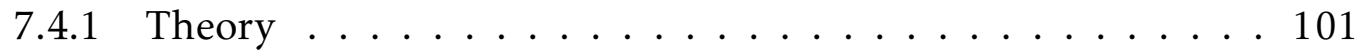

7.4.2 Experimental Methods . . . . . . . . . . . . . . 102

7.4.3 Results and Discussion . . . . . . . . . . . . . 103

8 Conclusions and Future Work $\quad 105$

8.1 Conclusions . . . . . . . . . . . . . . . . . 105

8.2 Future Work . . . . . . . . . . . . . . . . . . . 107

A MATLAB Code $\quad 111$

A.1 Time series analysis $\ldots \ldots \ldots \ldots \ldots \ldots \ldots \ldots$

A.1.1 Peak finder . . . . . . . . . . . . . . 111 
A.1.2 phase_calculator .................. 114

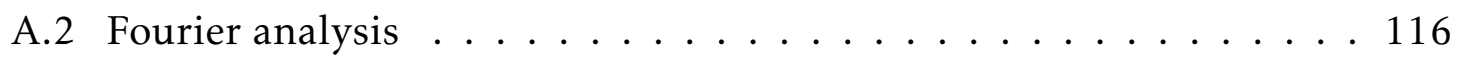

A.2.1 Program to resample data set: even_sample_2pi . . . . . . 116

A.2.2 Program to find $\mathrm{ffts}: \mathrm{fft}_{\mathrm{f}}$ full . . . . . . . . . . 118

A.2.3 Program to find iffts: inv fft . . . . . . . . . . . 119

A.3 A sample full analysis of data . . . . . . . . . . . 120

A.3.1 all_calc ......................... 120

A.4 Preparing a coupling matrix for labview . . . . . . . . . 127

A.4.1 Create_Labview_MTX_File . . . . . . . . . . . . 127

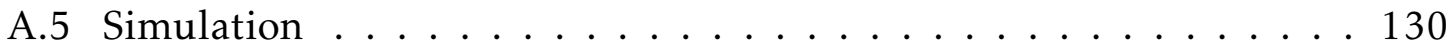

A.5.1 A sample simulator . . . . . . . . . . . . . . 130

$\begin{array}{ll}\text { References } & 136\end{array}$ 


\section{List of Figures}

1.1 A schematic representation of the work in the dissertation. . . . . . 2

2.1 Schematic of the phase portrait of a simple oscillator . . . . . . . 7

2.2 Schematic of the pitchfork bifurcation. . . . . . . . . 8

2.3 Schematic of the Hopf bifurcation. . . . . . . . . . . . 8

2.4 Time series of smooth oscillations. . . . . . . . . . . . . . 9

2.5 Time series of relaxation oscillations. . . . . . . . . . . . . 9

2.6 A schematic representation of the response function $\ldots \ldots . . . .11$

2.7 Finding phase from peak-to-peak linear interpolation. . . . . . . . 13

2.8 Finding the radius. . . . . . . . . . . . . . 13

2.9 A schematic of a simple network and its corresponding connectivity

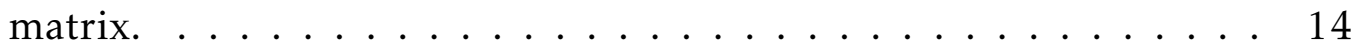

2.10 Schematics and connectivity matrixes for global coupling and coupling in a unidirectional ring. . . . . . . . . . . . . 15

2.11 The master stability function for the Rössler oscillator under several coupling schemes. ....................... 18

3.1 The bifurcation digram for nickel electrodissolution at $\left|\mathrm{H}_{2} \mathrm{SO}_{4}\right|=3.0 \mathrm{M} 22$

3.2 Photos of the electrode array fabrication. . . . . . . . . . . . . 24

3.3 Schematic and photo of the experimental apparatus. . . . . . . 25 
3.4 Photo of the experimental apparatus. . . . . . . . . . . 25

4.1 Synchronization tongues for toy model generated by the one- and two-dimensional models. . . . . . . . . . . . . . . . 33

4.2 Experimental apparatus for the two-phase study. . . . . . . . . 34

4.3 Hilbert space representations of smooth and relaxation oscillators. . 37

4.4 The protophase, the phase transformation function, and the genuine phase in the two-phase method. . . . . . . . . . . . . 37

4.5 One-dimensional coupling functions with and without delay. . . . . 39

4.6 Two-dimensional coupling functions with and without delay. . . . . 40

4.7 A series of two-dimensional coupling functions showing changes in

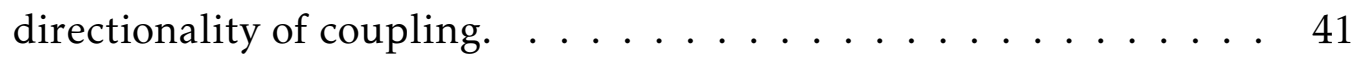

4.8 Relating the norm of the two-dimensional coupling function to the

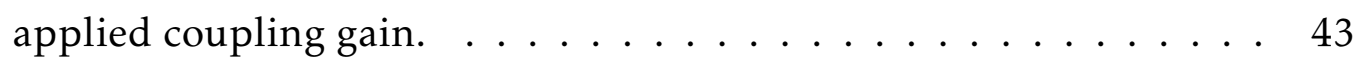

4.9 The two-dimensional coupling function versus the one-dimensional coupling function when the system is driven by a sinusoidal signal

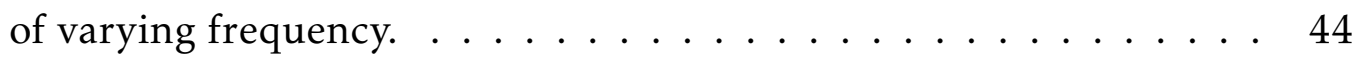

5.1 Theoretically predicted frequencies for primary cluster states . . . 53

5.2 Experimental apparatus for Chapter 5. . . . . . . . . . . . 55

5.3 Cluster and frequency dynamics for smooth oscillators (experimental, simulation, and analytical) as time delay is varied. . . . . . . . . 59

5.4 Time series and schematics of the four primary cluster states which occur on a unidirectional ring of four smooth oscillators. . . . . . 60

5.5 Frequency and radial coupling functions for smooth and relaxation experimental oscillators. . . . . . . . . . . . . 62 
5.6 Cluster and frequency dynamics for relaxation oscillators (experimental, simulation, and analytical) as time delay is varied. . . . . . 66

5.7 Time series and schematics of the four secondary cluster states which occur on a unidirectional ring of four relaxational oscillators. . . . 67

5.8 Cluster and frequency dynamics for relaxation oscillators (experimental, simulation, and analytical) as time delay is varied over a different range. . . . . . . . . . . . . . . .

6.1 (a) Experimental apparatus with multi-channel addressable feedback, $R_{p}$ is the channel resistance of $650 \Omega$. (b) Time series of relaxation oscillators at $V_{0}=1.2 \mathrm{~V}$. ZRA: zero resistance ammeter. $\ldots \ldots 77$

6.2 Phase and radius coupling functions for a relaxation oscillator . . . 81

6.3 Schematics of a cluster state for experiment and simulation . . . . 83

6.4 Radii and phase differences of a cluster state with incremented cou-



6.5 Phase and radius interaction functions for a relaxation oscillator with

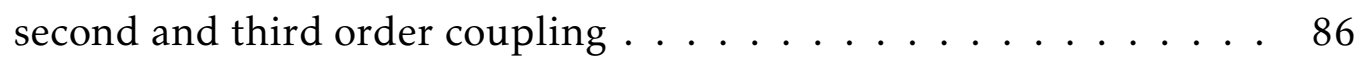

6.6 Experimental versus predicted interaction functions with multi-order coupling $\ldots \ldots \ldots \ldots \ldots \ldots \ldots \ldots$

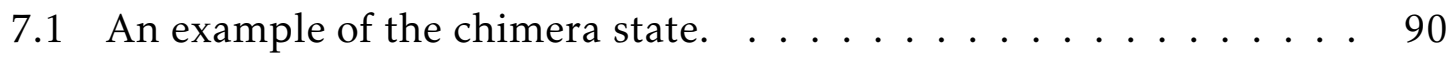

7.2 The bifurcation diagram for a chimera in the presence of heterogeneity. 94

7.3 Time series and schematic of the six primary cluster states observed in the six-member unidirectional ring coupling network. . . . . . 97

7.4 Schematics of six secondary cluster states observed on a six-member

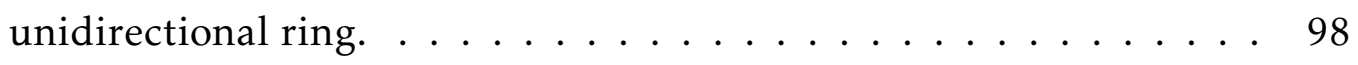


7.5 The experimental cluster progression on a unidirectional six-member ring of relaxation oscillators as $\tau$ is varied. . . . . . . . . . . . . . 99

7.6 Experimental and simulation results with the one-value-weighted unidirectional ring. . . . . . . . . . . . . . . . 99

7.7 Schematic representation and coupling matrix for conformist/contrarian coupling. . . . . . . . . . . . . . 100

7.8 States observed by Hong and Strogatz in the conformist-contrarian

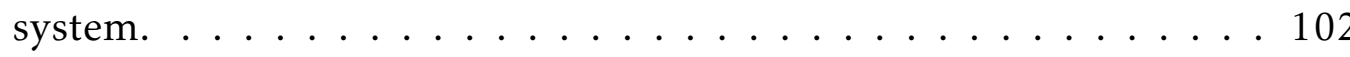

7.9 Amplitude dependence of the conformists and contrarians as the percentage of conformists is varied. . . . . . . . . . . 104 


\section{List of Tables}

$5.1 \quad H_{r}$ and $H_{\varphi}$ Fourier coefficients used in Chapter $5 \ldots \ldots 5$

$6.1 H_{r}$ and $H_{\varphi}$ Fourier coefficients used in Chapter $6 \ldots \ldots 2$

6.2 Standard deviations in reference $H_{r}$ and $H_{\varphi}$ functions $\ldots \ldots$. . . 86 


\section{List of Symbols}

A Amplitude

G Coupling matrix or network

$g_{j, n} \quad$ Element of coupling matrix

$H(\Delta \phi)$ Interaction or coupling function

$H_{\phi} \quad$ Phase coupling function

$H_{r} \quad$ Radial coupling function

$h \quad$ Stimulation function

$I(t) \quad$ Current

$K \quad$ Linear feedback or coupling gain

$N \quad$ Number of elements

P Period

$Q\left(\phi_{1}, \phi_{2}\right)$ Two-dimensional coupling function

$q\left(\phi_{2}-\phi_{1}\right)$ One-dimensional coupling function

$r \quad$ radius 
$s \quad$ Harmonic of coupling

$t \quad$ Time

V Voltage

$V_{0} \quad$ Applied baseline voltage

$x(t) \quad$ Potential drop across the double layer

$Z(\phi)$ Response function

$\alpha \quad$ Phase lag

$\omega \quad$ Natural frequency

$\phi \quad$ Phase

$\tau \quad$ Feedback or coupling delay 


\section{Chapter 1}

\section{Introduction and Scope}

Many natural systems are comprised of coupled oscillating elements. Extensive studies have been performed to understand, predict, and engineer behaviors such as synchrony in these oscillatory populations. Examples include coupled lasers [1], population dynamics [2], chemical reactions [3], superconducting Josephson junctions [4], and cardiorespiratory interactions [5, 6], among others.

If the elements or interactions in a system are nonlinear, populations can exhibit highly complex behavior. The sum is greater than the parts; small changes can be magnified to yield radically different behaviors. Consequently, when we study these systems, we must examine them as a whole, not from the standpoint of individual pieces. Each oscillator can be thought of as an $M$-dimensional problem. If we have a population of $N$ oscillators, this quickly becomes an unwieldy system. In order to study the system, we employ a reduced-variable model called the phase model.

There are several reasons for working with the phase model. If we have $N$ oscillators, the phase model requires only $N$ equations, rather than $N x M$. The phase model is also more intuitive than the full equations, due to its simplicity. Additionally, the phase model lets us compare the dynamics of dissimilar systems 
(such as a biological and a physical system) in the same framework. Finally, the full $M$ dimensional equations are not known for all systems. The phase model can be constructed for any system that has oscillatory elements, because it is constructed only from observable measurements. For example, in our system, oscillations are due to the formation and dissolution of nickel oxide on the surface of an electrode; a full model would need to consider the density of various chemical species present on the electrode. Instead, we construct phase models from the current measured at each electrode. The phase model approach has been employed in a variety of contexts [7-12]. It has been used in biology to model plasticity and learning [13] and inter-neuron coupling [14]. It has been used in physics to model phase synchrony in chaotic lasers [15] and to extract coupling directionality in electronic oscillators [16].



Figure 1.1: Three extensions to the standard phase model: (A) The twophase model, with dynamics depending on two phases rather than a phase difference, (B) the phase and amplitude model, with dynamics depending upon the amplitude in addition to the phase, and (C) the network model, in which all pairwise interactions are scaled by a network, G. $\phi$ denotes phase while $r$ denotes radius/ amplitude.

Previous work in this lab (and the vast majority of the work in the field) has employed the standard phase model [17-20]. This dissertation extends the standard 
phase model in three ways. We represent the dissertation graphically in Fig. 1.1, with the standard phase model depending upon phase difference at the top, and the three extensions to the model given in $\mathrm{A}, \mathrm{B}$, and C.

1. In the two-phase model, interactions are a function of two phases, rather than the phase difference (see Fig. 1.1A). See Chapter 4.

2. In the phase and amplitude model, amplitude dynamics are considered in addition to phase dynamics (see Fig. 1.1B). See Chapters 5 and 6.

3. In the network model, pairwise interactions between elements may now differ in value from one another (see Fig. 1.1C). See Chapters 5 and 6.

The two-phase model and the phase and amplitude model both give more detailed descriptions of the systems than the standard phase model. These extensions can be considered second-order approximations, which may be needed in cases where the first-order approximation of the standard phase model is inadequate. The network model will be needed when the system in question is coupled via a network. All three extensions can be used in combination; in principle it is possible to use all three together. The only additional data needed to employ these three extensions is knowledge of the coupling network, and only in the case of the network model.

In Chapter 2, the background chapter, we introduce the reader to oscillators and the phase model. We describe in detail how the quantities are calculated for the phase model from an oscillatory time series. We briefly describe network theory concepts and the master stability function.

Chapter 3 describes the experimental system and experimental procedure. We detail the experimental apparatus. We describe simulations used in tandem with experiments. 
Chapter 4 compares two-dimensional and one-dimensional phase models for two coupled oscillators, respectively called the two-phase and phase-difference models. The two-phase model may be necessary in cases of more complex dynamics [21]; similar data analysis techniques have been used to measure synchrony in epileptic brain signals [22]. The two-phase model can also be useful for systems where coupling evolves over time, due to the ability to separate stimulation and response $[13,23]$. We demonstrate that model selection is more nuanced than previously thought. Prior to our study, the one-dimensional model was considered adequate for similar oscillators while the two-dimensional model was believed only necessary for dissimilar oscillators. Instead the underlying dynamics dictate the appropriateness of the model. We successfully apply the one-dimensional analysis to dissimilar oscillators and show another case where the two-phase model better describes interactions between similar oscillators. This chapter closely mirrors a paper that we published in 2011 with Michael Rosenblum and Arkady Pikovsky from the University of Potsdam (see Section A.5.1).

Chapter 5 explores clustering on small, delay-coupled networks using experiments, simulations, and the master stability function. There is general interest in small networks as a stepping stone for understanding the dynamics on larger networks [24, 25]. We successfully model dynamics using the Stuart-Landau model, a model which incorporates the phase and radius (amplitude). We show asymmetrical clusters in experiments with higher harmonics.We present a set of modified Stuart-Landau equations to capture system behavior. We show the the amplitude of oscillation is functionally dependant upon the coupling in the "amplitude coupling function", which we believe is a first. This chapter closely mirrors our recently accepted paper with Eckehard Schoell's group at the Technical University in Berlin (see Section A.5.1). 
Chapter 6 discusses the implications of the phase and radius model from Chapter 5 . We show a cluster transition due to changes in coupling strength. The phase and radius model describes this behavior well, unlike the phase-only model. We describe a method to predict amplitude dynamics if the coupling is known, which we believe is a first. We suggest two settings in which a phase and amplitude description may be superior to a phase-only description: (1) When higher harmonics are present, and (2) when coupling exceeds the "weak coupling" requirement of the phase-only model [8]. Higher harmonic oscillators may be more sensitive to the amplitude of perturbation; neurons exhibit a threshold of sensitivity for firing, as do neuron models such as the FitzHugh-Nagumo model [26, 27].

Chapter 7 briefly summarizes other miscellaneous studies. We discuss spatiallydependent coupling which may produce a chimera state, a mix of order and disorder. We discuss amplitude dynamics in systems with two subpopulations. We discuss clustering dynamics on small weighted networks, in which not all pair-wise interactions are equal in magnitude.

Chapter 8 briefly summarizes the dissertation, and describes its place in the field. We discuss possible future work in the field arising from this dissertation, and the broader future of the field. 


\section{Chapter 2}

\section{Background}

Systems of coupled oscillators exist in many fields of study. Each element has some repeated action, and interacts with other elements in some way. The repeated action can be intermittent, such as the firing of a neuron, or highly periodic, such as the swinging of a metronome. Oscillation shape can vary from neuron-spiking to simple sine wave harmonics. Interactions can be unidirectional, bidirectional or weighted, and for different length scales. Small interactions between elements can lead to surprising population behaviors.

\subsection{Oscillations}

As oscillations are central to this dissertation, this section defines them in detail. We are particularly interested in smooth and relaxation oscillations that are periodic. Both kinds of oscillations can be characterized by their phase and radius. The radius is similar to the amplitude of the oscillation-it is the difference between the peak of the waveform and the mean.

Any signal that oscillates periodically can be considered a "limit cycle". Fig. 2.1 shows how a simple pendulum can be considered as either a time series or a limit 



Figure 2.1: A system which oscillates, such as a pendulum (left), can be considered as an oscillatory time series (middle) or as a "limit cycle" in phase space (right) [28].

cycle in phase space. In this view, the oscillation is a circle, and the radius of the oscillation is the radius of this circle.

Limit cycle oscillations often arise as the result of a supercritical Hopf bifurcation. A simplified model for a Hopf bifurcation is

$$
\begin{aligned}
& \dot{r}=\left(\lambda-r^{2}\right) r \\
& \dot{\phi}=\omega,
\end{aligned}
$$

where $r$ is the radius, $\phi$ is the phase, and $\omega$ is the frequency. $\lambda$ is the bifurcation parameter. Readers familiar with nonlinear dynamics may recognize the radial equation as a generic pitchfork bifurcation. As shown in Fig. 2.2, when $\lambda<0$, a single stable point will exist at $r=0$. When $\lambda>0$, the solution $r=0$ becomes unstable, and a pair of stable solutions at $r= \pm \sqrt{\lambda}$ are born.

The phase is always increasing at a rate of $\omega$, thus we can imagine that when $\lambda>0$, the system traces a limit cycle with period $2 \pi / \omega$ and radius $\sqrt{\lambda}$, as shown in Fig. 2.3. The time series of a limit cycle as shown in the figure will oscillate between values of $\pm \sqrt{\lambda}$. 


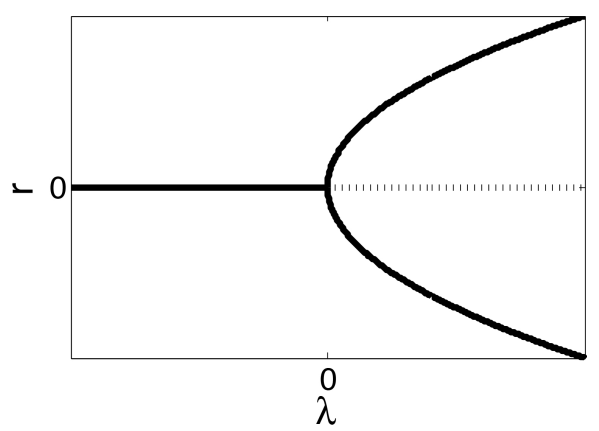

Figure 2.2: The pitchfork bifurcation, as given by Eq. (2.1). Solid lines indicate stable solutions while dashed lines indicate unstable solutions.

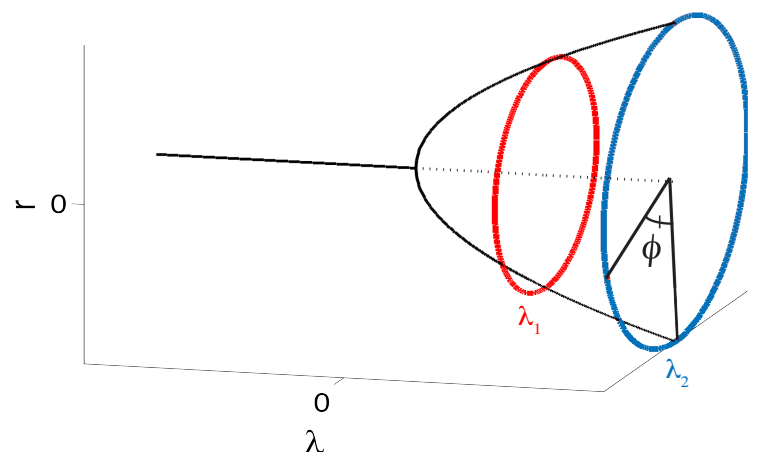

Figure 2.3: A Hopf bifurcation, as given by Eqs. (2.1) and (2.2). Two possible limit cycles for oscillations are shown, at $\lambda_{1}$ (red) and $\lambda_{2}$ (blue). Solid lines indicate stable solutions while dashed lines indicate unstable solutions.

\subsubsection{Smooth oscillations}

Sinusoidal or nearly-sinusoidal oscillations are often called smooth oscillations. These oscillations occur at values of $\lambda$ near the Hopf bifurcation (at $\lambda=0$ ). They have the simplest oscillatory dynamics, and in some cases may be adequately modeled with linear dynamics [8]. Fig. 2.4 shows an example of a smooth electrochemical oscillation.

\subsubsection{Relaxation oscillations}

As we increase the value of the bifurcation parameter, $\lambda$, higher harmonics not captured by the simplified model of Eq. (2.1) appear. These higher harmonics 


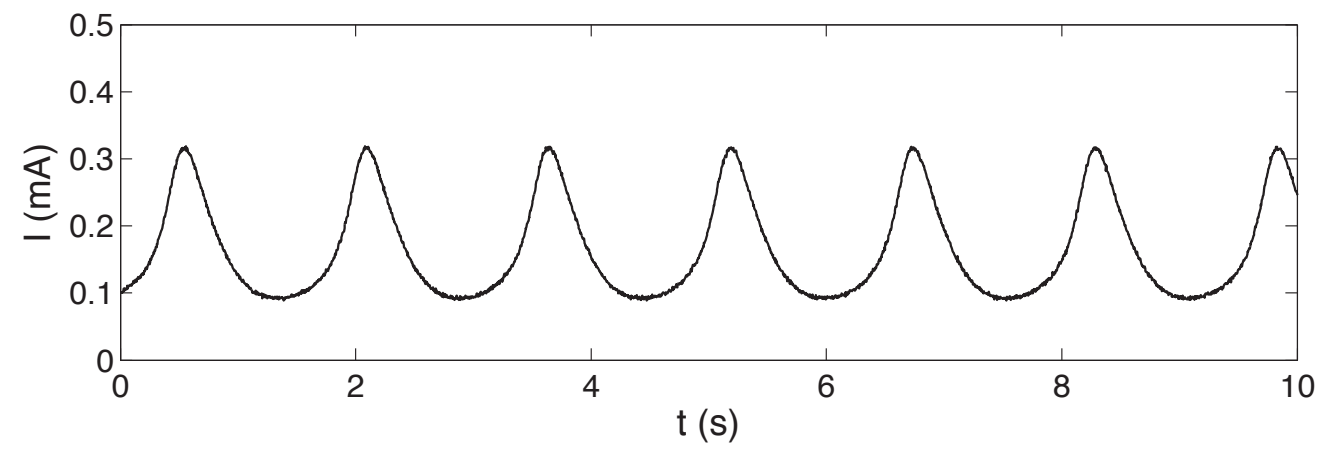

Figure 2.4: Time series of smooth oscillations.

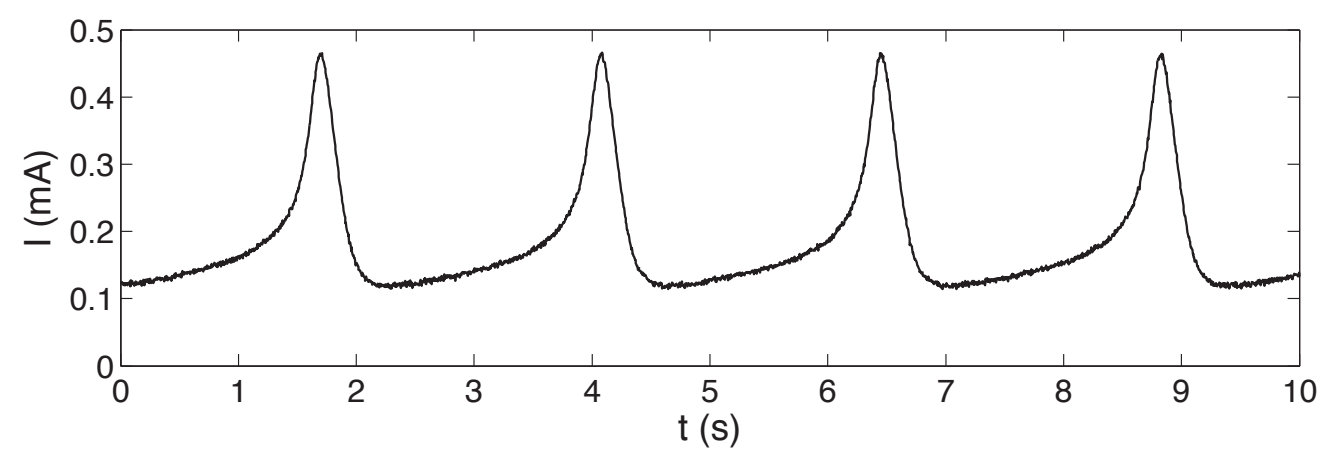

Figure 2.5: Time series of relaxation oscillations.

cause the system to spend more time in certain values of the oscillation; these relaxation oscillators are sometimes called slow-fast oscillators [29]. The phase $\phi$ still increases uniformly by definition. Relaxation oscillations are periodic, and thus can be expressed in terms of a Fourier series:

$$
f(x)=\sum_{n=0}^{\infty} A_{n} \sin (n \pi x)+B_{n} \cos (n \pi x) .
$$

Many oscillations of interest are relaxational, such as the firing of neurons and heart cells; a variety of models have been developed to describe relaxation oscillators [26, 27, 30]. Fig. 2.5 shows an example of an electrochemical relaxation oscillation. 


\subsection{Phase Models}

We want to understand and control the emergent behavior that arises from coupling between oscillating elements. In a chemical or biological system, it can be cumbersome to generate and work with a complete model of all species. Such a model can also be unintuitive and may be difficult to adapt for system changes. Additionally, it can be difficult to see commonalities between different phenomenological models (for example, in previous work this lab has shown that neurons and electrochemical oscillators synchronize in similar ways [31, 32]). Instead of modeling the underlying chemistry, we choose to model the observable behavior.

Any oscillatory signal can be assigned an instantaneous phase, $\phi_{j}(t)$. According to the Kuramoto phase model [8], a population of weakly-coupled oscillators can be written as

$$
\dot{\phi}_{j}(t)=\omega_{j}+\frac{K}{N} \sum_{n=1}^{N} H\left(\phi_{n}(t-\tau)-\phi_{j}(t)\right) \quad \text { for } j=1,2, \ldots N
$$

where $\omega_{j}$ is the natural oscillator frequency of oscillator $j, K$ is the coupling strength, $N$ is the number of elements, $H(\Delta \phi)$ is the coupling function, $\phi$ the phase, and $\tau$ is the time delay. The coupling function for perfectly smooth oscillators is $H(\Delta \phi)=\sin (\Delta \phi)$; in real systems there are usually additional harmonics. The coupling function is obtained by [33]

$$
H_{j}(\Delta \phi)=\frac{-2 \pi}{K P_{j}^{2}}\left[P_{j}(\Delta \phi)-P_{j}\right]
$$

where $P_{j}$ is the period of the uncoupled oscillator $\left(\omega_{j}=2 \pi / P_{j}\right)$, and $P_{j}(\Delta \phi)$ is the change in the period as a function of phase difference. Here, $\Delta \phi=\phi_{n}(t-\tau)-\phi_{j}(t)$. 



Figure 2.6: (Left) We perturb one of two identical systems with starting phase, $\phi$. The phase difference between the two systems due to the perturbation, $\Delta \phi^{*}$, gives the value of the response function, $Z(\phi)$, at the initial phase, $\phi$. (Right) Phase-advance and phase-lag due to perturbation as a function of oscillator phase, giving the response function, $Z$, in Eq. (2.6) [35].

The coupling function can also be written as [8]

$$
H(\Delta \phi)=\frac{1}{2 \pi} \int_{0}^{2 \pi} Z(\phi) h(\phi+\Delta \phi) d \phi,
$$

where $Z(\phi)$ is the response function and $h$ is the voltage perturbation $h(x(t))$ from Eq. (3.4) with $x(t)$ expressed as the phase. The response function gives the phase offset due to a differential perturbation over the oscillation; in some regions of the oscillation a perturbation will advance the resultant phase, while perturbations in other regions may result in a delay. Fig. 2.6 shows two systems, one which is not perturbed (orange square) and one which is perturbed. The response function is an inherent oscillator property that changes only with changes in system parameters such as the voltage, the acid concentration, or the circuit resistance. Detailed examples of these calculations can be found in Ref. [34]. 


\subsection{Time series analysis}

In order to implement the theory discussed in Section 2.2, we must be able to assign phases and radii to the time series.

\subsubsection{Peak and phase finding}

Throughout this dissertation, we use the algorithm described in this section for finding peaks. In the following we also define a method for finding phases; in Chapter 4, we introduce an instantaneous phase finder. Unless otherwise noted, we find phase via the peak-to-peak interpolation described here.

Ref. [35] describes in depth the methods for finding peaks and phase. We recap these methods here briefly. The Matlab code for both the peak and phase finder are in Appendix A.1.

First we find the peaks of the time series. We set a peak threshold, such that all the peaks of the data series will easily exceed this value. This creates a set of data windows where the time series exceeds the threshold. A window will only be considered if it contains a large enough set of points. This is to avoid spurious windows due to noise or other effects. Each window $j$ is fitted with a fourth-order polynomial. The location of the peak of the polynomial within the window closest to the largest value is taken to be the location of the peak within that window. This process is repeated for all windows, giving a vector of peak times.

Once the peaks are found, we can find the phase and the period. As shown in Fig. 2.7, each peak is defined as phase zero, and we define phase as linearly increasing between each peak. For example, the point which occurs halfway between a peak and the next peak is assigned the phase $\pi$ radians. The periods are just $t\left(\right.$ peak $\left._{j+1}\right)-t\left(\right.$ peak $\left._{j}\right)$. 


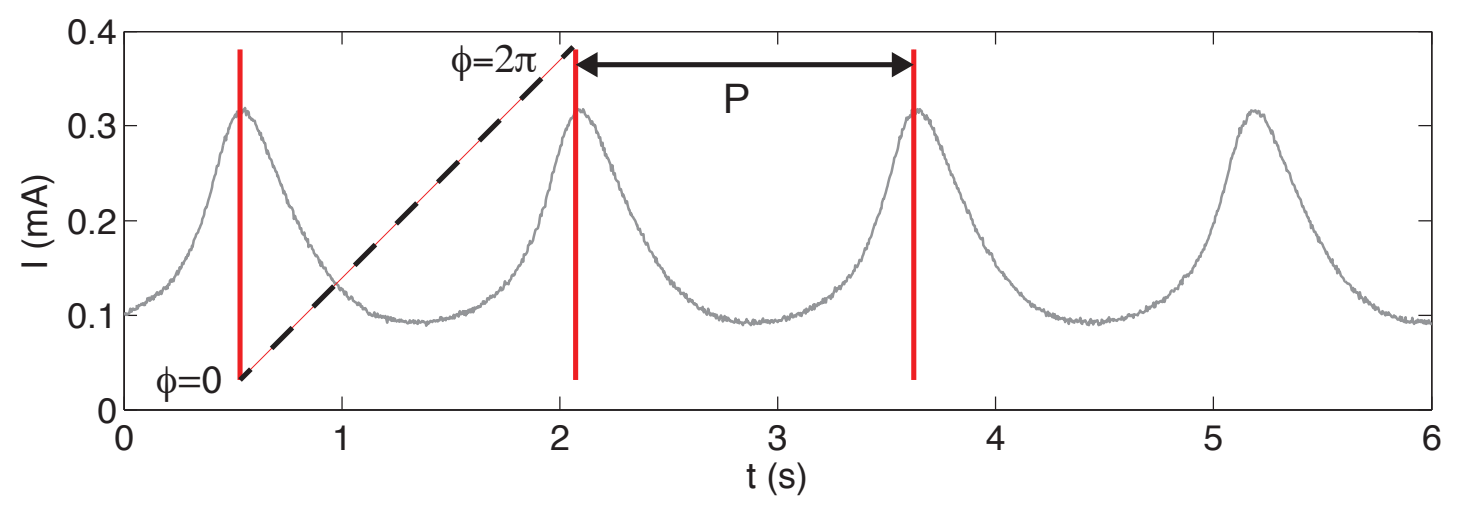

Figure 2.7: Finding phase from peak-to-peak linear interpolation. Peak times are shown by vertical red lines; in between peaks phase is linearly interpolated. Periods are found by the time between peaks.

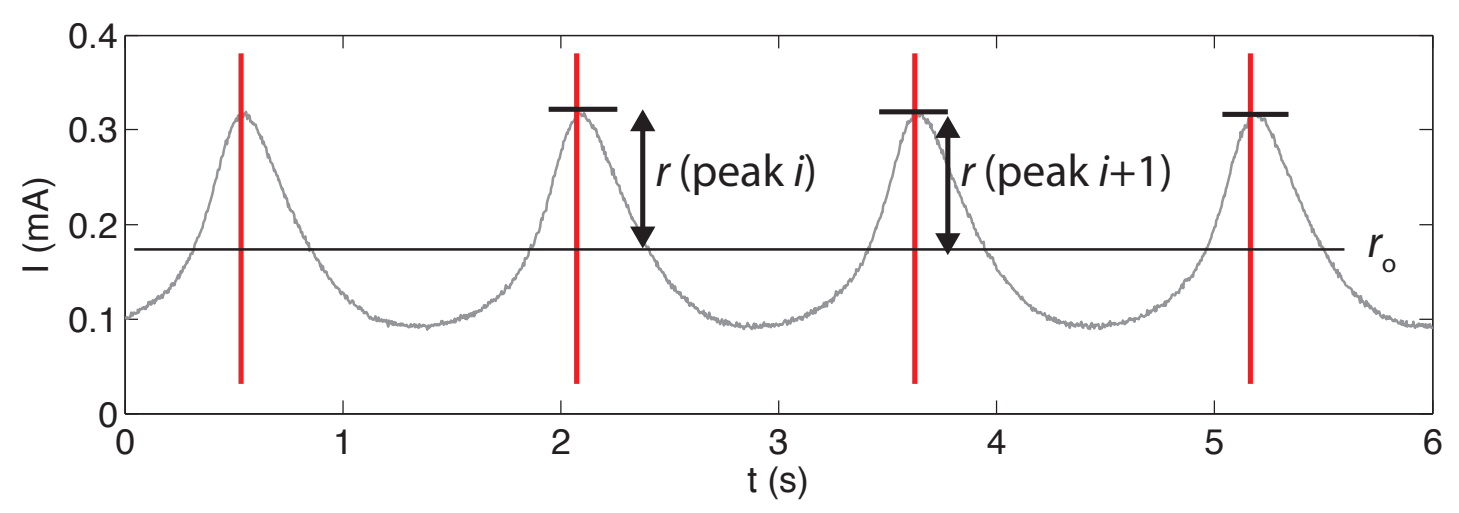

Figure 2.8: Peak times are shown by vertical red lines; the mean signal is shown by to horizontal black line. Radii $r_{j}$ are found for each peak $j$ as the difference between the mean and the value at the peak.

\subsubsection{Finding the the radius}

To find the radius, we first find the value of the times series at the time of each peak. This is subject to noise, and we therefore filter the initial time series with a fourth-order, 129 point Savitsky-Golay filter (SG filter). This filter is designed to preserve the form of the oscillation. The SG filter tends to underestimate sharp excursions, such as the peak, but preserves changes caused by coupling.

We define the radius as the value at the peak minus the mean of the oscillation. 
We use a slightly different definition, (peak-trough)/2 in Chapter 5. Peak-mean is generally preferable, because the trough can be become harder to fit as oscillations become more relaxational (see Fig. 2.5).

\subsection{Network Theory}

Any population of connected elements can be thought of as a network. Network theory has been used to study food webs [36], the internet [37], the power grid [38], epidemic spreading [39] and other systems. Elements of the network may be time-dependent, such as a neuron, or be static, such as a website on the internet. Connections between elements may be time-dependent, nonlinear, or time-delayed. Networks may have millions of elements. Due to all of these factors, the study of networks is a broad field of its own [40-42].

We use the connectivity matrix, $G$, to represent interactions between pairs of elements; $g_{j, n}$ is the pairwise coupling strength that element $j$ feels from element $n$. Fig. 2.9 shows a simple network in which oscillator 1 feels coupling from oscillator 2 with strength $x$, and oscillator 2 feels coupling from oscillator 3 with strength $2 x$. Fig. 2.9A shows a simple schematic while Fig. 2.9B shows the equivalent connectivity matrix, G.

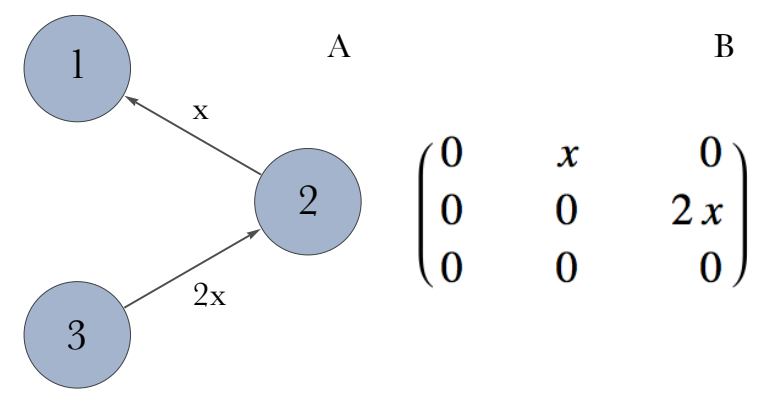

Figure 2.9: (A) A simple network, given in the text. (B) Connectivity matrix $G$ of simple network in (A). 
The Kuramoto phase model can be written for networks:

$$
\dot{\phi}_{j}(t)=\omega_{j}+K \sum_{n=1}^{N} g_{j, n} H\left(\phi_{n}(t-\tau)-\phi_{j}(t)\right),
$$

with $g_{j, n}$ now included in the model to indicate tunable pairwise interactions.

Many metrics have been used to numerically characterize networks. These metrics can make predictions about behavior on the network [43], and reveal non-obvious similarities or differences between networks. Suppose we have two populations of six elements; one of these populations is coupled globally (all $g_{j \neq n}=\frac{1}{N-1}$, and the other is coupled in a simple unidirectional ring $\left(g_{j=n+1}=1\right.$ and $g_{j \neq n+1}=0$ ), shown respectively in Fig. 2.10A and Fig. 2.10B.
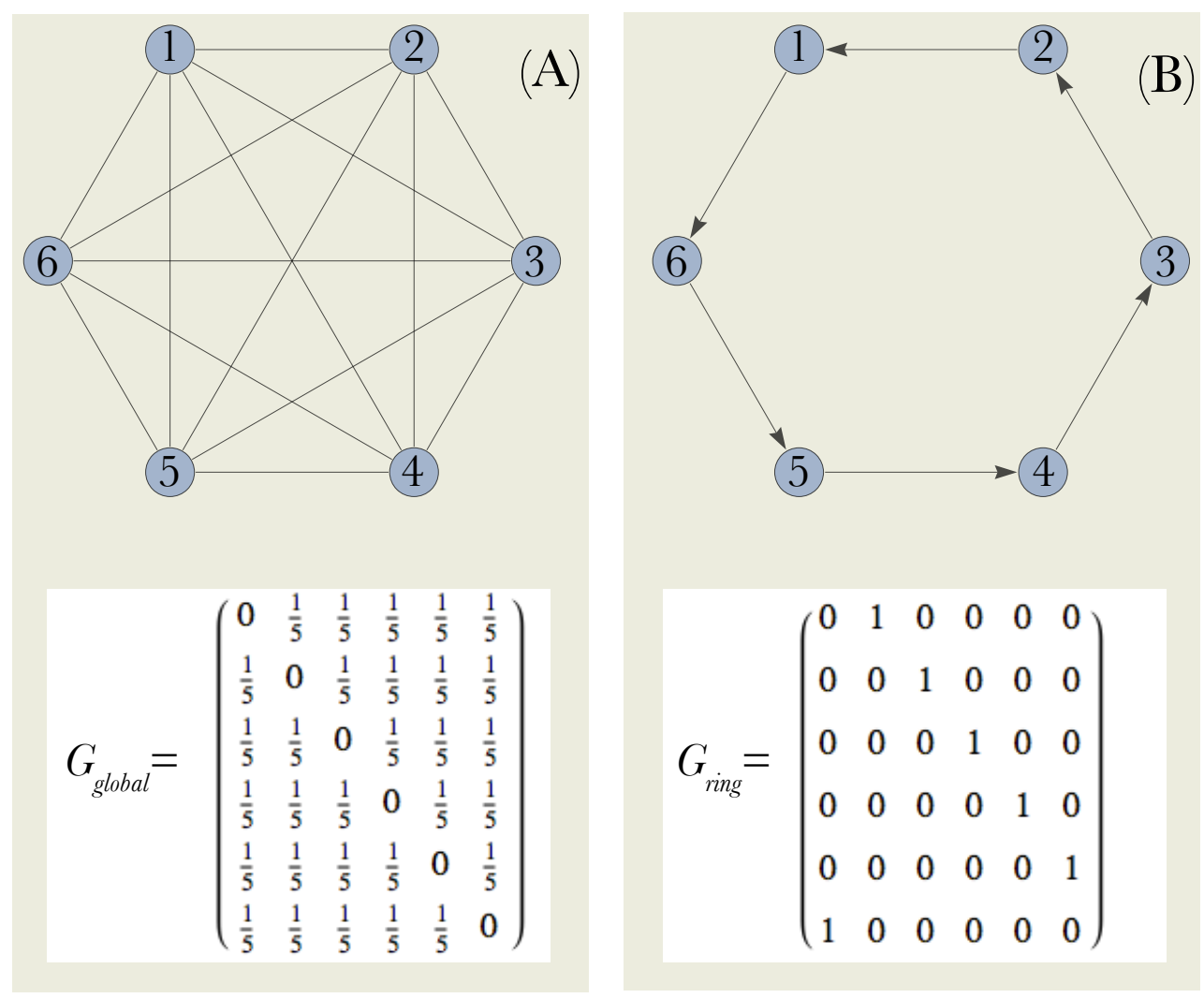

Figure 2.10: (A) Global coupling of 6 elements. (B) Unidirectional coupling of 6 elements. Top: simple schematic, bottom: connectivity matrix, $G$. 
We can use the diameter and the spectrum to illustrate the difference between global coupling and a simple network. We define a matrix $D$ as the shortest distance between all pairwise elements, such that $d_{j, n}$ is the smallest number of steps needed to get from element $n$ to element $j$. The matrix $D$ helps quantify how long it takes for information from one element to reach another element. The largest value of $D$ is called the diameter. The diameter of the global graph and unidirectional ring are 1 and 5 respectively. Information takes longer to traverse the ring, which will affect synchrony.

The spectra, and the quantities derived from it, are used in many network analytics [41]. To obtain the graph spectra, we first define the adjacency matrix, A. $A$ is a simplification of the graph $G$ in which $a_{j, n}=1$ if $g_{j, n} \neq 0$ and $a_{j, n}=0$ if $g_{j, n}=0$. The spectrum of $G$ is given by the eigenvalues of $A, \lambda$, where an eigenvalue is a number such that $\operatorname{det}(A-\lambda \mathbf{I})=0$. (Note that definitions vary in the literature; some define the spectrum as the eigenvalues of the Laplacian matrix, a zero rowsum version of the adjacency matrix, while some treatments require symmetrical coupling, that is, $g_{j, n}=g_{n, j}$, in $G[44,45]$.) One of the eigenvalues of $A$ in a constant row sum matrix will be the row sum; we are concerned with the other values. For global coupling, all non-row sum eigenvalues $\lambda$ are -1 . There are five uniquely valued non-row sum eigenvalues in the unidirectional ring: $\lambda= \pm \frac{1}{2} \pm \frac{\sqrt{3}}{2} i$ and $\lambda=-1$. The eigenvalue distribution gives information about the number of time scales in the network [46]; global coupling has only one while ring coupling has many. All time scales must be satisfied to achieve synchrony; this is an underpinning of the Master Stability formalism [47].

Both of the graphs presented in Fig. 2.10 are simple, but we can see with the two metrics presented that they will exhibit different synchronization behavior. We can guess, without any simulations, that the synchrony problem will be more complex 
for the unidirectional ring due to its large diameter and multivalued spectrum. It is interesting to note that there are 30 unidirectional connections in global coupling, while there are only 6 in the unidirectional ring. The ring coupling is more complex, although it has the same elements and fewer connections.

\subsubsection{The Master Stability Function}

The Master Stability Function (MSF) is a transform to analytically find the stability of system of elements connected by a network, G. In essence, the MSF says that a system will be stable if a certain value is less than zero for all the time scales of the system. It was originally developed to determine the stability of the synchronous state with no time delay $[47,48]$. It was recently extended to analyze clusters in network-coupled oscillators, as well as for time delay [49].

Here we lay out the original MSF derivation. Consider the system:

$$
\frac{d \mathbf{x}_{j}}{d t}=\mathbf{F}\left(\mathbf{x}_{j}\right)+\sigma \sum_{n=1}^{N} g_{j, n} \mathbf{H}\left(\mathbf{x}_{n}\right) .
$$

Where $\sigma$ is the coupling strength, $\mathbf{F}$ is the uncoupled behavior of the system, and $\mathbf{H}$ is a function of the dynamical variables for each oscillator that describes how the components of the system interact through coupling. We perform a 1st order Taylor series expansion, $\mathbf{x}_{n}(t)=\mathbf{s}(t)+\xi_{n}$, where $\xi$ is a small perturbation and $\mathbf{s}$ is a solution to the uncoupled system. When we expand all functions, this gives the variational equation, which shows how perturbations evolve along a trajectory,

$$
\frac{d \xi_{j}}{d t}=\sum_{n=1}^{N}\left[D \mathbf{F}(\mathbf{s}) \delta_{j, n}+\sigma g_{j, n} D \mathbf{H}(\mathbf{s})\right] \cdot \xi_{n},
$$

where $D \mathbf{F}$ and $D \mathbf{H}$ are the Jacobians of the functions $\mathbf{F}$ and $\mathbf{H}$, and $\delta_{j, n}$ is the 




Figure 2.11: The Master Stability Function for the Rössler oscillators with different coupling and parameters. (Figure 1 from [51].)

Kronecker delta function. This equation can be rewritten to give:

$$
\frac{d \zeta_{l}}{d t}=\left[D \mathbf{F}(\mathbf{s})+\sigma \gamma_{l} D \mathbf{H}(\mathbf{s})\right] \cdot \zeta_{l},
$$

where $\gamma_{l}$ is an eigenvalue of the coupling matrix. From this equation, we can solve for the Floquet exponents, $\mu$, in the case of a limit cycle oscillator, or the Lyapunov exponents, $\lambda$, in the case of a chaotic oscillator. The MSF is $\mu_{\max }$ or $\lambda_{\max }$ as a function of $\alpha$, where $\alpha=\sigma \gamma[47,48,50]$.

Fig. 2.11 shows the MSF for the Rössler oscillator under chaotic and limit cycle conditions, with $\mathrm{x}$ - and $\mathrm{y}$-component coupling; when $\lambda_{\max }<0$, the synchronous state will be stable. All types of coupling in the Rössler oscillator synchronize first at a lower gain, and then desynchronize at a higher gain. We note that this is an interesting behavior; the phase model can never desynchronize due to increasing gain. The MSF of the Rössler oscillator shows that synchrony can be disrupted 
by sufficiently strong coupling, or if a coupling network has a sufficiently broad spectrum. Given an MSF, one can predict if it is possible to synchronize a specific network. For example, $N=34$ is the maximum number of $x$-coupled chaotic Rössler oscillators that can be synchronized with any coupling strength in a star network (one hub and $N-1$ spokes) [48]. Mathematics for the extension of the model for time-delay and cluster states can be found in Ref. [49] and in our paper in Chapter 5. 


\section{Chapter 3}

\section{Experimental Apparatus}

\subsection{Introduction}

We used an oscillatory nickel-oxide experimental system to study the dynamics of oscillatory populations. We performed simulations using various models.

\subsection{Electrochemical system}

Our nickel oxide system is a good proxy for the broader study of interacting oscillators; it has low noise, high controllability, and oscillates on a convenient timescale that is fast enough to allow the recording of many oscillations, but slow enough so that the data can be readily sampled. By tuning various parameters, we can choose the oscillation type, coupling type, and form of coupling. 


\subsubsection{Experimental System}

The following system of reactions describes electrochemical dissolution of nickel in sulfuric acid [52]:

$$
\begin{aligned}
\mathrm{Ni} & \rightarrow \mathrm{Ni}^{2+}+2 e^{-} \\
\mathrm{Ni}+\mathrm{H}_{2} \mathrm{SO}_{4}^{-} & \rightarrow \mathrm{NiH}_{2} \mathrm{SO}_{4}^{-} \\
\mathrm{H}_{2} \mathrm{O} & \rightarrow 2 \mathrm{H}^{+}+\frac{1}{2} \mathrm{O}_{2}+2 e^{-} \\
\mathrm{Ni}+\mathrm{H}_{2} \mathrm{O} & \rightarrow \mathrm{NiOH}+\mathrm{H}^{+}+e^{-} \\
\mathrm{NiOH} & \rightarrow \mathrm{NiO}+\mathrm{H}^{+}+e^{-} \\
\mathrm{NiO}+2 \mathrm{H}^{+} & \rightarrow \mathrm{Ni}^{2+}+\mathrm{H}_{2} \mathrm{O} .
\end{aligned}
$$

This system has an open-circuit voltage of $-0.68 \mathrm{~V}$ vs. the $\mathrm{Hg} / \mathrm{Hg}_{2} \mathrm{SO}_{4} / \mathrm{K}_{2} \mathrm{SO}_{4}$ (sat) reference electrode.

The oscillatory form can be tuned by the applied potential; potentials near the Hopf bifurcation will be smooth with fewer significant harmonics (see Fig. 3.1B) while potentials farther from the Hopf bifurcation will be relaxational with more significant harmonics (see Fig. 3.1C). Oscillations do not occur above about 1.25V (this number can vary slightly depending upon the potentiostat); Fig. 3.1A shows the bifurcation diagram of the nickel oxide system. Fig. 3.1B shows the waveform of a smooth oscillator at $V=1.105 \mathrm{~V}$; Fig. 3.1C shows the waveform of a relaxational oscillator at $V=1.225 \mathrm{~V}$.

Between $V \approx 1.05 \mathrm{~V}$ and $V \approx 1.23 \mathrm{~V}$, when a nickel oxide layer is present, localized negative differential resistance occurs, as well as bistability between a high current and a low current. These conditions occur due to competition by sulfate and bisulfate groups for surface sites. When an additional resistance is applied (we use 

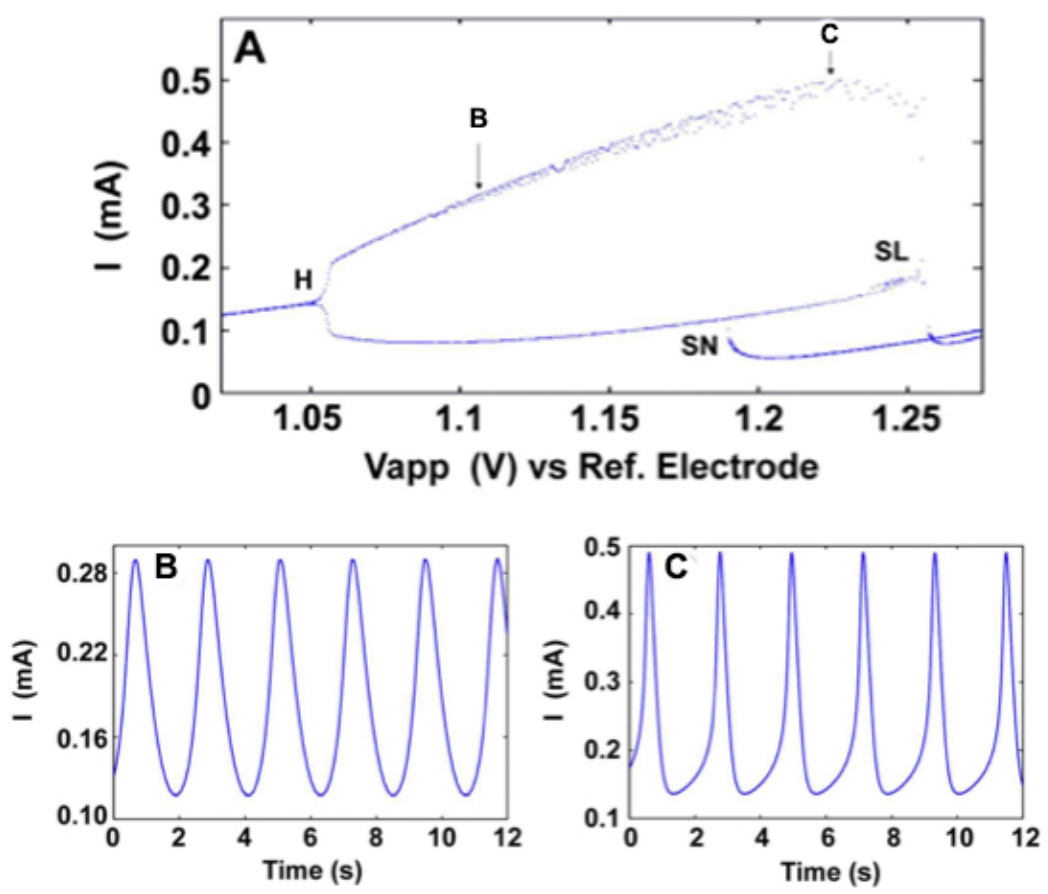

Figure 3.1: (A) Bifurcation diagram for nickel electrodissolution when $\left|\mathrm{H}_{2} \mathrm{SO}_{4}\right|=3.0 \mathrm{M}$ and $\mathrm{R}=650 \Omega$. The bifurcations are labeled: Hopf $(\mathrm{H})$, Saddle Node (SN) and Saddle Loop (SL) $[35,52,53]$. (B) Time series of smooth oscillations at $V=1.105 \mathrm{~V}$. (C) Time series of relaxational oscillations at $V=1.225 \mathrm{~V}$ [35].

$R_{p}=650 \Omega$ ) in the voltage range of negative differential resistance, this causes the eigenvalues to cross the imaginary axis, resulting in a Hopf bifurcation [18, 52].

\subsubsection{Electrode preparation}

The procedures outlined in this section are very similar to those described in Ref. [35], Section 3.2.2.

We assemble the electrode array from $99.98 \%$ pure nickel wire with a diameter of $1 \mathrm{~mm}$. We seat these wires in epoxy, which spaces them and provides electrical insulation. In this way, reactions can only occur at the ends of the electrodes. The detailed procedure for building the electrode array is given below, with accompanying 
images in Fig. 3.2.

1. Cut 64 pieces of nickel wire to a length of about $10 \mathrm{~cm}$; roll to ensure straightness.

2. Prepare the $1 / 8$ " teflon spacers that will hold the top and bottoms of the wires:

(a) Drill 1-mm holes for the 64 wires in the desired pattern.

(b) Cut divots into one of the teflon disks.

3. Solder an 8 " teflon coated wire to each of the 64 nickel wires.

(a) Cover these 64 solders with a rubber shrink tube.

4. Thread the 64 wires through the holes in the teflon disks. The disk without divots should be at the bottom of the nickel portion of the wire while the disk with divots should be at the top.

5. Above the divoted teflon disk, place 64 rubber shrink tube on the teflon portion of the wire. Do not shrink them.

6. Solder the teflon wires to three 25 pin male D-sub connectors ( 25 to the first, 25 to the second, and 14 to the third).

(a) Slide the shrink tubes over these connections and shrink them.

7. Check the electrical contact of all wires. Ensure that all the wires are electrically isolated.

8. Place assembly in a 2" diameter teflon mold.

9. Mix the epoxy resin (1 part epoxy (Type W-7), 1 part hardener (Type A)) and pour slowly into the mold to avoid the creation of air pockets. Allow 24-48 hours for the resin to harden. 


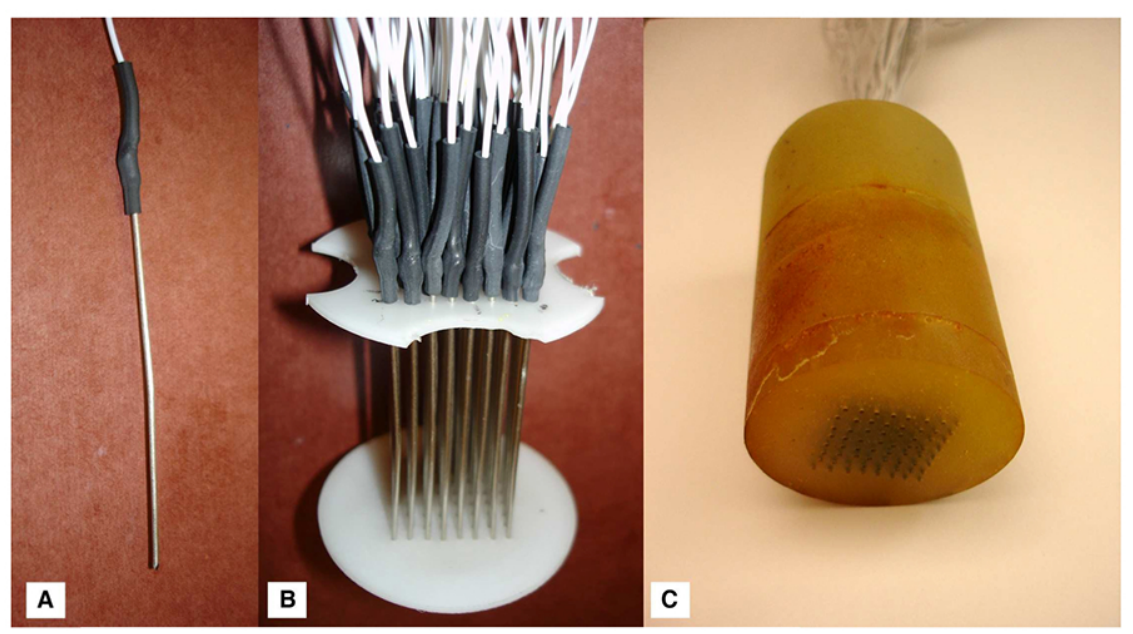

Figure 3.2: The fabrication of the 64 electrode array. (A) An individual nickel wire soldered to a teflon coated wire. (B) 64 wires arranged with the teflon spacers. (C) The fully assembled array [35].

\subsubsection{Experimental procedure}

We perform experiments using up to $641-\mathrm{mm}$ diameter Ni working electrodes (99.98\% pure) embedded in resin so that reactions occur only at the wire end. The electrochemical cell consists of the electrodes in a $3 \mathrm{M} \mathrm{H}_{2} \mathrm{SO}_{4}$ electrolyte, a Pt mesh counter electrode, and a $\mathrm{Hg} / \mathrm{Hg}_{2} \mathrm{SO}_{4} / \mathrm{K}_{2} \mathrm{SO}_{4}$ (sat) reference electrode. A jacketed glass vessel enclosed the cell and maintained a temperature of $11^{\circ} \mathrm{C}$. A potentiostat set the electrode potential $V$ of the electrodes such that they undergo transpassive dissolution, where the nickel may tunnel through the nickel oxide layer present during the experiment. We can set electrode potential $V_{j}$ and voltage perturbations $\delta V_{j}$ individually with the ACM Instruments multi-channel potentiostat. The apparatus is shown in Figs. 3.3 and 3.4.

Before each experiment, we polish the electrodes with a wet grinder, first with a rough grit (180 grit) and then with increasingly smoother grits (up to 4000 grit). The polishing removes the oxide layer from the previous experiment and provides a consistent starting condition. 



Figure 3.3: (Left) Experimental apparatus with ACM Instruments 64channel potentiostat. (Right) Photo of the physical electrochemical cell.

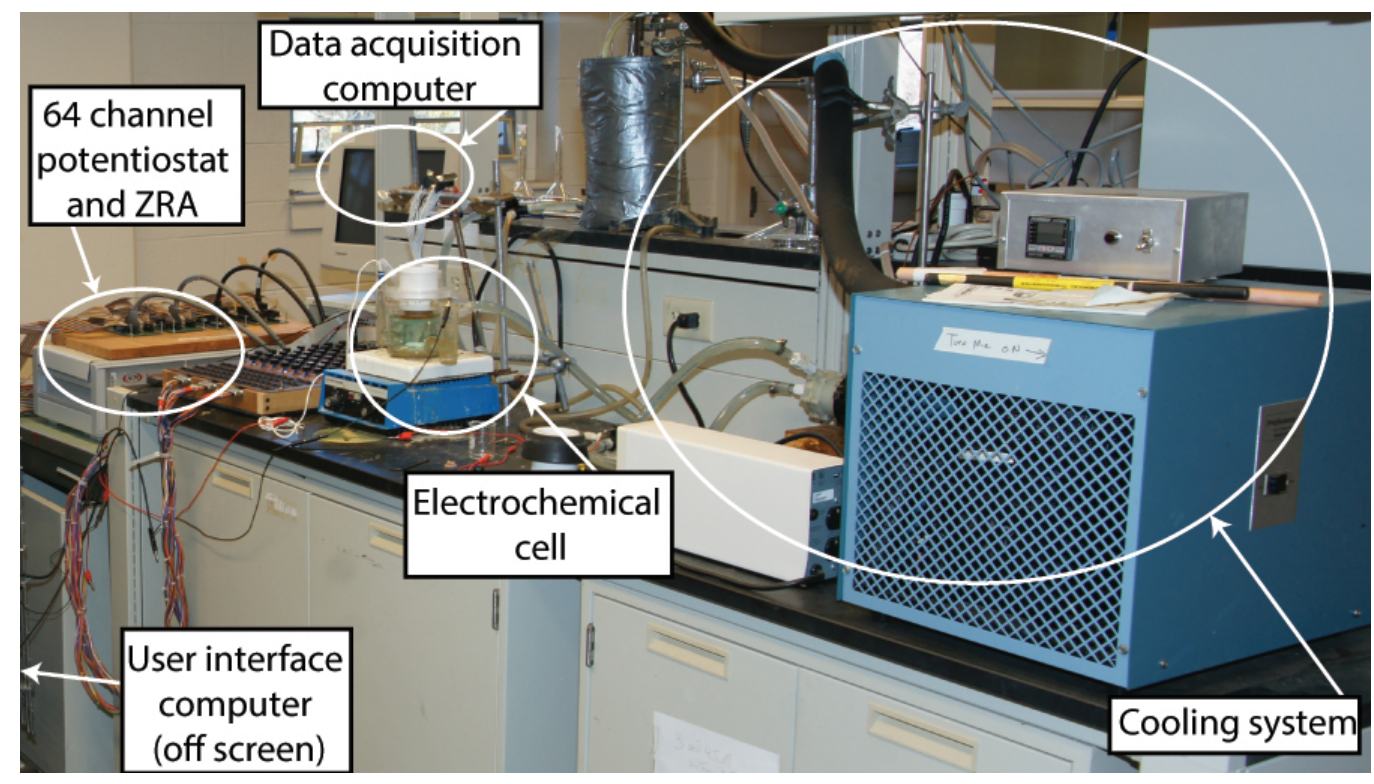

Figure 3.4: Labeled photo of the entire experimental apparatus.

We connect the cell to the potentiostat and ZRA, and ramp from $-0.68 \mathrm{~V}$ to $1.25 \mathrm{~V}$ and back down to $0 \mathrm{~V}$ at a rate of $10 \mathrm{mV} / \mathrm{s}$. The purpose of this voltage sweep is to form the nickel oxide layer on the surface of the electrodes. Oscillations are due to the formation and dissolution of this layer. After the initialization, we let the oscillators rest for about two hours. The oscillator frequency drifts quickly following initialization; this drift never stops, but it slows over time, and is relatively 
constant after two hours.

We physically couple the oscillators via perturbations to the applied potential. Two computers and several Labview programs calculate and apply these perturbations (see Ref. [35] for more description of these programs). A data acquisition computer measures the electrode currents at $250 \mathrm{~Hz}$. A Labview based program on the host computer then calculates the voltage perturbations.

First, we scale the current:

$$
\bar{I}_{j}(t)=\frac{\left\langle A_{a l l}\right\rangle}{A_{j}}\left(I_{j}(t)-\left\langle I_{j}\right\rangle\right)
$$

where $\left\langle I_{j}\right\rangle$ is the mean value of the channel, $A_{j}$ is the amplitude of oscillator $j$, and $\left\langle A_{\text {all }}\right\rangle$ is the mean amplitude of the population. Then we calculate the potential drop across the double layer:

$$
x_{j}(t)=V_{j}(t)-\bar{I}_{j}(t) R_{p}
$$

where $V_{j}(t)$ is the applied voltage of channel $j$. Finally, we calculate the voltage perturbations, $\delta V_{j}$ :

$$
\begin{gathered}
h\left(x_{j}(t)\right)=\sum_{s=0}^{S} k_{s} x_{j}\left(t-\tau_{s}\right)^{s} \\
\delta V_{j}=\sum_{n=1}^{N} g_{j, n} h\left(x_{n}(t)\right)
\end{gathered}
$$

where $S$ is the harmonic of the feedback, $k_{s}$ is the feedback magnitude of the $S=s$ harmonic, and $\tau_{s}$ is the delay of the $S=s$ harmonic. $g_{j, n}$ are elements of the coupling matrix, or graph, that gives the pairwise interaction strengths between all oscillators. We often normalize the coupling matrix, $G$, such that all row sums are unity. The coupling matrix required for Labview must be tab-delimited in a specific format; 
see Appendix A.4 for code to output such a matrix.

\subsection{Simulation and analysis methods}

We simulated many of our experiments with ordinary and delayed differential equations. We used a fourth-order Runge-Kutta to compute all ODEs and DDEs (see code in Appendix A.5).

Per Eq (2.5), we find $H(\Delta \phi)$, the period as a function of the phase difference, with one data point per oscillation. For the purposes of modeling and simulation, we want a direct relationship between the two. We achieve this by performing a Fourier fit of $H$. Fourier fits require evenly-sampled data; the initial data is often highly non-uniform in its sampling, so we resample using a sliding window averager. We typically keep the first 5-7 coefficients of the Fourier series. Appendix A.2 gives the relevant code. 


\section{Chapter 4}

\section{Reconstruction of two-dimensional phase dynamics ${ }^{1}$}

\subsection{Notes to the reader}

This chapter closely mirrors our 2011 paper with Michael Rosenblum and Arkady Pikovsky [54]. Here, we select pairs of dissimilar oscillators (achieved via different applied voltages) from the whole population of 64 . We couple these pairs with weak coupling that does not induce synchrony. For Figure 4.9, we drive a single smooth oscillator with a sine wave. Please note that Section 4.4 describing the experimental apparatus is mostly information repeated from Chapter 3. We repeat this information for the convenience of the reader. Also note that Section 4.7, the discussion of this chapter, has been re-written for greater readability.

\footnotetext{
${ }^{1}$ K. A. Blaha, A. Pikovsky, M. Rosenblum, M. T. Clark, C. G. Rusin and J. L. Hudson, "Reconstruction of two-dimensional phase dynamics from experiments on coupled oscillators" Phys. Rev. E, 84, $046201,2011$.
} 


\subsection{Introduction}

Scientists have investigated systems of coupled oscillators in a variety of fields. Examples include coupled lasers [1], population dynamics [2], chemical reactions [3], and cardiorespiratory interactions $[5,6]$, among others. There are two ways to obtain a theoretical description of the system: either write the model equations for the coupled systems starting from the first principles, or reconstruct the model equations from observations. In many cases, e.g. in biological systems, use of the former approach is greatly impeded by underlying complexity and lack of knowledge about oscillation generation and coupling mechanisms.

This paper takes the second approach and reconstructs the interaction between a pair of experimental nonlinear electrochemical oscillators. We discuss the basic theory, which we apply for our system of two oscillators with weak coupling. The system is represented in terms of two phases, which in many cases may be simplified to a single variable, the phase difference [8]. We show that phase models that preserve dependence on individual phases generally provide a more detailed description of the interactions between two oscillators than do those based on the phase difference. We compare results of the two modeling methods and discuss limitations of models based on phase difference. We calculate from experimental data a two-phase model using a previously introduced technique [55]. Our experimental results verify phase reconstruction in a system with noise and connect the two-dimensional and one-dimensional models $[8,56,57]$. We calculate the natural oscillator frequencies, changes in coupling directionality and coupling time delay from the experimentally determined phase models. We also present experiments where coupling functions exhibit higher order terms, and show that the one-dimensional model does not capture these terms. 


\subsection{Theory}

Suppose we observe two interacting oscillators described by

$$
\dot{\vec{x}}_{j}=\vec{F}_{j}\left(\vec{x}_{j}\right)+\varepsilon \vec{p}_{j}\left(\vec{x}_{j}, \vec{x}_{n}\right),
$$

where $j=1,2, n=2,1$, and the parameter $\varepsilon$ describes the strength of the interaction. Generally, the functions $\vec{F}_{i}$ are different; moreover they can be of different dimension. The coupling functions $\vec{p}_{i}$ can be different as well. We assume that both systems when uncoupled, i.e. when $\varepsilon=0$, possess stable limit cycles in their phase spaces. The asymptotic dynamics of each oscillator (after transients die out) can be then described by a single variable, the phase $[7,8,56]$.

Even when the Eqs. (4.1) of the coupled oscillating system are known their analytical treatment can be quite complicated. A simplification can be made in the case of weak coupling, where applied perturbations are small compared to the negative Lyapunov exponent(s) of each oscillator. For this case the oscillators remain near their closed orbits and the dynamics of a pair of coupled systems is confined to the two-dimensional torus in the phase space. Correspondingly, the dynamics can be parameterized by two phases $[7,8,56]$,

$$
\dot{\phi}_{j}=\omega_{j}+Q^{(j)}\left(\phi_{j}, \phi_{n}\right)
$$

Here $\phi_{j}$ is the phase of oscillator $j, \phi_{n}$ is the phase of the other oscillator and $\omega_{j}$ is the natural angular frequency of the oscillator $j$, i.e. the frequency of the uncoupled system. The functions $Q^{(j)}$ describe the coupling between the systems. The only $a$ priori assumption about these functions is that they are $2 \pi$-periodic with respect to both arguments; in particular, they can contain a constant term. 
If the dynamical Eqs. (4.1) are known, the coupling functions $Q^{(j)}$ can be represented in the form of the power series by means of a perturbative expansion [8],

$$
Q^{(j)}\left(\phi_{j}, \phi_{n}\right)=\varepsilon Q_{1}^{(j)}\left(\phi_{j}, \phi_{n}\right)+\varepsilon^{2} Q_{2}^{(j)}\left(\phi_{j}, \phi_{n}\right)+\ldots
$$

where the subscripts on $Q^{(j)}$ correspond to the order of approximation. Computation of the high-order terms represents, to the best of our knowledge, an unsolved problem, whereas the first-order phase approximation is widely used in various applications $[8,57]$. The first-order coupling functions can be written as

$$
Q_{1}^{(j)}\left(\phi_{j}, \phi_{n}\right)=\vec{Z}_{j}\left(\phi_{j}\right) \cdot \vec{h}_{j}\left(\phi_{j}, \phi_{n}\right),
$$

where $\vec{Z}$ is the phase-dependent response function of the oscillator and $\vec{h}=\vec{p}\left(\vec{x}_{j}\left(\phi_{j}\right), \vec{x}_{n}\left(\phi_{n}\right)\right)$ is the applied stimulation. In the simplest case when the scalar driving is independent of the phase of the driven system and enters the state-space Eqs. (4.1) as an additive term, the coupling function can be represented as a product of two functions of one variable, $Q_{1}^{(j)}\left(\phi_{j}, \phi_{n}\right)=Z_{j}\left(\phi_{j}\right) h_{j}\left(\phi_{n}\right)$. The phase description Eq. (4.2) can be valid for not-so-weak coupling as well: as long as a stable invariant torus in the phase space exists, the motion on it can be parametrized by the phases and the dynamics can be written in the form of Eq. (4.2).

A large body of work concerns the description of two interacting oscillators as a function of the phase difference [11]. Theoretical studies and numerical simulations show that these one-dimensional phase models capture the important synchronization properties of populations of similar oscillators with weak interactions $[9,10,12,58-61]$. Phase difference based phase models also predict system behavior in electrochemical experiments [62]. The one-dimensional approach is suitable, because the long-term dynamical effects, such as synchronization, depend 
mainly on the averaged coupling functions $q$, discussed below.

The reduction to a one-dimensional description can be made if the coupling is also weak compared to the natural frequency, i.e. the norm of the coupling function $\left\|Q^{(j)}\right\| \ll \omega_{i}$, and the natural frequencies of two systems are close to a resonance condition, $m \omega_{1} \approx n \omega_{2}$. In this case one can introduce a slow variable, phase difference,

$$
\psi=n \phi_{2}-m \phi_{1}
$$

and average Eq. (4.2) over the common oscillation period $P=2 \pi n / \omega_{1} \approx 2 \pi \mathrm{m} / \omega_{2}$. In the case of similar oscillators $(m=n=1), \psi$ reduces to $\phi_{2}-\phi_{1}$. The averaged equations have the form [8],

$$
\dot{\phi}_{j}=\omega_{j}+q_{m, n}^{(j)}(\psi),
$$

where the new, averaged coupling function $q_{m, n}$ is a function of the phase difference only,

$$
q_{m, n}^{(j)}(\psi)=\frac{\varepsilon}{2 \pi} \int_{0}^{2 \pi} Q^{(j)}\left(\phi_{1}, \frac{m}{n} \phi_{1}+\frac{\psi}{n}\right) d \phi_{1} .
$$

Thus, a description in terms of phase difference is possible only in vicinities of the resonant frequency ratios. For each of the resonant tongues one should establish an averaged coupling function $q_{m, n}$. Thus, although a complete description of the coupled system for any frequency ratio can be achieved with one pair of twodimensional functions $Q$, a large set of one-dimensional coupling functions is required to provide the same result.

We illustrate the difference in synchronization predictions between two- and one-dimensional phase models by an analysis of the following toy model of a 
harmonically driven oscillator

$$
\begin{aligned}
& \dot{\phi}_{1}=\omega_{1}+\varepsilon\left[\cos \left(\phi_{1}\right)+\cos \left(2 \phi_{1}\right)\right] \sin \left(\phi_{2}\right) \\
& \dot{\phi}_{2}=\omega_{2} .
\end{aligned}
$$

Averaging Eq. (4.7) using Eq. (4.6) yields two nontrivial one-dimensional coupling functions $q_{1,1}=\frac{\varepsilon}{2} \sin \left(\phi_{2}-\phi_{1}\right)$ and $q_{2,1}=\frac{\varepsilon}{2} \sin \left(\phi_{2}-2 \phi_{1}\right)$; all other functions $q_{n, m}=0$. Thus, the averaged description of Eq. (4.7) predicts locking only when $\omega_{1} \approx \omega_{2}$ and $2 \omega_{1} \approx \omega_{2}$, with triangular Arnold tongues. However, the tongues
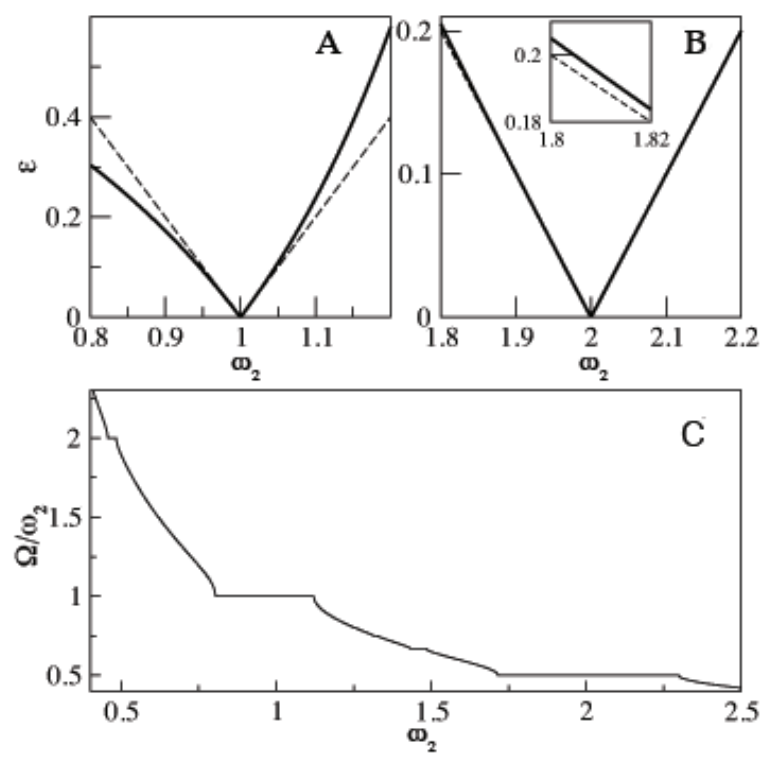

Figure 4.1: (a) Main synchronization tongue for the two-dimensional model Eq. (4.7) (solid line) and the tongue predicted by the one-dimensional model $q_{1,1}$ (dashed line). (b) The second tongue at $\omega_{2} \approx 2$ for the two-dimensional model (solid line) and for the one-dimensional model $q_{2,1}$ (dashed line). Here the averaging works well and the difference is pronounced only far from resonance, see inset. (c) Devil's staircase for the toy model Eq. (4.7) with $\omega_{1}=1$, for $\varepsilon=0.3$; here $\Omega=\langle\dot{\phi}\rangle$ where $\langle\cdot\rangle$ is time average. Locking regions at $\Omega / \omega_{2}=2, \Omega / \omega_{2}=1, \Omega / \omega_{2}=2 / 3$, and $\Omega / \omega_{2}=1 / 2$ are seen. Zoom of the plot (not shown) exhibits further locking ratios, e.g. 3/2, 4/5, 3/4, and $3 / 5$. 
obtained by numerical simulation of the full model (4.7) differ from the triangular shape when $\omega_{2}$ is farther from the resonance frequencies, as shown in Fig. $4.1 \mathrm{~A}$ and B. Furthermore, numerical analysis of Eq. (4.7) shows many locking regions, as seen in Fig. 4.1C, in contrast to prediction of the reduced model. We explore the applications of the two-dimensional method and compare the one- and two-dimensional methods in the following sections.

\subsection{Experimental setup}

Experiments were performed on an electrochemical cell consisting of two Ni working electrodes (99.98\% pure), a Pt mesh counter electrode, and $\mathrm{Hg} / \mathrm{Hg}_{2} \mathrm{SO}_{4} / \mathrm{K}_{2} \mathrm{SO}_{4}$ (sat) reference electrode, with a $3 \mathrm{M} \mathrm{H}_{2} \mathrm{SO}_{4}$ electrolyte, shown in Fig. 4.2A. The cell was enclosed in a jacketed glass vessel maintained at a temperature of $11^{\circ} \mathrm{C}$. An ACM Instruments multi-channel potentiostat was used to individually set the electrode potential $V_{i}$ of each electrode such that the electrodes undergo transpassive dissolution.

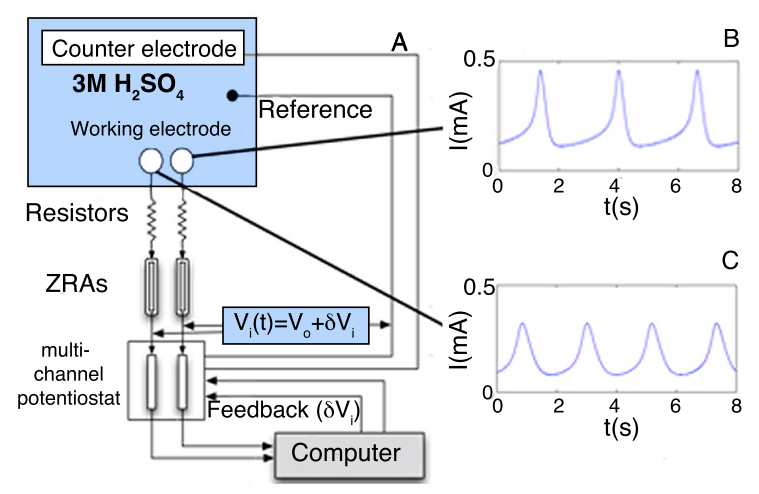

Figure 4.2: (a) Experimental apparatus with multi-channel addressable voltage and feedback. (b) Time series of relaxational oscillations at $V=1.210 \mathrm{~V}$. (c) Electrochemical dissolution time series showing smooth oscillations at a potential of $V=1.105 \mathrm{~V}$. 
A $650 \Omega$ resistor was attached to each electrode, causing the dissolution current to oscillate [52]. The resulting dissolution currents were measured using a zero resistance ammeter attached to a real time data acquisition system, Fig. 4.2A. The shape of the electrochemical oscillator waveform depends on applied voltage. Smooth oscillations with a natural frequency of about $0.5 \mathrm{~Hz}$ are observed at potentials of approximately $1.105 \mathrm{~V}$. Relaxational oscillations with a frequency of about $0.35 \mathrm{~Hz}$ are observed around $1.20 \mathrm{~V}$ [63]. As the applied voltage of each electrode in the experimental system can be chosen independently, any combination of smooth or relaxation oscillators is accessible. Relaxational and smooth oscillations are shown in Fig. 4.2B and Fig. 4.2C, respectively. Here, oscillator 1 refers to the more relaxational oscillator $\left(V_{1}=1.180 \mathrm{~V}\right)$ with a natural frequency of $v_{1}=0.405 \mathrm{~Hz}$ $\pm 0.005 \mathrm{~Hz}$, while oscillator $2\left(V_{2}=1.105 \mathrm{~V}\right)$ refers to the smooth oscillator with a natural frequency of $v_{2}=0.479 \mathrm{~Hz} \pm 0.002 \mathrm{~Hz}$, where $v=\omega / 2 \pi$. The range is due to the slow drift over time of the natural frequencies of the oscillators as an inherent property of the system.

Negligible intrinsic electrical interactions exist between the uncoupled oscillators. The startup or shutdown of an oscillator does not alter the behavior of the second oscillator. Furthermore, the oscillator dynamics have no interdependence when both oscillators are functioning in the uncoupled state.

Interactions were introduced using real-time coupling of the form:

$$
\begin{aligned}
& \Delta V_{1}(t)=K\left[k_{1} x_{2}(t-\tau)\right] \\
& \Delta V_{2}(t)=K\left[k_{2} x_{1}(t-\tau)\right]
\end{aligned}
$$

where $\Delta V_{1,2}$ are the changes in the circuit potentials of the elements, $K$ is the fixed overall coupling gain, $k_{1}$ and $k_{2}$ are the coupling gains on oscillator 1 and oscillator 
2 , respectively, such that $0 \leqslant k_{j} \leqslant 1$, and $\tau$ is the coupling time delay. The scaled potentials of the elements as a function of time $x_{j}(t)$ are,

$$
x_{j}(t)=V_{j}(t)-I_{j}(t) R_{p},
$$

where $V_{j}$ are the applied potentials, $I_{j}$ are the normalized currents, and $R_{p}=650 \Omega$ is the channel resistance. Only linear coupling is considered here, with and without time delay $[64,65]$.

\subsection{Methods}

The only information required by the phase models in Eqs. (4.2) and (4.5) is the instantaneous phases. The instantaneous phases are calculated directly from the electrochemical current time series using the phase space angle, as shown in Fig. 4.3. Other definitions of phase are equally applicable provided they yield a one-to-one correspondence between phase and location on the closed orbit $[15,57,66]$. For example, phase defined from percentage of trajectory length between consecutive Poincaré surface of section crossings is useful for more complicated oscillations (e.g. electrocardiograms) [55].

Electrodissolution currents of each element are measured at $250 \mathrm{~Hz}$, filtered with a 129 point fourth order Savitsky-Golay filter, and used to calculate the genuine phases. The Savitsky-Golay filter preserves the structure of the oscillation while removing nonphysical phase velocities caused by noise in the system. Although the maximum amplitude of the relaxation oscillator (oscillator 1) tends to be underestimated, the phase is well-preserved; the difference between the phases calculated from filtered and unfiltered signals is delta correlated.

Note that definitions of phase based on the Hilbert transform have inherent 

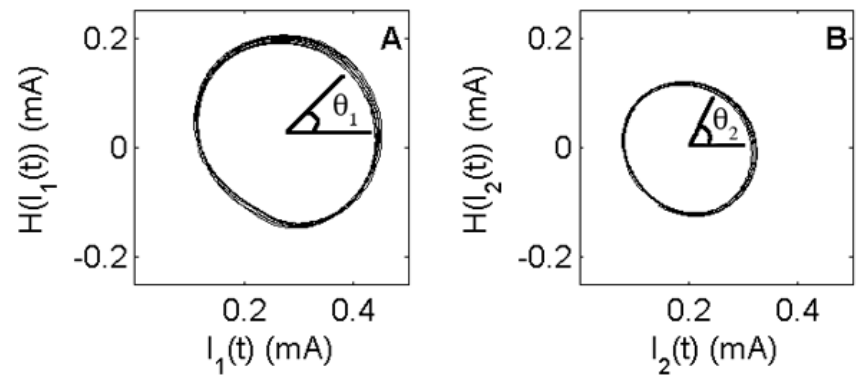

Figure 4.3: Time series in Hilbert space; $\theta_{i}$ indicates protophase (a) for the relaxational oscillator, (b) for the smooth oscillator. Note that although the phase portraits look very much alike, the distributions of $\theta_{i}$ are different, as shown in Fig. 4.4B.
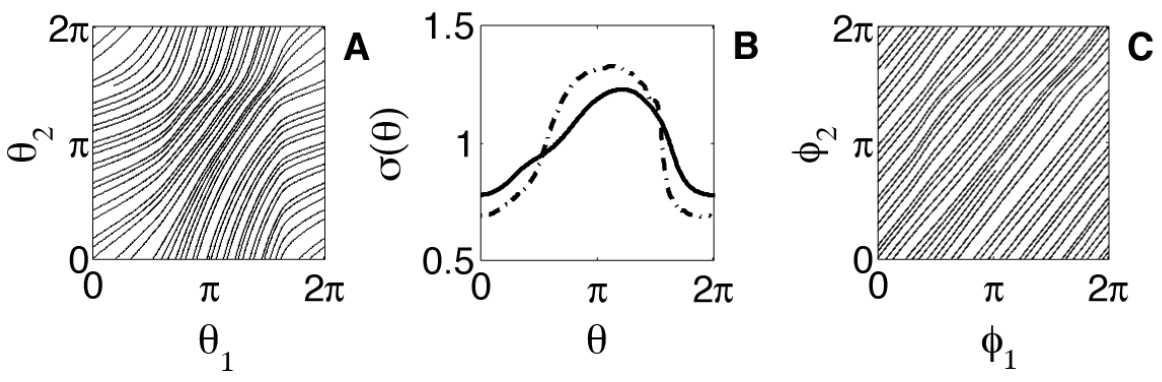

Figure 4.4: (a) Wrapped protophase of oscillator 1 (relaxational, $V_{1}=$ $1.180 \mathrm{~V}$ ) versus protophase of oscillator 2 (smooth, $V_{2}=1.105 \mathrm{~V}$ ) obtained via the Hilbert transform. (b) Phase transformation function, $\sigma . \sigma\left(\theta_{1}\right)$ is dash-dotted, and $\sigma\left(\theta_{2}\right)$ is solid. (c) Wrapped genuine phases of the two oscillators, $\phi_{1}$ and $\phi_{2}$.

deviations in phase velocity as a function of phase. These deviations arise from the non-sinusoidal nature of the oscillations. This introduces strong dependence on phase into oscillator phase velocity in the absence of perturbations. Such a dependence contradicts the definition of phase lying at the basis of Eq. (4.2), as in this equation phase increases uniformly in the absence of interactions. Moreover, this dependence swamps the effect of perturbations on the instantaneous rate of phase advance, see Fig. 4.4A. These phases obtained directly from the embeddings in Fig. 4.3 are thus referred to as $\theta$, the protophases. In order to isolate phase velocity changes resulting from perturbations, phase must be defined as increasing linearly 
in the absence of perturbations. A nearly linearly increasing phase is obtained with the help of the protophase probability density distribution $(2 \pi)^{-1} \sigma\left(\theta_{j}\right)$ [55], which is the inverse of the average instantaneous velocity of the oscillator through the limit cycle, see Fig. 4.4B. The genuine phases $\phi$ result from the transformations $d \phi_{j} / d \theta_{j}=\sigma\left(\theta_{j}\right)$, and exhibit non-linear phase advance in response to coupling or feedback only. This is evident in Fig. 4.4C, where the overall phase advance is uniform while localized excursions remain.

From the transformed, genuine phases $\phi_{j}$ we find the coupling functions $Q$, following the methods of [55]. First, the phase of each oscillator is cleansed of nonuniform phase advance. Then the phase velocities are fit with a two-dimensional Fourier expansion, Eq. (18) in [55] (when the phase space is not well-covered, phase velocities are instead fit with a two-dimensional kerneling function). The coupling functions are then further cleansed using the method in Section IV, part $B$ in [55]. Numerics demonstrate that satisfactory results can be obtained already after the first cleansing, since the second cleansing is small compared to the first. Note two limitations of the method (see [55] for details): (i) if the coupling function contains a component dependent only on the phase of the driven system, it will be cleansed; (ii) generally the coupling function contains a constant term which cannot be separated from the natural frequency; this may be done only if several observations with different yet unknown coupling strength are available. A matlab implementation of the techniques employed for the data analysis may be found online [67].

There is more than one way obtain the one-dimensional coupling functions, $q^{(j)}$. These can be obtained as in Eq. (4.6), by averaging from the two-dimensional coupling functions $Q^{(j)}$, or they can be obtained directly from the time series [3]. The methods are conceptually equivalent, and yield nearly identical results. The 
latter is easier to implement numerically, and we use it here to obtain $q^{(j)}$. First, the periods of each oscillation are calculated. The inverse of the period is the average frequency over the oscillation. Next, we calculate the average phase difference over each oscillation. Here we express phase difference as obtained from the genuine phases; phase differences from the protophases distort the coupling function if the oscillators are dissimilar. Finally, $q^{(j)}$ is obtained by fitting the average frequency as a function of phase difference. The fitting can be performed with a Fourier series or a kerneling function.

\subsection{Results}

Experiments were performed using the two-oscillator electrochemical system described in Section 4.4. Oscillator 1 has a relaxational waveform $\left(V_{1}=1.180 \mathrm{~V}\right)$ and oscillator 2 has a smooth waveform $\left(V_{2}=1.105 \mathrm{~V}\right)$. The oscillators are coupled using the form in Eqs. (4.9) and (4.10). Phase models of the two oscillators are then reconstructed from the genuine phase time series according to Eq. (4.2) or the procedure for the one-dimensional reconstruction discussed in Section 4.5.
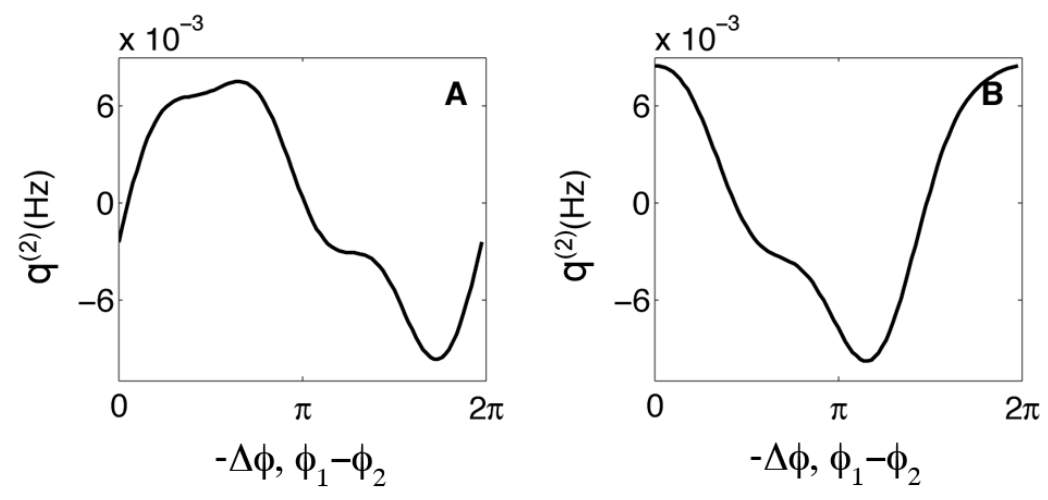

Figure 4.5: Coupling function for oscillator 2 based on phase difference, $q^{(2)}\left(\phi_{1}-\phi_{2}\right)$. (a) No time delay, $\tau=0$. (b) Time delay equal to roughly three quarters of oscillator 2 natural period, $\tau=1.8 \mathrm{~s}$. 

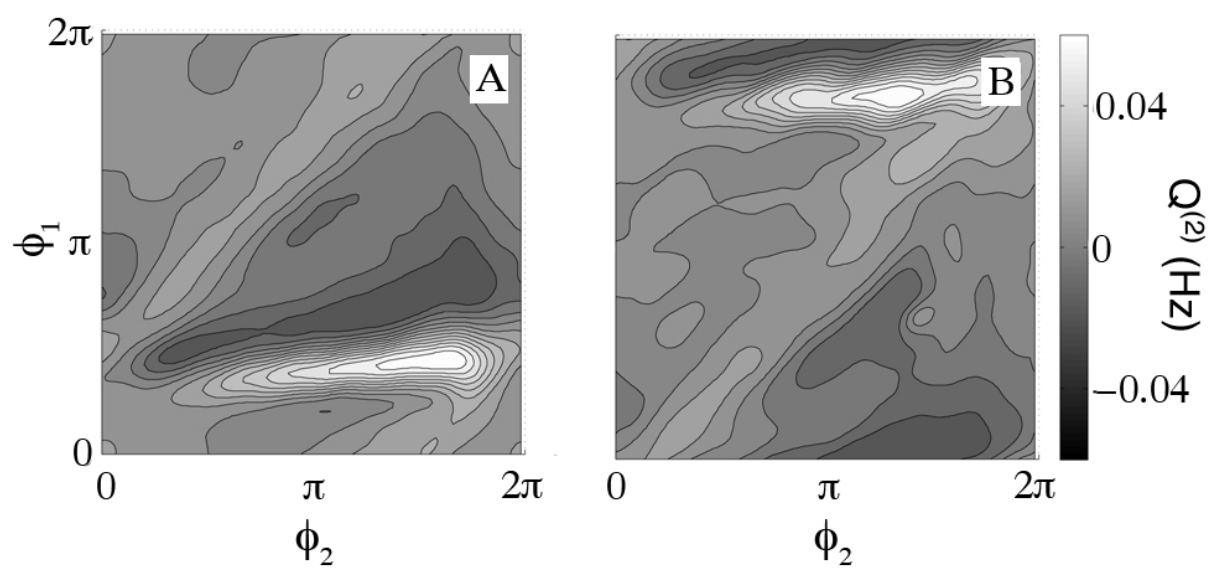

Figure 4.6: Coupling function in $\mathrm{Hz}$ for oscillator 2 based on each phase independently, $Q^{(2)}\left(\phi_{2}, \phi_{1}\right)$. (a) No time delay, $\tau=0$. (b) Time delay equal to three quarters the natural period of oscillator $2, \tau=1.8 \mathrm{~s}$.

In order to highlight the advantages of the two-dimensional coupling function, we compare the coupling functions of the one- and two-dimensional models with symmetrical coupling with and without time delay. Fig. 4.5A shows the onedimensional coupling function of oscillator 2 based on phase difference. The phase model of oscillator 1 is obtained in a similar fashion, but is not shown. This coupling function quantifies the oscillator's average change in frequency over a period. For example, when $\Delta \phi=\pi / 2$ the frequency of oscillator 2 increases relative to its natural frequency. Fig. 4.6A shows the two-dimensional coupling function of oscillator 2 based on each phase independently. The one-dimensional phase model, Fig. 4.5A, is the average of this two-dimensional model over trajectories between two crossings of $\phi_{2}=0$, i.e. one period. Note that the amplitude of the one-dimensional coupling function is an order of magnitude smaller than the two-dimensional due to the averaging. The two-dimensional function provides a mapping between instantaneous changes in phase velocity and the state of each system. For example, oscillator 2 advances most rapidly near $\phi_{1}=\pi / 2$ and $\phi_{2}=3 \pi / 2$.

Further experiments were performed in order to quantify the effects of time 
delay. From the definitions of the coupling function in Eqs. (4.4) and (4.6), time delay is expected to shift the stimulation function in the phase of the perturbing oscillator. Fig. 4.5B shows the coupling function of oscillator 2 based on phase difference for symmetric coupling and time delay of $\tau=1.8 \mathrm{~s}$. The phase model based on individual phases for this case is shown in Fig. 4.6B. Notice that both coupling functions are translated as expected. However, the two-dimensional coupling function clearly distinguishes between shifts in the two phases: Fig 4.6B becomes nearly identical to Fig 4.6A if one shifts the phase of the forcing oscillators $\phi_{1}$ by $\tau \nu_{1} 2 \pi=4.58 \mathrm{rad}$. Therefore, changes in coupling time delay are measurable from the two-dimensional model to within an additive factor of $2 \pi$. Time delay may be recovered from the one-dimensional model provided that the response function is known to be time-invariant.

Now we investigate the effect of changing coupling magnitude on the phase models. Focus is placed on the two-dimensional model, as it provides a more
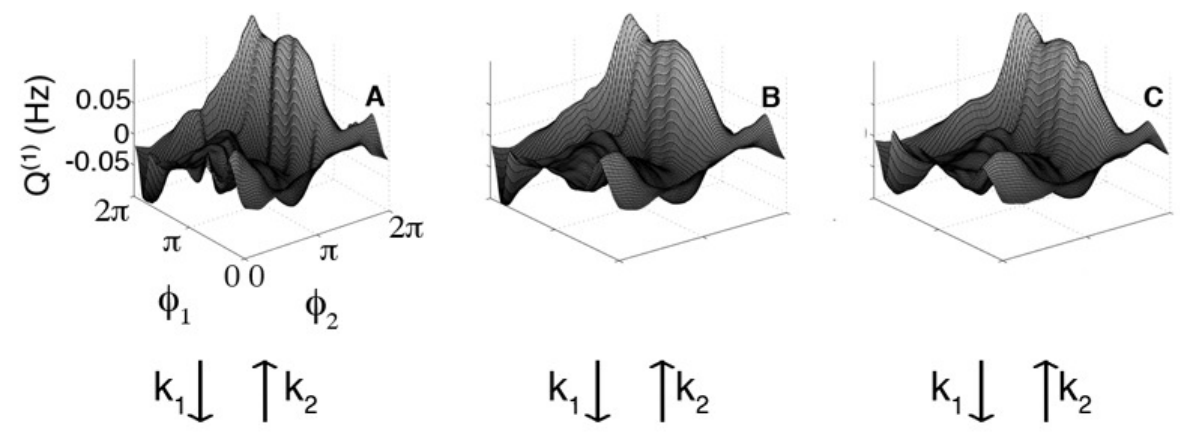

$k_{1} \downarrow \uparrow k_{2}$
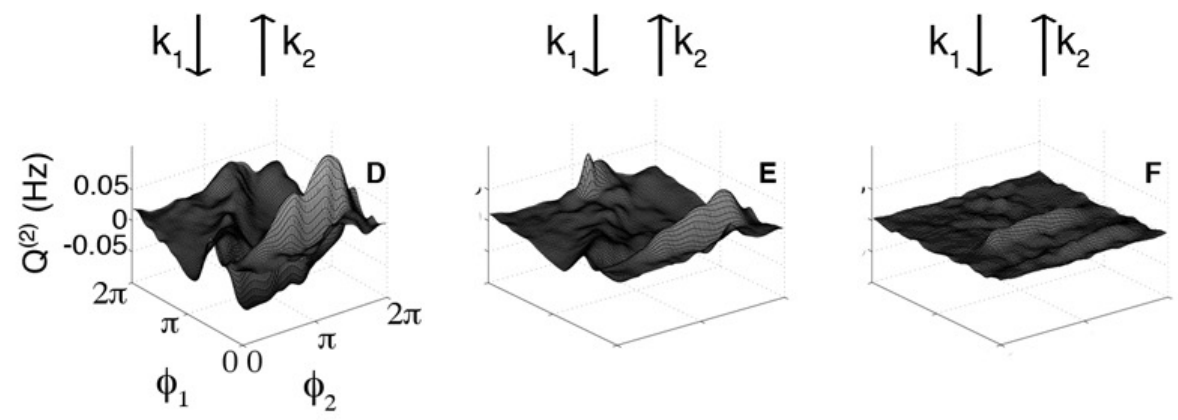

Figure 4.7: (top row) $Q^{(1)}\left(\phi_{1}, \phi_{2}\right)$ (bottom row) $Q^{(2)}\left(\phi_{2}, \phi_{1}\right) . k_{2}=1.0$ for all plots, $(\mathrm{a}, \mathrm{d}) k_{1}=1.0$, (b,e) $k_{1}=0.5$, (c,f) $k_{1}=0.1$. 
complete description of the system. Oscillator 1 is a relaxational oscillator $\left(V_{1}=\right.$ $1.210 \mathrm{~V}$ ) with a natural frequency of $0.42 \mathrm{~Hz} \pm 0.02 \mathrm{~Hz}$ and oscillator 2 is a smooth oscillator $\left(V_{2}=1.105 \mathrm{~V}\right)$ with a natural frequency of $0.53 \mathrm{~Hz} \pm 0.01 \mathrm{~Hz}$. Fig. 4.7 shows the coupling functions of oscillators 1 and 2 as a function of the genuine phases. The means of the coupling functions are less than one percent of the natural frequency, indicating a negligible change in average frequency due to coupling. Three coupling combinations $\left(k_{1}: k_{2}\right)$ are shown: symmetric, asymmetric (1:2) and highly asymmetric (1:10). As expected from Eq. (4.4), the amplitude of the surface variations decreases with diminishing stimulation magnitude. Also note that the functional dependence of each coupling function on its own phase is characteristic of the oscillator's response function [62].

The magnitude of an oscillator's response is quantified by the $L_{2}$-norm of its coupling function, $Q$. Fig. 4.8 shows the dependence of the coupling function norms on the coupling strengths $k_{1}$ and $k_{2}$. In this series of experiments the coupling strength to oscillator 2 was held constant at $k_{2}=1.0$ while the strength of the coupling to oscillator $1, k_{1}$, was increased incrementally from zero to 1.0. In a subsequent series of experiments with the same oscillators, the coupling strength on oscillator 1 was held constant at $k_{1}=1.0$ and $k_{2}$ was decremented from 1.0 to zero. Fig. 4.8 shows that the norm of the coupling function increases linearly with increasing coupling strength. This experimentally confirms that the coupling of electrochemical oscillators in the range of parameters studied is predominantly described by first-order terms in the coupling strength, i.e. we are in the regime of linear response.

The relative magnitudes of the coupling functions indicate the coupling directionality between the oscillators. In the electrochemical system described above, the values of $k_{1}$ and $k_{2}$, and therefore the relative coupling magnitude, are known 



Figure 4.8: (a) Relaxational oscillator: Norm versus gain $k_{1}$, linear fit: $\left\|Q^{(1)}\right\|=0.045 k_{1}+0.003, R^{2}=0.998$, (b) smooth oscillator: Norm versus gain $k_{2}$, linear fit: $\left\|Q^{(2)}\right\|=0.032 k_{2}+0.001, R^{2}=1.000$, where $R^{2}$ is the square of the correlation coefficient.

from the experimental setup. The applied directionality is,

$$
d_{A}=\frac{k_{1}-k_{2}}{k_{1}+k_{2}}
$$

Thus, $d_{A}$ varies between -1 and 1 . The more positive the directionality, the stronger $k_{1}$ is relative to $k_{2}$. A directionality of zero indicates equal coupling strengths, i.e. $k_{1}=k_{2}$. We can compare this quantity with the observed directionality, defined as

$$
d_{O}=\frac{C_{1}-C_{2}}{C_{1}+C_{2}},
$$

where $C_{j}=\left\|Q^{(j)}\right\| / \omega_{j}$. As shown in Fig. 4.8, the relaxational oscillator has a greater response to the same coupling gain and thus less voltage perturbation, according to Eq. (4.9). Therefore $d_{A}$ and $d_{O}$ are generally different. When the norms are scaled by the response slope from Fig. 4.8 , one restores $d_{O}=d_{A}$. Here we know $k_{1,2}$ and thus can obtain the response slopes; in general, $k_{1,2}$ may not be known.

Next we carried out experiments on an electrochemical oscillator with adjustable harmonic forcing. Fig. 4.9 shows the coupling functions, $Q^{(1)}$ and $q^{(1)}$, when the forcing frequency is $5 \%$ faster ( $\mathrm{A}$ and $\mathrm{C}$ ), and when the forcing frequency is $5 \%$ slower (B and D) than the natural frequency of the oscillator for a smooth 

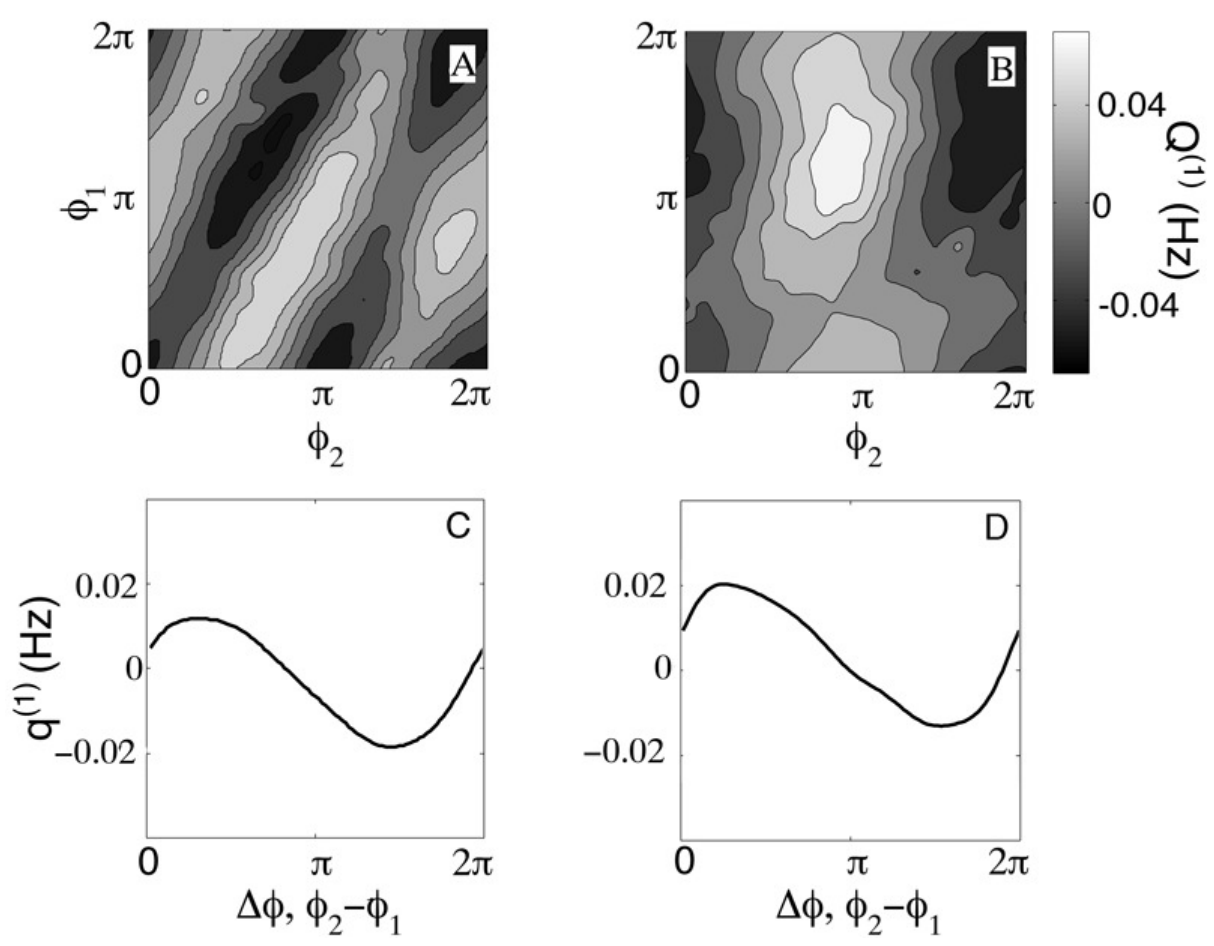

Figure 4.9: Experimental results, (a) $Q^{(1)}$ with $v / \omega=0.95$, (b) $Q^{(1)}$ with $v / \omega$ $=1.05$, (c) $q_{1,1}^{(1)}$ with $v / \omega=0.95$, (d) $q_{1,1}^{(1)}$ with $v / \omega=1.05$.

$(V=1.105 \mathrm{~V})$ oscillator. We see that the two-dimensional coupling function varies with forcing frequency, while the change is not detectable in the one-dimensional coupling function. The observed dependence of the two-dimensional coupling functions on the frequency of the driving has also been observed numerically with the harmonically forced van der Pol and periodic Rössler oscillators.

\subsection{Discussion}

In this paper we evaluate the coupling functions for two coupled nonlinear electrochemical oscillators directly from measured signals. We evaluate both the one$[3,20]$ and two-dimensional [55] coupling functions and compare the results of the models. While both models may recover changes in coupling time delay, only the 
two-dimensional model clearly distinguishes between changes in oscillator character and changes in time delay. We show that the relative magnitudes of the coupling functions quantify the directionality of coupling $[16,68]$. Using a toy model we show that the two-dimensional model predicts more synchronization regions and predicts the synchronization gain more accurately than the one-dimensional model. With an experimental oscillator driven by a harmonic voltage perturbation, we show that the two-dimensional model captures changes in the coupling function that are not detected by the one-dimensional coupling function.

We changed the coupling time-delay and calculated the one- and two-dimensional coupling functions (Figs. 4.5 and 4.6). The two-dimensional function indicates the precise configurations of both phases that correspond to maximum and minimum phase advance. Phase models based on generalized phase difference average out dependence of phase advance on the individual phases. Therefore in a onedimensional coupling function, a shift may be due to changes in time delay or in oscillator characteristics. Systems where the coupling and intrinsic system properties evolve with time include physiological and medical applications. Unless the oscillator is known to be time-invariant, the two-dimensional coupling function is the preferable model for inferring time delay.

We performed experiments in which we varied the ratio of the coupling components, $k_{1}$ and $k_{2}$ in Eqs. (4.9) and (4.10). We then calculated the two-dimensional coupling functions. Deviations from linear phase advance indicate points in phase space where an oscillator is susceptible to perturbation and is stimulated. This is nicely visualized in the top row of Fig. 4.7 which shows the instantaneous frequencies of the relaxational oscillator. There is a dominant ridge in the middle of the surface corresponding to maximum amplitude of the smooth oscillator, and therefore the greatest stimulation. A similar result is seen for the smooth oscillator 
in Fig. 4.7C. The largest instantaneous frequency on the ridge occurs near $\phi_{1}=3 \pi / 2$, which corresponds to the large amplitude in the oscillator phase-dependent response curve [62]. From the coupling functions, the coupling directionality was calculated, as in Eq. (4.13). The nearly linear increase in the norms of the coupling functions with gain, shown in Fig. 4.8, is a verification of the two-dimensional reconstruction.

Using the toy model of Eq. (4.7), we highlight the differences of the predictions of the one- and two-dimensional models. We construct two Arnold tongues (Fig. 4.1A and $\mathrm{B}$ ), and show that the synchronization gains predicted by the two models differ increasingly as the forcing frequency becomes farther from the resonance condition. Additionally, we show that the two-dimensional model predicts many regions of synchrony in a Devil's staircase (Fig. 4.1C); the one-dimensional model predicts only two regions of synchrony. The differences between the predictions of the two models in a relatively simple and explicitly defined system illustrate how the two models may differ in more complex systems.

Finally, we show from experimental data that the two-dimensional coupling function depends upon the forcing frequency, while the one-dimensional coupling function does not (Fig. 4.9). As already mentioned, the phase approximation is valid if the cycle is sufficiently stable and therefore the amplitudes can be considered as fixed. For this case the coupling function $Q^{(i)}$ can be reconstructed from data. If the coupling is sufficiently small, this function can be approximated by only one term of the series Eq. (4.3), i.e. $Q^{(j)}\left(\phi_{j}, \phi_{n}\right) \approx \varepsilon Q_{1}^{(j)}\left(\phi_{j}, \phi_{n}\right)$, and in this approximation the function depends solely on the phases, but not on the frequencies. However, the condition when the first approximation suffices is not yet known, and if it is not fulfilled, we can expect a dependence on the frequency and on the amplitude of the forcing. In this experiment, neither the stimulation function nor the response 
function changes, so we may infer according to Eq. (4.4) that the first-order approximation does not hold. The effect of forcing frequency on the coupling function was not previously predicted or shown; this effect as well as the range of applicability of the first-order approximation represent opportunities for further study.

An interesting and practically important problem is determination of the response function $Z_{j}\left(\phi_{j}\right)$ from the observation of the driven system. The ability to separate stimulation and response could be useful in any system where coupling evolves over time, such as system where learning occurs [13, 23]. When the first order approximation is valid, as in Eq. (4.4), the coupling function can be represented as a product $Q_{1}^{(j)}\left(\phi_{j}, \phi_{j}\right)=Z_{j}\left(\phi_{j}\right) h_{j}\left(\phi_{n}\right)$. Because there is indication of significant higher order terms in the coupling function, the first-order approximation is not valid here and the deconvolution is not possible. We can suggest an alternative explanations for this; the driving may enter the state-space Eqs. (4.1) as a multiplicative term, e.g. as $f\left(x_{1}\right) g(v t)$; in the process of phase reduction this term yields a function of two phases which cannot be written as a product of two one-dimensional functions. This important issue also requires further studies.

\subsection{Acknowledgements}

This work was supported in part by the National Science Foundation (CBET0730597). 


\section{Chapter 5}

\section{Clustering in small networks with}

\section{delay $^{1}$}

\subsection{Notes to the reader}

This chapter closely mirrors our recently accepted paper with Eckehard Schöll's group [69]. Here, we select sets of four oscillators from the whole population of 64; we apply time-delayed coupling to induce phase locking. To obtain interaction functions, shown in Figure 5.5, we weakly coupled pairs of oscillators. Please note that Section 5.4 describing the experimental apparatus is repeated from Chapter 3. We repeat this information for the convenience of the reader. Also note that Section 5.8, the discussion of this chapter, has been re-written for greater readability.

\footnotetext{
${ }^{1}$ K. A. Blaha, J. Lehnert, A. Keane, T. Dahms, P. Hövel, E. Scholl and J. L. Hudson, “Clustering in delay-coupled smooth and relaxational chemical oscillators" Phys. Rev. E, (accepted).
} 


\subsection{Introduction}

The field of nonlinear dynamics in coupled systems has seen a huge increase in interest during the last years $[41,57,70-72]$. The systems range from a few coupled elements to complex networks. Collective dynamics may arise in various patterns, of which in-phase (or zero-lag) synchronization is the most prominent. Traveling waves $[73,74]$ and cluster or group synchronization [75-77] are other examples. In particular, during cluster synchronization, parts of a network synchronize with zero lag, but with a nonzero phase-lag between different clusters. Interest in cluster synchrony has led to significant theoretical [75-80] and experimental [81-85] results.

A recent theoretical study [49] discussed cluster synchronization in delaycoupled networks of Hopf normal-form oscillators (also known as Stuart-Landau oscillators), which are given by a generic model of a Hopf bifurcation. Depending upon the delay time, different cluster states exist and are stable. These intervals overlap leading to multistable regimes; i.e., the specific state that is realized is determined by the initial conditions. In Ref. [49] it was shown that the phase of a complex coupling constant can be used to select a desired cluster state.

Combining theoretical analysis with experiments, chemical oscillators can be mathematically described by very simple models like the Kuramoto phase oscillator model or the Stuart-Landau model in certain regimes of operation. In the present paper, we study a system of four chemical oscillators coupled in a unidirectional ring.

We consider two regimes of operation: (1) For low bias voltages, the elements show smooth sinusoidal oscillations and we are able to verify the results of Ref. [49] experimentally. The theory not only correctly predicts the interval where each 
cluster state exists but also quantitatively the common frequency which arises depending upon the delay time and the particular cluster state. We show that the time delay can be used to ensure the existence and stability of a desired cluster state, instead of the phase of a complex coupling constant, which is unavailable in the experiment. (2) For a higher bias voltage, the oscillations become of the strongly nonlinear relaxational type. In this regime, cluster synchronization with secondary cluster states, i.e., unequal phase differences between the clusters, can occur in addition to symmetric cluster states. We introduce an extended version of the StuartLandau model, based on experimentally measured interaction functions. Linear stability analysis and numerical continuation allow for a theoretical treatment of these states.

This paper is organized as follows: Sec. 5.3 presents a short summary of relevant results from Ref. [49]. We introduce the theoretical model and carry out the analysis that allows us to predict intervals of existence and common frequencies of the different cluster states. Sec. 5.4 describes the experiment using chemical oscillators. Sec. 5.5 presents the corresponding experimental results for smooth oscillators. Having identified limitations in the existing theory, we adapt the theoretical model for more general use and apply it again to the chemical oscillators in the relaxational regime using a higher bias voltage in Sec. 5.6 and Sec. 5.7. Details of the extended theory are given in Sec. 5.7.1. Finally, we conclude with Sec. 5.8.

\subsection{Theoretical model: Sinusoidal Oscillations}

This section summarizes the results of Ref. [49], concerning cluster synchronization and stability in a network of $N$ Stuart-Landau oscillators. We focus on the case that is relevant to the considered experimental setup (see Sec. 5.4), namely a topology 
given by a unidirectionally coupled ring and the case of real coupling constant. In Ref. $[49,86]$ more general topologies and complex coupling constant were discussed as well.

The dynamics of the Stuart-Landau oscillators in a unidirectionally coupled ring is given by:

$$
\dot{z}_{j}=f\left(z_{j}\right)+K z_{(j+1) \bmod N}(t-\tau)
$$

with $z_{j}=r_{j} e^{i \varphi_{j}} \in \mathbb{C}, j=1, \ldots, N$, time delay $\tau$, and coupling strength $K$. For notational convenience, we will drop the modulus $N$ in the indices in the following, i.e., $z_{j+1} \equiv z_{(j+1) \bmod N}$. Note that in Ref. [49] a diffusion-like coupling was used. There, the coupling term reads $z_{j+1}(t-\tau)-z_{j}$ instead of just $z_{j+1}(t-\tau)$. Nevertheless, this alters the involved equations only slightly so that we only have to adapt the analysis of Ref. [49] at a few points.

The local dynamics of each element is given by the normal form of a supercritical Hopf bifurcation:

$$
f(z)=\left[\lambda+i \omega-(1+i \gamma)|z|^{2}\right] z
$$

with real constants $\lambda, \omega \neq 0$, and $\gamma$. This class of systems arises naturally as a generic expansion in center manifold coordinates near a Hopf bifurcation, and therefore its dynamics is generic for many systems close to the Hopf bifurcation.

In polar coordinates with radius and phase variables the system Eq. (6.6) reads as follows:

$$
\begin{gathered}
\dot{r}_{j}=\left(\lambda-r_{j}^{2}\right) r_{j}+K r_{j+1}(t-\tau) \cos \left(\varphi_{j+1}(t-\tau)-\varphi_{j}(t)\right) \\
\dot{\varphi}_{j}=\omega-\gamma r_{j}^{2}+K \frac{r_{j+1}(t-\tau)}{r_{j}} \sin \left(\varphi_{j+1}(t-\tau)-\varphi_{j}(t)\right) .
\end{gathered}
$$

Cluster states with a common amplitude and equal phase lags between neigh- 
boring nodes can be described by $r_{j} \equiv r_{0, m}$ and $\varphi_{j}=\Omega_{m} t+j \Delta \varphi_{m}$ with collective frequency $\Omega_{m}$ and $\Delta \varphi_{m}=2 \pi m / N$. Such states we call primary states. In contrast, the nodes of secondary states as discussed in Section 5.7 are still characterized by a common collective frequency, but different amplitudes and phase lags. The integer $m=0, \ldots, N-1$ labels the specific states: In the case of four nodes, there are four possible cluster states: $m=0$ corresponds to zero-lag synchronization, $m=1$ is the splay state, $m=2$ the 2 -cluster state, while $m=3$ labels the reverse splay state (see below). Applying this notion to Eqs. (6.8) yields the following set of transcendental equations for the collective amplitude $r_{0, m}$ and frequency $\Omega_{m}$ of the $m$-state:

$$
\begin{aligned}
& r_{0, m}^{2}=\left(\lambda+K \cos \Phi_{m}\right) \\
& \Omega_{m}=\omega-\gamma r_{0, m}^{2}+K \sin \Phi_{m},
\end{aligned}
$$

where $\Phi_{m}=\Delta \varphi_{m}-\Omega_{m} \tau$. Note that $\Omega_{m}$ only depends on $r_{0, m}$ if $\gamma \neq 0$. Thus, the parameter $\gamma$ couples the frequency to the oscillation amplitude (anisochronicity).

Considering small deviations $\delta r_{j}$ and $\delta \varphi_{j}$, we obtain $r_{j}=r_{0, m}\left(1+\delta r_{j}\right), \varphi_{j}=$ $\Omega_{m} t+j \Delta \varphi_{m}+\delta \varphi_{j}, \xi_{j}=\left(\delta r_{j}, \delta \varphi_{j}\right)^{T}$. This leads to a variational equation for the $m$-cluster state:

$$
\dot{\xi}=\mathbf{I}_{N} \otimes\left(\mathbf{J}_{0, m}-K \mathbf{R}_{m}\right) \xi+K\left(\mathbf{G} \otimes \mathbf{R}_{m}\right) \xi(t-\tau)
$$

with the $2 N$-dimensional vector $\xi=\left(\xi_{1}, \ldots, \xi_{N}\right)^{T}$, the $N \times N$ identity matrix $\mathbf{I}_{N}$, and matrices $\mathbf{R}_{m}=\left(\begin{array}{cc}\cos \Phi_{m} & -\sin \Phi_{m} \\ \sin \Phi_{m} & \cos \Phi_{m}\end{array}\right), \mathbf{J}_{0, m}=\left(\begin{array}{cc}-2 r_{0, m}^{2} & 0 \\ -2 \gamma r_{0, m}^{2} & 0\end{array}\right)$. The adjacency matrix $\mathbf{G}$ describes the unidirectional ring topology: $g_{j n}=1$ for $n=j+1$ and zero otherwise. Diagonalizing G, we arrive at the block-diagonalized variational equation:

$$
\dot{\zeta}_{k}(t)=\mathbf{J}_{0, m} \zeta_{k}(t)-K \mathbf{R}_{m}\left[\zeta_{k}(t)-v_{k} \zeta_{k}(t-\tau)\right]
$$


where $v_{k}=e^{2 i k \pi / N}, k=0,1, \ldots, N-1$, are the eigenvalues of G. Note that Eq. (5.6) can be considered as a master stability equation [47] for Eq. (6.6). Here, the coefficient matrices $\mathbf{J}_{0, m}$ and $\mathbf{R}_{m}$ do not depend on time. Hence, the Floquet exponents of the synchronized periodic state are given by the eigenvalues $\Lambda$ of the characteristic equation

$$
\operatorname{det}\left\{\mathbf{J}_{0, m}-\Lambda \mathbf{I}_{2}+K\left(-1+e^{2 i k \pi / N-\Lambda \tau}\right) \mathbf{R}_{m}\right\}=0 .
$$

If for all $k=0, \ldots, N-1$ all Floquet exponents (except the one relating to the Goldstone mode) have a negative real part, the cluster state with index $m$ will be stable.

Figure 5.1 depicts the common frequencies of all four possibles cluster states in

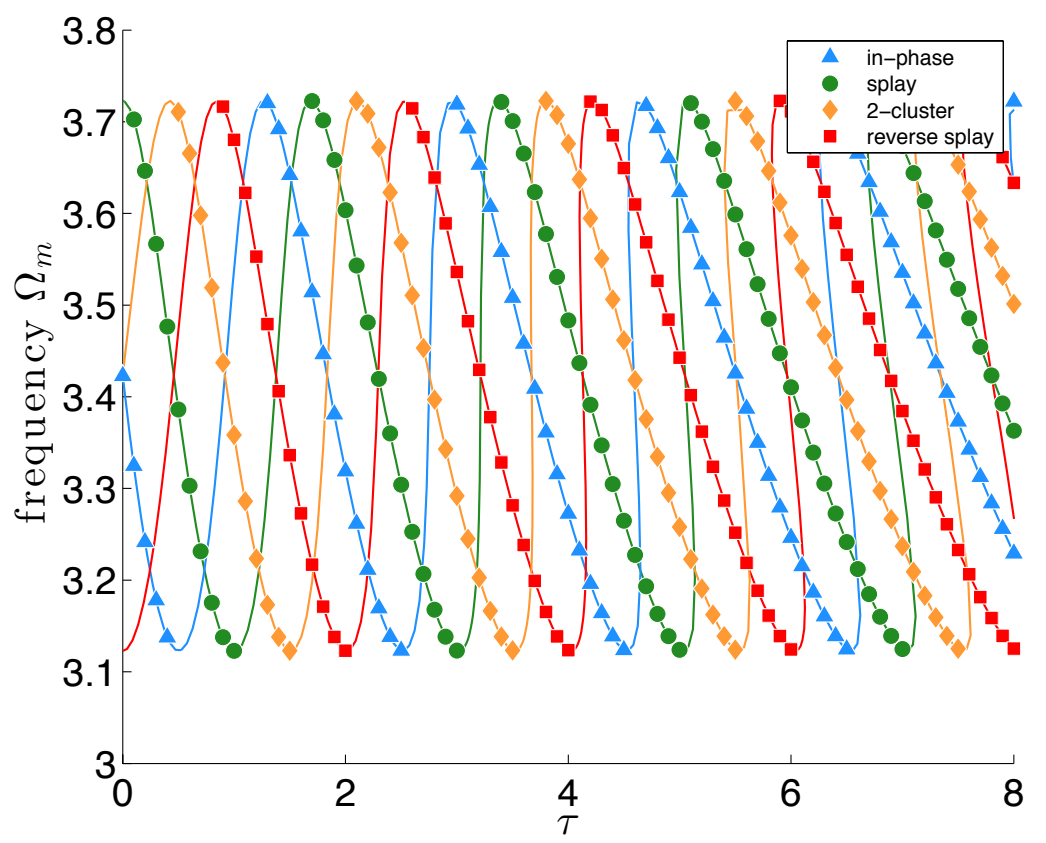

Figure 5.1: The frequencies $\Omega_{m}$ of the four possible cluster states as time delay, $\tau$ varies, as given by solutions of Eqs. (5.4). Solutions may be stable or unstable. Stable solutions: blue triangle: in-phase state. green circle: splay state. orange diamond: 2-cluster state. red square: reverse splay state. Blank curves refer to unstable solutions. The stability is determined via Eq. (5.7). Parameters: $\lambda=1.1025, \omega=3.4228, \gamma=0, K=0.3, N=4$. 
a unidirectionally coupled ring of four elements. Symbols and blank curves refer to stable and unstable solutions, respectively, where the stability is calculated via the characteristic equation (5.7). It can be seen that for most values of $\tau$ multistability between different states exists, but that all four cluster states do not necessarily occur for every value of $\tau$. However, it is always possible to choose the delay in such a way that the desired $m$-state exists with a frequency $\Omega_{m}$. Using the delay time

$$
\tau_{\mathrm{m}}=\frac{2 \pi m}{N \Omega_{m}}
$$

Eq. (5.4b) holds for $\Phi_{m}=0$, so that $\Omega_{m}=\omega-\gamma(\lambda+K)$. From $\Phi_{m}=0$ we also have $\mathbf{R}_{m}=\mathbf{I}_{2}$, meaning that Eq. (5.7) simplifies to:

$$
\begin{aligned}
{\left[-2 r_{0, m}\right.} & \left.+K\left(-1+e^{2 i k \pi / N-\Lambda \tau}\right)-\Lambda\right] \\
& \times\left[K\left(-1+e^{2 i k \pi / N-\Lambda \tau}\right)-\Lambda\right]=0 .
\end{aligned}
$$

The dominant Floquet exponent is always obtained by setting the second factor to zero: $\Lambda=K\left(-1+e^{2 i k \pi / N-\Lambda \tau}\right)$. The solution $\Lambda$ of this equation will always have a negative real part [49]. Note that the choice $\tau_{\mathrm{m}}$ only guarantees the existence and stability of the $m$-state, but does not ensures monostability. In fact, for the parameter choice of Fig. 5.1 and generally for large enough delay times, multistability clearly persists for $\tau=\tau_{\mathrm{m}}$.

\subsection{Experimental setup}

The experimental setup is described in the following. Experiments are performed in an electrochemical cell consisting of four 1-mm diameter Ni working electrodes (99.98\% pure), a Pt mesh counter electrode, and $\mathrm{Hg} / \mathrm{Hg}_{2} \mathrm{SO}_{4} / \mathrm{K}_{2} \mathrm{SO}_{4}$ (sat) reference 
electrode, with a $3 \mathrm{M} \mathrm{H}_{2} \mathrm{SO}_{4}$ electrolyte, shown in Fig. 5.2(a). The four electrodes are electrically coupled in a unidirectional ring. The cell is enclosed in a jacketed glass vessel maintained at a temperature of $11^{\circ} \mathrm{C}$. An ACM Instruments multi-channel potentiostat is used to set the potentials $V_{0}$ of the electrodes such that they undergo transpassive dissolution. A resistor, $R_{p}=650 \Omega$, is attached to each electrode, causing the dissolution currents $I_{j}$ to oscillate [52]. These resulting electrodissolution currents are measured at $250 \mathrm{~Hz}$ using zero resistance amperemeters (ZRAs) attached to a real time data acquisition system.
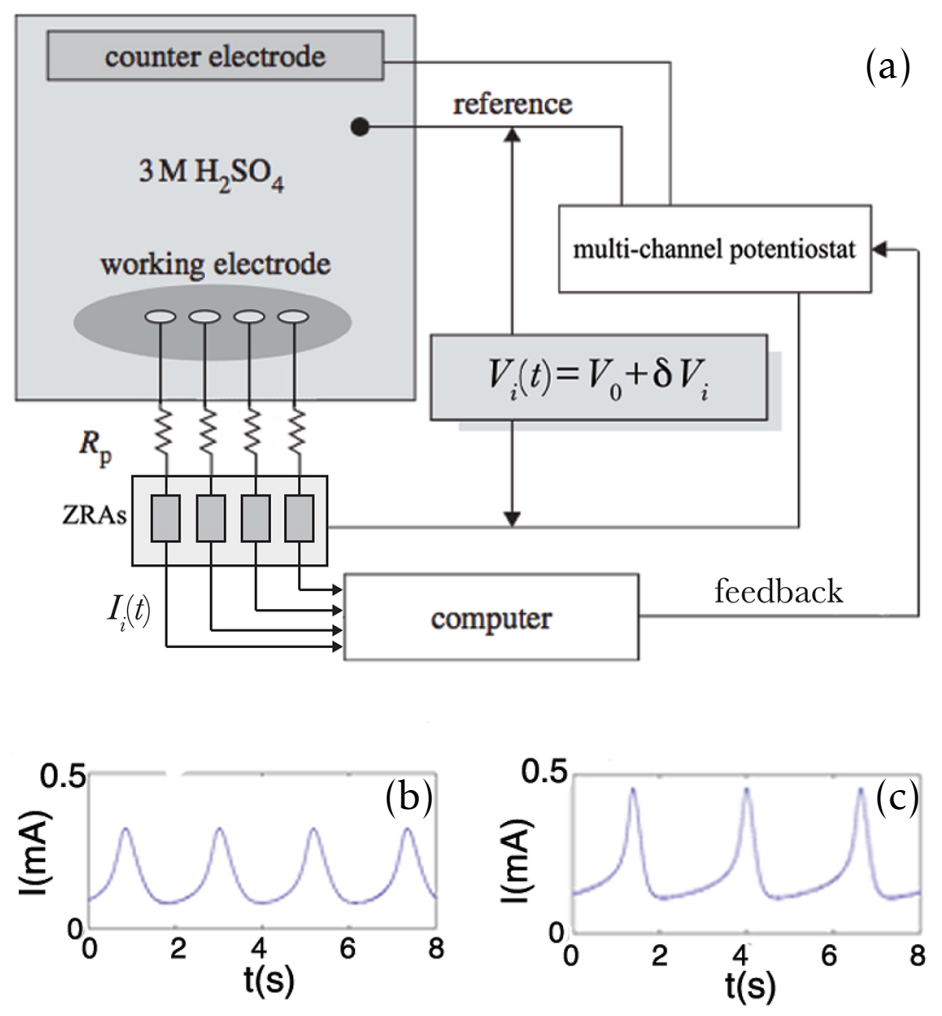

Figure 5.2: (a) Experimental apparatus with multi-channel addressable feedback, $R_{p}$ is the channel resistance of $650 \Omega$. (b) Electrochemical dissolution time series showing smooth oscillators at a potential of $V_{0}=1.105 \mathrm{~V}$. (c) Time series of relaxation oscillators at $V_{0}=1.2 \mathrm{~V}$. ZRA: zero resistance amperemeter.

Four oscillators with similar frequencies are selected from an array of 64 oscilla- 
tors. The character of the oscillator may be varied by the choice of applied voltage. Experiments are performed at two voltages: a low voltage to induce nearly-harmonic oscillations, which can be modeled by Stuart-Landau oscillators (cf. Sec. 5.5), and a higher voltage exhibiting higher harmonics and more complex behavior (cf. Sec. 5.7). We will use the terms smooth and relaxation oscillations, respectively. Negligible intrinsic electrical interactions exist between the uncoupled oscillators [19]. The startup or shutdown of an oscillator does not alter the behavior of the other oscillators. Furthermore, the oscillator dynamics has no interdependence when oscillators are functioning in the uncoupled state.

Interactions are introduced using real-time coupling of the form

$$
V_{j}(t)=V_{0}+\delta V_{j}(t)
$$

where $\delta V_{j}$ are the changes in the circuit potentials of the $j$ th elements due to the feedback. These feedback voltages are given by

$$
\delta V_{j}(t)=K \sum_{n=1}^{N} g_{j n}\left[V_{n}(t-\tau)-R_{p} \hat{I}_{n}(t-\tau)\right],
$$

where $R_{p}=650 \Omega$ is the channel resistance, $K$ is the fixed overall coupling gain, and $\tau$ denotes the coupling time delay, which is realized by the real-time data acquisition system combined with the multi-channel potentiostat. $\hat{I}_{n}$ are the normalized currents measured by the ZRAs.

To obtain this quantity, the measurements of the dissolution current $I_{n}$ are first scaled such that the mean value of each channel $\bar{I}_{n}$ is removed as an offset. Then, we perform normalization of the amplitude of the oscillation $I_{n}^{\max }$ relative to the mean amplitude of the electrode ensemble $N^{-1} \sum_{n=1}^{N} I_{n}^{\max } \cdot g_{j n}$ is an element of the adjacency matrix G, which describes the structure of the coupling. We apply unidirectional 
coupling on a four-member ring with the adjacency matrix

$$
\mathbf{G}=\left(\begin{array}{llll}
0 & 1 & 0 & 0 \\
0 & 0 & 1 & 0 \\
0 & 0 & 0 & 1 \\
1 & 0 & 0 & 0
\end{array}\right) .
$$

This coupling scheme is implemented via the multi-channel potentiostat (see Fig. 5.2).

We calculate the dynamical variables (amplitudes $r_{j}$ and phases $\varphi_{j}$ ) from the ZRAs' experimental measurement of the electrodissolution current of each oscillator (see Fig. 5.2). From these currents, the phase of each oscillator is found by peak-topeak linear interpolation, where the peak is defined as 0 or $2 \pi$ [87]. From the phases, we can then calculate the average frequencies of the oscillators. The amplitudes are measured as half of the difference between the peak and trough value of the electrodissolution current, giving one data point per period.

The parameters $\lambda$ and $\omega$ that belong to the theoretical model can be identified by the dynamics of a single uncoupled oscillator (see Figs. 5.2(b) and (c)). $\lambda$ must be set such that the amplitude $r$ is equal to $\sqrt{\lambda}$; while $\omega$ should be chosen such that the period of oscillation must be equal to $2 \pi / \omega$. In the experiments $\omega$ is not identical for each oscillator, but the oscillators are chosen so that the values of $\omega$ are very close to each other. As such $\omega$ is simply taken to be the average (i.e. $\omega=N^{-1} \sum_{j=1}^{N} \omega_{j}$ ). Furthermore, we let $\gamma$ be equal to zero, i.e., the frequency does not depend on the radius. 


\subsection{Smooth oscillations}

In this section we consider the case of smooth sinusoidal oscillations. The four electrodes were held at a voltage of $V_{0}=1.105 \mathrm{~V}$. This is slightly above a Hopf bifurcation that occurs at $V_{0} \approx 1.05 \mathrm{~V}$. Hence the oscillations are nearly harmonic (cf. Fig. 5.2(b)). Although visually the time series is not perfectly sinusoidal, in practice we can model the oscillators' phase dynamics as linear [62].

The four oscillators operate at $\omega_{1}=3.424 \pm 0.063 \mathrm{rad} / \mathrm{s}, \omega_{2}=3.393 \pm 0.069 \mathrm{rad} / \mathrm{s}$, $\omega_{3}=3.418 \pm 0.063 \mathrm{rad} / \mathrm{s}$, and $\omega_{4}=3.456 \pm 0.057 \mathrm{rad} / \mathrm{s}$. The frequency range is due to the slow drift of the natural frequencies of the oscillators.

Figure 5.3 depicts the measured and numerically calculated, stable states of the compound system depending upon the time delay in panels (a) and (b), respectively. In Fig. 5.3(b), lines are calculated from Eqs. (5.4) and points are based on solution continuation of Eqs. (6.8) using DDE-BIFTOOL. In order to resolve the multistability present in the coupled system we slowly increase the time delay (shown by arrows in Fig. 5.3(a)) up to $\tau=1.25 \times(2 \pi / \omega)$ at which point we perform a down-ramping. During the up-sweep, $\tau$ is increased from $0.80 \times(2 \pi / \omega)$ to $1.25 \times(2 \pi / \omega)$ in increments of $0.05 \times(2 \pi / \omega)$. The system is allowed to reach a stationary state at each value of $\tau$. The qualitatively different states are marked by the following symbols: red squares represent the reverse splay state, blue triangles represent the in-phase state, and green circles represent the splay state.

We start at $\tau=0.8 \times(2 \pi / \omega)$ with a reverse splay state, which is characterized by phase difference of $3 \pi / 2$ between two subsequent oscillators. Increasing to $\tau=0.95 \times(2 \pi / \omega)$, we obtain in-phase synchronization with $\varphi_{1}=\varphi_{2}=\varphi_{3}=\varphi_{4}$. For larger $\tau$ values, the system exhibits a splay state; that is, phase difference is $\pi / 2$ between two subsequent oscillators. 


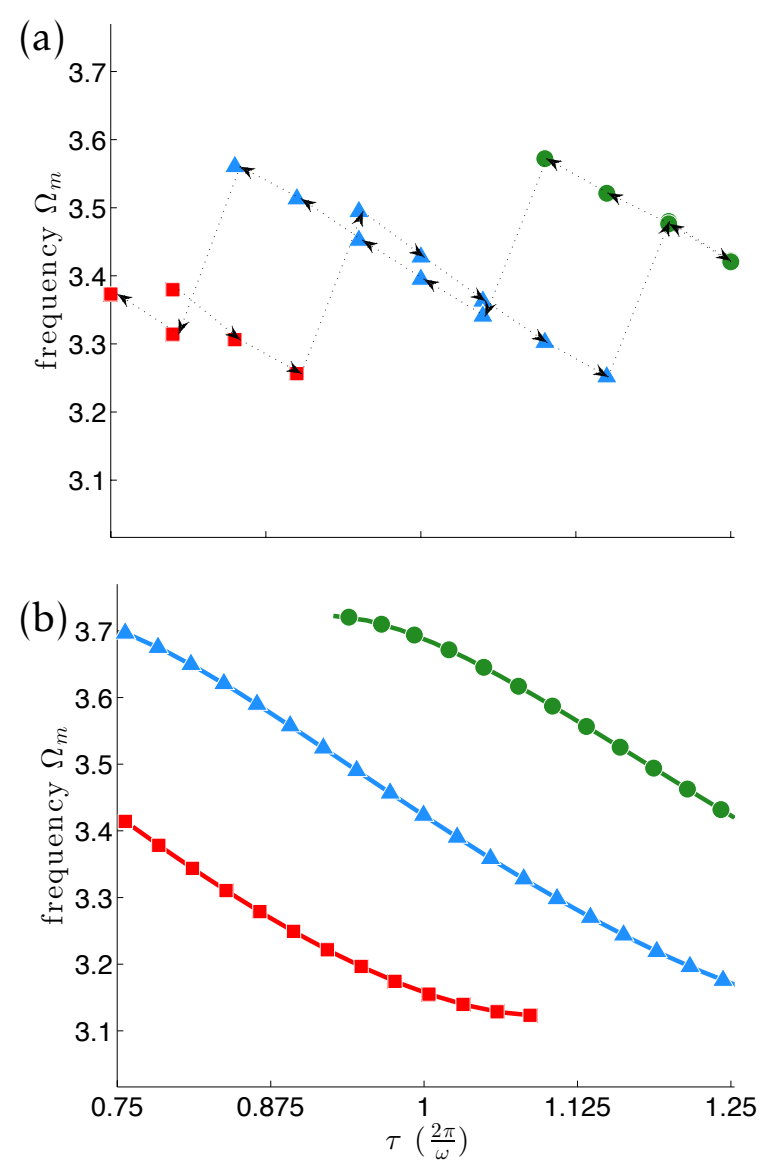

Figure 5.3: Collective frequency $\Omega_{m}$ versus the time delay $\tau$. Red squares, blue triangles, and green circles represent a reverse splay state, an in-phase state, and a splay state, respectively. (a) Experimental data, $V_{0}=1.105 \mathrm{~V}$, $K=0.15$. The arrows indicate an increase or decrease of $\tau$ during the measurement. (b) Solution continuation of Eqs. (6.8) using DDE-BIFTOOL (symbols) and numerical solutions of Eqs. (5.4) (lines). Parameters as in Fig. 5.1.

During the down-sweep, the time delay is decremented by $0.05 \times(2 \pi / \omega)$ until $\tau=0.75 \times(2 \pi / \omega)$. We observe the same states in the down-sweep as the up-sweep. Each time $\tau$ is decremented, the frequency increases slightly, except when a cluster transition occurs and the frequency abruptly jumps to a lower value. The system maintains the splay state until transitioning to the in-phase cluster state and then the reverse splay state. The transitions are also shown by arrows for the up- and 
down-sweep. Note that they occur at different time delays depending upon the direction of the time delay sweep. The coexistence of several cluster states at a given value of $\tau$, which depends upon the prior state of the system, demonstrates hysteresis. In Fig. 5.3(a) showing the experimental data, the triangles representing the in-phase cluster state are slightly nonidentical near $\tau=1 \times(2 \pi / \omega)$; this is due to drift in the natural frequencies during the course of the experiment.


Figure 5.4: Experimental time series (top) and schematic diagram (bottom) of an in-phase state (a), a 2-cluster state (b), a reverse splay state (c), and a splay state (d) as shown in Fig. 5.3(a). The schematics show phase relations between oscillators on the phase ring. Oscillator colors in the schematic correspond to the colors in the times series: $\varphi_{1}$ is shown in blue (solid), $\varphi_{2}$ is black (dashed), $\varphi_{3}$ is red (dotted), and $\varphi_{4}$ is green (dot-dashed). Parameters: $V_{0}=1.105 \mathrm{~V}, K=0.15$; time delays: (a) $\tau=1.05 \times 2 \pi / \omega$, (b) $0.5 \times 2 \pi / \omega$, (c) $1.2 \times 2 \pi / \omega$, and (d) $0.8 \times 2 \pi / \omega$ with $\omega=3.4228 \mathrm{rad} / \mathrm{s}$ as in Fig. 5.1, respectively.

Figures 5.4(a)-(d) illustrate the qualitative differences between the cluster states seen in Fig. 5.3. Next to the time series, corresponding schematic diagrams are also depicted. Note, that the 2-cluster state (Fig. 5.4(b)) does not occur in the range of $\tau$ 
shown in Fig. 5.3.

We see that the cluster states and hysteresis can be modeled by the Stuart-Landau oscillator as given in Eqs. (6.8). The numerical results, including simulations as well as path continuation using DDE-BIFTOOL $[88,89]$, shown in Fig. 5.3(b) closely match the experimental results. The only discrepancy seen is that the branches of each cluster state in the experiments seem to be stable for a shorter range of $\tau$, leading to an earlier jump to another cluster state. This is probably due to experimental noise and small heterogeneities in the oscillators' parameters.

\subsection{Interaction functions}

If we increase the voltage to $V_{0}=1.2 \mathrm{~V}$, the profile of the oscillations deforms from a sinusoidal to a strongly nonlinear relaxation oscillation. One could use a nonlinear time transformation to map the relaxation oscillations back to the sinusoidal model and thus still employ the Stuart-Landau model for a theoretical description. However, this nonlinear time transformation will also affect the coupling, which no longer can be assumed to be sinusoidal. Instead we rewrite Eqs. (6.8) in a more general form

$$
\begin{aligned}
\dot{r}_{j} & =\left(\lambda-r_{j}^{2}\right) r_{j}+K r_{j+1}(t-\tau) H_{r}\left(\varphi_{j+1}(t-\tau)-\varphi_{j}(t)\right) \\
\dot{\varphi}_{j} & =\omega+K \frac{r_{j+1}(t-\tau)}{r_{j}} H_{\varphi}\left(\varphi_{j+1}(t-\tau)-\varphi_{j}(t)\right),
\end{aligned}
$$

where $H_{r}$ and $H_{\varphi}$ are coupling functions, also called interaction functions, which can be obtained experimentally.

To determine $H_{r}$ and $H_{\varphi}$, we conduct a separate, but related experiment according to the methods described in Refs. [20,33, 87]. We select two oscillators 

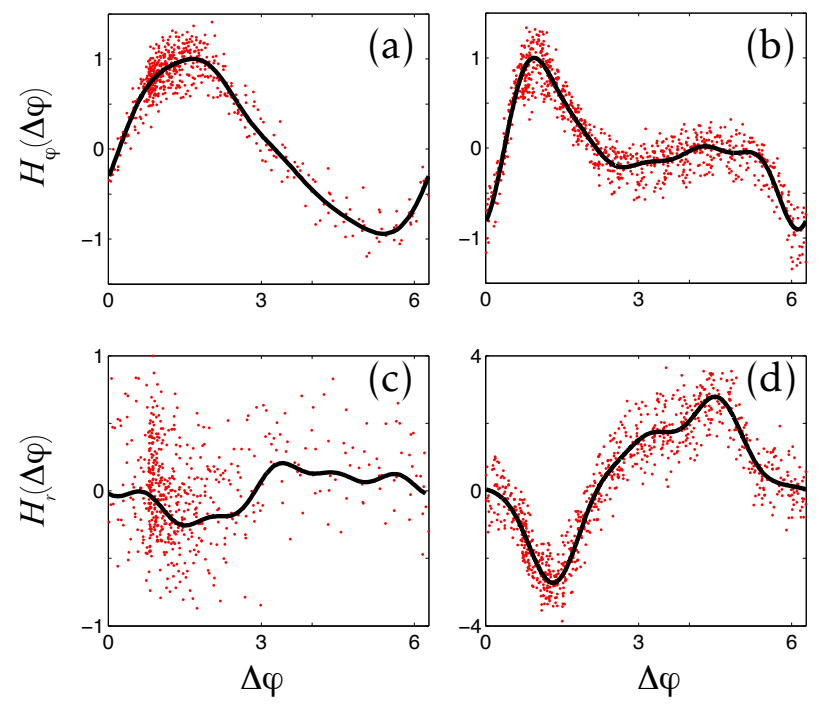

Figure 5.5: Phase interaction function $H_{\varphi}$ in panels (a) and (b) for $V_{0}=$ $1.105 \mathrm{~V}$ and $V_{0}=1.2 \mathrm{~V}$, respectively; radial interaction function $H_{r}$ in panels (c) and (d) for $V_{0}=1.105 \mathrm{~V}$ and $V_{0}=1.2 \mathrm{~V}$, respectively. Experimentally obtained data is shown in red dots. A 5-term Fourier fit from evenly sampled data is shown by the black curve.

at the same voltage with slightly different natural frequencies and couple them such that they interact, but do not phase lock. We measure the time-dependent radius $r_{j}\left(\varphi_{i}(t)-\varphi_{j}(t)\right)$ and the frequency $\Omega_{j}\left(\varphi_{i}(t)-\varphi_{j}(t)\right)$ of oscillator $j$ as a function of phase difference $\Delta \varphi=\varphi_{i}(t)-\varphi_{j}(t)$. We find that $r_{j}$ and $\Delta \varphi$ are approximately constant over one oscillation period. Following Ref. [33] with stationary radii $r_{j}$, we use

$$
H_{\varphi}(\Delta \varphi)=-\frac{2 \pi \Delta T_{j}}{T_{j}^{2}} \frac{1}{K}
$$

where $\Delta T_{j}$ denotes the deviation from the natural period $T_{j}$. We choose $K$ equal to the maximum of the first factor. This yields $\max _{\Delta \varphi} H(\Delta \varphi)=1$ as shown in 
Figs. 5.5(a) and (b). Setting $\dot{r}_{j}=0$ in Eq. (5.13a), we obtain

$$
H_{r}(\Delta \varphi)=\frac{1}{K}\left[r(\Delta \varphi)^{2}-\lambda\right],
$$

where $\sqrt{\lambda}$ is experimentally measured as the time average of $r$ over the course of the experiment.

Figure 5.5 shows the two interaction functions $H_{r}$ and $H_{\varphi}$ for the low voltage $V_{0}=1.105 \mathrm{~V}$ in panels (a) and (c) and for the higher voltage $V_{0}=1.2 \mathrm{~V}$ in panels (b) and (d). The (black) curve is a 5th-order Fourier fit from evenly sampled data.

For $V_{0}=1.105 \mathrm{~V}$, the radial interaction function remains approximately constant and the phase interaction function exhibits a sinusoidal shape. Thus, we have $H_{\varphi}(\Delta \varphi)=\sin (\Delta \varphi)$ as considered in Eqs. (6.8).

At this point, we also see a possible cause for the difference between Fig. 5.3(a) and (b). The numerically calculated frequencies coincide with the use of an appropriate phase interaction function. Since we have assumed $\gamma=0$, the radial interaction function does not influence the frequencies, but it does influence the stability of the particular state. The lack of an appropriate radial interaction function might explain the difference between the stability of the states shown in Fig. 5.3(a) and (b).

For $V_{0}=1.2 \mathrm{~V}$, both interaction functions have a more complex structure. In order to take their complex shapes into account in our theory, we approximate $H_{r}$ and $H_{\varphi}$ by Fourier series up to the fifth order. How well this approach works is the topic of the following section. 


\subsection{Relaxation oscillations}

In this section, we present the results for operation at a higher voltage compared to Sec. 5.5, that is, further away from the Hopf bifurcation. We fix the voltage of the system at $V_{0}=1.2 \mathrm{~V}$ such that the electrode current oscillates in a relaxational fashion. We perform experiments similar to the one in Sec. 5.5 with four oscillators coupled in a unidirectional ring, but consider a different range for the time delay.

The oscillators for these experiments now have different intrinsic frequencies compared to Sec. 5.5. For the experiment yielding the results seen in Fig. 5.6 the four oscillators operate at $\omega_{1}=2.421 \pm 0.068 \mathrm{rad} / \mathrm{s}, \omega_{2}=2.445 \pm 0.088 \mathrm{rad} / \mathrm{s}, \omega_{3}=$ $2.407 \pm 0.069 \mathrm{rad} / \mathrm{s}$, and $\omega_{4}=2.449 \pm 0.112 \mathrm{rad} / \mathrm{s}$. During the experiment yielding the results seen in Fig. 5.8 they operate at $\omega_{1}=2.495 \pm 0.109 \mathrm{rad} / \mathrm{s}, \omega_{2}=2.510 \pm 0.124$ $\mathrm{rad} / \mathrm{s}, \omega_{3}=2.448 \pm 0.094 \mathrm{rad} / \mathrm{s}$, and $\omega_{4}=2.515 \pm 0.097 \mathrm{rad} / \mathrm{s}$.

For the chosen voltage, the interaction functions have a more complex shape (cf. Figs. 5.5(b) and (d)) and are approximated by fifth order Fourier series. Therefore, we describe the experiment by the following set of delay-coupled Stuart-Landau equations:

$$
\begin{aligned}
& \dot{r}_{j}=\left(\lambda-r_{j}^{2}\right) r_{j}+K \sum_{n=1}^{N} g_{j n} r_{n}(t-\tau)\left\{\sum_{l=0}^{5} a_{l, r} \cos \left[l\left(\varphi_{n}(t-\tau)-\varphi_{j}\right)\right]+b_{l, r} \sin \left[l\left(\varphi_{n}(t-\tau)-\varphi_{j}\right)\right]\right\}, \\
& \dot{\varphi}_{j}=\omega+K \sum_{n=1}^{N} g_{j n} \frac{r_{n}(t-\tau)}{r_{n}}\left\{\sum_{l=0}^{5} a_{l, \varphi} \cos \left[l\left(\varphi_{n}(t-\tau)-\varphi_{j}\right)\right]+b_{l, \varphi} \sin \left[l\left(\varphi_{n}(t-\tau)-\varphi_{j}\right)\right]\right\},
\end{aligned}
$$

where the Fourier coefficients $a_{l, r}, b_{l, r}, a_{l, \varphi}$, and $b_{l, \varphi}$ are determined by a fit to the experimentally obtained interaction functions $H_{r}$ and $H_{\varphi}$. The coefficients are given in Tab. 6.1. They are normalized such that $\max \left|H_{\varphi}\right|=1$. The coupling strength $K$ 


\begin{tabular}{|ll|}
\hline Radial interaction function & \\
\hline$a_{1, r}=-0.97948$, & $b_{1, r}=-1.82354$ \\
$a_{2, r}=0.36110$, & $b_{2, r}=-0.07963$ \\
$a_{3, r}=0.29724$, & $b_{3, r}=0.54854$ \\
$a_{4, r}=0.05846$, & $b_{4, r}=0.09098$ \\
$a_{5, r}=-0.11558$, & $b_{5, r}=-0.09251$ \\
$a_{0, r}=0.45579$ & \\
\hline \hline Angular interaction function & \\
\hline$a_{1, \varphi}=-0.00610$, & $b_{1, \varphi}=0.31622$ \\
$a_{2, \varphi}=-0.35811$, & $b_{2, \varphi}=0.29020$ \\
$a_{3, \varphi}=-0.25341$, & $b_{3, \varphi}=-0.05585$ \\
$a_{4, \varphi}=-0.13541$, & $b_{4, \varphi}=0.00799$ \\
$a_{5, \varphi}=-0.07183$, & $b_{5, \varphi}=0.00425$ \\
$a_{0, \varphi}=0$ & \\
\hline
\end{tabular}

Table 5.1: Fourier coefficients used in Eqs. (5.16).

still represents the overall coupling strength.

The relaxation oscillators exhibit more complicated cluster and hysteresis behavior as depicted in Figs. 5.6(a) and (b) for experimental and numerical data, respectively. For the latter we use the continuation software DDE-BIFTOOL similar to the case of smooth oscillators. The detected states agree very well with the experimental results in Figs. 5.6(a), where the black arrow marks the starting configuration. Note that only stable solutions relevant to the experimental results are shown. For the comparison between Figs. 5.6(a) and (b), it must also be noted that while the natural frequency $\omega$ varies slowly during the experiments due to drift, it is kept constant in our numerical calculations.

We find a sequence of different cluster states as the time delay $\tau$ is increased or decreased. The primary states discussed earlier in Fig. 5.4, (in-phase, 2-cluster, reverse splay and splay states) are also present in the case of the relaxational oscillators. In the current regime of operation additional qualitatively different states are possible. These secondary states are investigated in the following. 

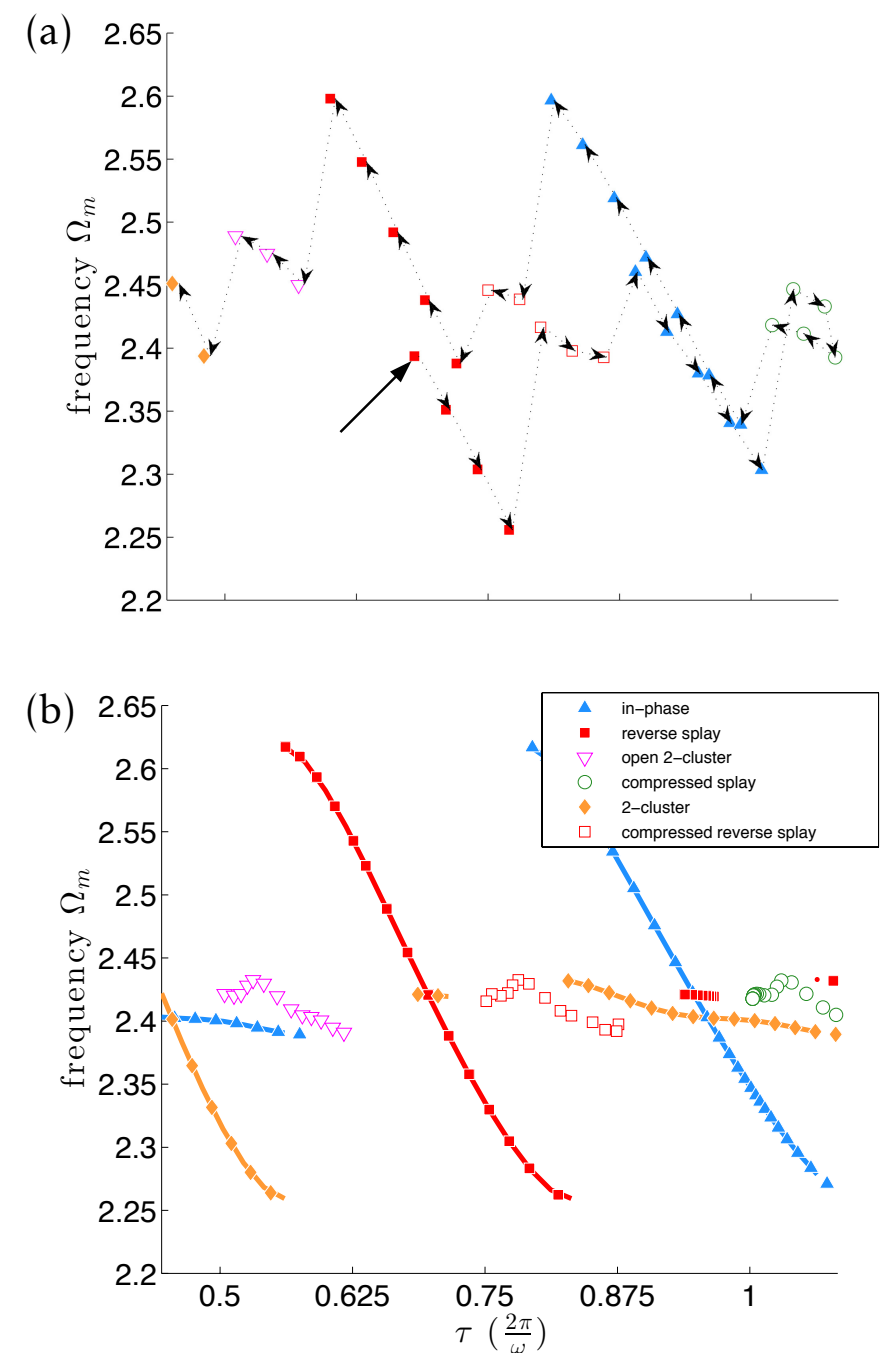

Figure 5.6: Dynamics in dependence on the time delay $\tau$. (a) Experimental data, $V_{0}=1.2 \mathrm{~V}, K=0.10$. The starting state is marked by a black arrow. The increasing and decreasing of $\tau$ during the experiment are shown with arrows. (b) Solutions of Eqs. (5.16) using the continuation tool DDE-BIFTOOL (markers) and numerical solutions of Eqs. (5.4) (lines). In-phase, 2-cluster, compressed splay, reverse splay, compressed reverse splay and open 2cluster states are represented by upward-triangles, diamonds, open circles, squares, open squares and downward-triangles, respectively. The interaction functions $H_{r}$ and $H_{\varphi}$ are chosen as in Eqs. (5.16) and Tab. 6.1. Parameters: $\lambda=2.890, K=0.189$ and $\omega=2.430$. 
The relaxation oscillators still demonstrate hysteresis as $\tau$ is increased and decreased. In a population of smooth oscillators, each cluster state persists over a range of roughly $0.25 \times(2 \pi / \omega)$ of delay after it first occurs, as in Fig. 5.3. With relaxation oscillators, each cluster state persists over a range of roughly $0.125 \times$ $(2 \pi / \omega)$, as in Fig. 5.6(a). The relaxation oscillators tend to alternate between primary and secondary states as $\tau$ is varied. The primary states appear near multiples of $0.25 \times(2 \pi / \omega)$ delay. As $\tau$ is ramped, the phase differences between subsequent elements might vary. As a consequence there could be different phase differences between subsequent elements. This gives rise to the secondary states that appear in between the primary states.
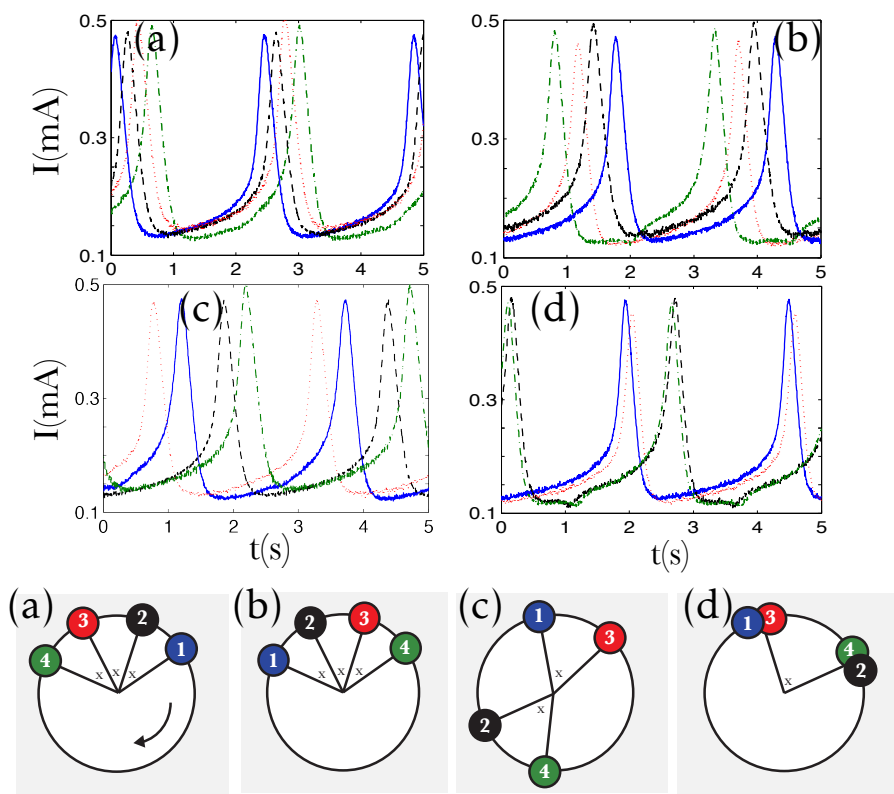

Figure 5.7: Experimental time series (top) and schematic diagram (bottom) for (a) a compressed splay state, (b) reverse compressed splay state, (c) open 2-cluster state, (d) compressed 2-cluster state. For the relaxation oscillators, these are in addition to the possible states seen in Fig. 5.3. Parameters: $V_{0}=1.2 \mathrm{~V}, K=0.10$; time delays: (a) $\tau=1.11 \times 2 \pi / \omega$, (b) $0.82 \times 2 \pi / \omega$, (c) $0.59 \times 2 \pi / \omega$ with $\omega=2.43 \mathrm{rad} / \mathrm{s}$ as in Fig. 5.6 and (d) $0.68 \times 2 \pi / \omega$ with $\omega=2.492 \mathrm{rad} / \mathrm{s}$ as in Fig. 5.8.

For examples of secondary states, Fig. 5.7 shows several experimental time series 
and corresponding schematic diagrams. Figure 5.7(a) depicts a compressed splay state. As in the splay state, we have $\varphi_{4}-\varphi_{3}=\varphi_{3}-\varphi_{2}=\varphi_{2}-\varphi_{1}$, but these phase differences are different from a multiple of $\pi / 2$ (marked by $x$ in the schematic diagram). There also exists a reverse compressed splay state as shown in Fig. 5.7(b). Another secondary state is the open 2-cluster state displayed in Fig. 5.7(c). For this state, the phase differences $\varphi_{3}-\varphi_{1}$ and $\varphi_{4}-\varphi_{2}$ are equal, but the two clusters have a phase lag between them.

As expected from the experiment (cf. Fig. 5.6(a)), the open 2-cluster states are located between the 2-cluster branch of solutions and the reverse splay branch in numerical studies (cf. Fig. 5.6(b)). Likewise, the compressed reverse splay states are located between the reverse splay branch and the in-phase branch and the compressed splay states are located between the in-phase branch and the next splay branch (not shown).

The sequence of cluster states in Fig. 5.6 may seem somewhat arbitrary at first inspection. For example, when $\tau \approx 2.25$ and the branch of compressed reverse splay states becomes unstable, why does the system transition to an in-phase state when a 2-cluster state is also stable at this value of $\tau$ according to Fig. 5.6(b)? When we examine an example time series of the compressed reverse splay state in Fig. 5.7(b), we can see that the phase differences of this state are much closer to those of an in-phase state than to those of a two-cluster state. The experimental states observed depend upon both initial conditions and system parameters, as is typical for systems with multi-stability.

Having the set of primary states in mind, one might ask if there is also a scenario of a compressed 2-cluster state, when $\varphi_{1}=\varphi_{3}$ and $\varphi_{2}=\varphi_{4}$, but the phase differences between the two clusters is no longer equal to $\pi$. Indeed, we find this state as shown in Fig. 5.7(d). The corresponding range of appropriate $\tau$-values is illustrated in 

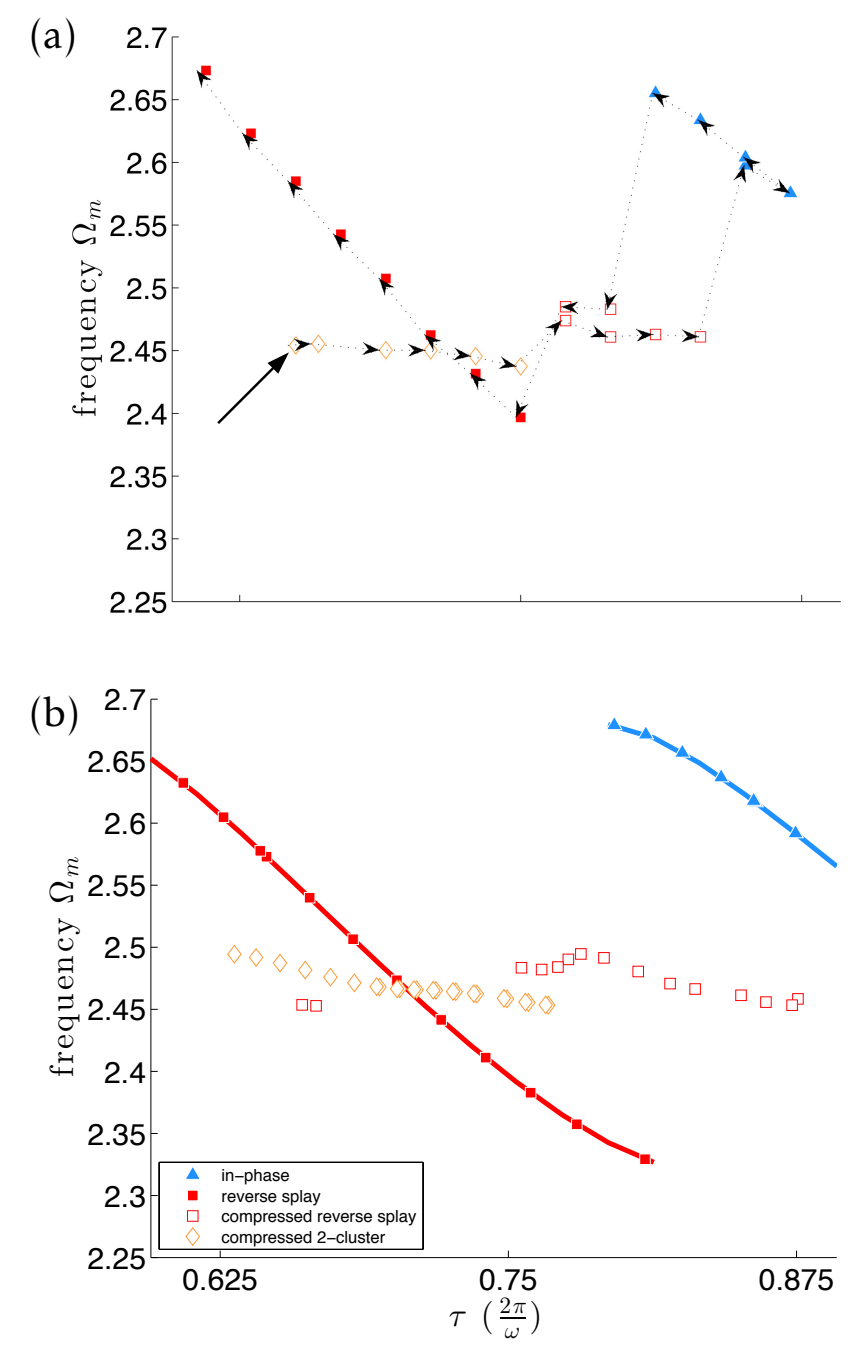

Figure 5.8: Frequency vs. time delay. (a) Experimental data, $V_{0}=1.2 \mathrm{~V}$, $K=0.10$. The starting state is marked by a black arrow. The increasing and decreasing of $\tau$ during the experiment are shown with arrows. (b) $\mathrm{Nu}-$ merical results of Eqs. (5.16) using DDE-BIFTOOL (markers) and solutions of Eqs. (5.4) (lines). The interaction functions $H_{r}$ and $H_{\varphi}$ are chosen as in Eqs. (5.16) and Tab. 6.1. Parameters: $\lambda=2.890, K=0.189$ and $\omega=2.492$.

Fig. 5.8. This figure depicts an experimental measurement for a narrower $\tau$-range and a starting configuration different from Fig. 5.6 (see black arrow) is implemented. This explains why the compressed 2-cluster state was not found in Fig. 5.6 and thus reflects the multi-stability present in the system. Again, only numerical solutions relevant to the states found in the experiment are shown. 


\subsubsection{Extended theory}

The experiments shown in the previous section for the relaxation oscillator motivate an extension of the theory introduced in Sec. 5.3. The extended theory includes the interaction functions with a high order of Fourier coefficients and takes into account the existence of the secondary states. The secondary states can be obtained from Eqs. (5.16) with the Fourier coefficients given in Tab. 6.1, if we assume non-equal radii and phase differences. With the ansatz $r_{j}=$ const. $\equiv r_{0, j}$, and $\varphi_{j}=\Omega t+\Delta \varphi_{j}$, $\Delta \varphi_{j} \in \mathbb{R}$, Eqs. (5.16) yield

$$
\begin{aligned}
0 & =\left(\lambda-r_{0, j}^{2}\right) r_{0, j}+K r_{0, j+1} c_{r}^{j} \\
\Omega & =\omega-\gamma r_{0, j}^{2}+K r_{0, j+1} / r_{0, j} c_{\varphi}^{j}
\end{aligned}
$$

where we used the abbreviation

$$
\begin{aligned}
c_{i}^{j} \equiv \sum_{l=1}^{L}\left\{a_{l, i} \cos \left[l\left(\Delta \varphi_{j+1}-\Delta \varphi_{j}-\Omega \tau\right)\right]\right. \\
\left.+b_{l, i} \sin \left[l\left(\Delta \varphi_{j+1}-\Delta \varphi_{j}-\Omega \tau\right)\right]\right\} .
\end{aligned}
$$

Equation (5.17) is an 8-dimensional ( $\Delta \varphi_{1}=0$ without loss of generality) system of transcendental equations which can be solved numerically. The variational equation reads

$$
\dot{\xi}_{\mathbf{j}}=\mathbf{J}_{j} \xi_{j}+\mathbf{R}_{j} \xi_{\mathbf{j}+\mathbf{1}}(t-\tau)
$$

with the matrices

$$
\mathbf{J}_{j}=\left(\begin{array}{cc}
\lambda-3 r_{0, j}^{2} & -K r_{0, j+1} d_{r}^{j} \\
-2 \gamma r_{0, j}-K \frac{r_{0, j+1}}{r_{0, j}^{2}} c_{\varphi} & -K \frac{r_{0, j+1}}{r_{0, j}} d_{\varphi}^{j}
\end{array}\right)
$$


and

$$
\mathbf{R}_{j}=K\left(\begin{array}{cc}
c_{r}^{j} & r_{0, j+1} d_{r}^{j} \\
c_{\varphi}^{j} / r_{0, j} & r_{0, j+1} / r_{0, j} d_{\varphi}^{j}
\end{array}\right),
$$

using the abbreviation

$$
\begin{aligned}
d_{i}^{j} \equiv \sum_{l=1}^{L}\{ & -a_{l, i} \sin \left[l\left(\Delta \varphi_{j+1}-\Delta \varphi_{j}-\Omega \tau\right)\right] \\
& \left.+b_{l, i} \cos \left[l\left(\Delta \varphi_{j+1}-\Delta \varphi_{j}-\Omega \tau\right)\right]\right\} .
\end{aligned}
$$

Because of the unequal phase differences and radii the variational equation cannot be block-diagonalized (cf. Section 5.3) but the Floquet exponents $\Lambda$ can be obtained from the transcendental equation $\operatorname{det} \mathbf{M}=0$ where the matrix $\mathbf{M}$ has the following form

$$
\mathbf{M}=\left(\begin{array}{cccc}
\mathbf{J}_{1}-\Lambda \mathbf{I}_{2} & \mathbf{R}_{1} e^{-\Lambda \tau} & 0 & 0 \\
0 & \mathbf{J}_{2}-\Lambda \mathbf{I}_{2} & \mathbf{R}_{2} e^{-\Lambda \tau} & 0 \\
0 & 0 & \mathbf{J}_{3}-\Lambda \mathbf{I}_{2} & \mathbf{R}_{3} e^{-\Lambda \tau} \\
\mathbf{R}_{4} e^{-\Lambda \tau} & 0 & 0 & \mathbf{J}_{4}-\Lambda \mathbf{I}_{2}
\end{array}\right)
$$

Solutions of Eq. (5.17) that were found to be stable $(\Lambda<0)$ are plotted as lines in Fig. 5.6(b) and 5.8(b). As expected the lines perfectly agree with the points obtained by the analysis using DDE-BIFTOOL. The disadvantage of this analytic method compared to using the continuation software is that it is difficult to find all solutions of Eq. (5.17); i.e., in Figs. 5.6(b) and 5.8(b) the secondary states were not found analytically but only with the help of DDE-BIFTOOL. However, the analytic method gives further insight into the system, making analysis easier. 


\subsection{Discussion}

We have applied a theoretical analysis of the delay-coupled Stuart-Landau model to a system of chemical oscillators. Experimental results and theory match for cluster synchronization with constant phase lag; the experiment shows an additional type of dynamics with delay-dependent phase lag. These secondary states necessitate a more general approach, and we have extended the model to account for details in the coupling scheme. This lets us approximate the experimental interaction functions and include them in the theoretical model. The numerical results agree with the experimental measurements when the standard Stuart-Landau model is no longer appropriate.

This work illustrates the interplay between theory and experiment; the theory motivates the experiment and the experiment inspires the improvement or extension of the theory. On the one hand, the experiment lets us apply the existing theory. The results for smooth oscillations show that theory accurately predicts the order of cluster transitions in dependence upon the coupling delay and even predicts the frequencies of each state well. On the other hand, we have found a difference in the stability of some states for smooth oscillators. The use of simple sine and cosine functions as interaction functions can no longer be justified, in particular for the radial interaction function.

In experiments with relaxation oscillators, we observe additional secondary cluster states with different phase lags between subsequent clusters. An extended theory matches the experiments. We can now explain the order of transitions between different cluster states first observed in the experiment. The range of stability of each solution branch generally matches. There is still a difference between the exact frequencies of each state, but this is due to the drift in the 
intrinsic frequencies of the chemical oscillators in the experiment.

Our theoretical approach has been shown to produce good results for a system that is not necessarily located near the Hopf bifurcation. This means that the extended model, which permits arbitrary interaction functions, applies to complex non-sinusoidal systems. Although we only consider unidirectional rings, it is interesting to note that the experimental apparatus allows more flexibility in the coupling topology.

\subsection{Acknowledgements}

This work was supported by DFG in the framework of SFB 910, and in part by the National Science Foundation (CBET-0730597). PH acknowledges support by the Federal Ministry of Education and Research (BMBF), Germany (grant no. 01GQ1001B). 


\section{Chapter 6}

\section{Exploring radial dynamics in relaxation oscillators ${ }^{1}$}

\subsection{Notes to the reader}

In Section 6.5.1, we select sets of four oscillators from the whole population of 64; we apply coupling to induce phase locking and vary coupling strength. In Section 6.5.2 we weakly couple pairs of oscillators to obtain coupling functions. Please note that Section 6.3 describing the experimental apparatus is mostly information repeated from Chapter 3 on the experimental apparatus. We repeat this information for the convenience of the reader.

\subsection{Introduction}

Systems of coupled oscillators are of interest in a variety of settings $[41,57]$. Examples include arrays of lasers [15, 90], electronic Josephson junctions [91, 92], the

\footnotetext{
${ }^{1}$ K. A. Blaha, J. Lehnert, A. Keane, P. Hövel, I. Z. Kiss, E. Schöll, J. L. Hudson, “Exploring a phase and radius model for coupled experimental relaxational oscillators" (paper in preparation).
} 
Circadian rhythm [93, 94], and the beating of the heart [95-97], amongst others. Models for such systems can either be constructed from underlying phenomenological behavior, or from observable measurements. In many systems, the first method can be difficult, due to lack of knowledge about the mechanism or difficulty measuring desired quantities, especially in biological systems.

A recent study [69] investigated the clustering of delay-coupled rings of oscillators described by the observable variables of phase and radius (amplitude). The study used experimental oscillators in two regimes: (1) smooth (near-sinusoidal) oscillations near the Hopf bifurcation and (2) relaxational (slow-fast) oscillations far from the Hopf bifurcation. Smooth oscillators were modeled with Hopf normalform oscillators (also called Stuart-Landau oscillators), which are given by a generic Hopf bifurcation model. Modeling relaxation oscillator dynamics required an extended Stuart-Landau model with experimentally-derived functions to describe interactions in both the phase and radius. Phase coupling functions have been shown previously $[33,87]$ in phase-only models such as the Kuramoto phase oscillator model [8]. To our knowledge, the radial interaction function, which describes how the radius of an oscillator varies with coupling, was a first.

In this paper, we explore the conditions where a phase and radius model is needed, and relate the radial interaction function to the underlying voltage stimulation. We present an experiment which is well-described by the phase and radius model that cannot be described by a phase-only model. We present a method for estimating the radial interaction function given knowledge of the coupling parameters, and demonstrate its utility and accuracy.

This paper is organized as follows: In Section 6.3, the experiment using chemical oscillators is described in detail. We present a short summary of relevant results from Ref. [69] in Section 6.4 and introduce the two-variable model. We discuss two 
experimental results in Section 6.5. In Section 6.5.1 we describe an experiment which demonstrates the utility of the two variable model. In Section 6.5.2 we discuss the relationship between stimulation and behavior and use this relationship to predict behavior. Finally, we discuss the general applicability of a phase and radius model in Section 6.6.

\subsection{Experimental setup}

Experiments are performed in an electrochemical cell consisting of $1-\mathrm{mm}$ diameter Ni working electrodes (99.98\%pure), a Pt mesh counter electrode, and $\mathrm{Hg} / \mathrm{Hg}_{2} \mathrm{SO}_{4} / \mathrm{K}_{2} \mathrm{SO}_{4}$ (sat) reference electrode, with a $3 \mathrm{M} \mathrm{H}_{2} \mathrm{SO}_{4}$ electrolyte, shown in Fig. 6.1(a). The cell is enclosed in a jacketed glass vessel maintained at a temperature of $11^{\circ} \mathrm{C}$. An ACM Instruments multi-channel potentiostat is used to set the potentials $V_{0}$ of the electrodes such that they undergo transpassive dissolution. A resistor, $R_{p}=650 \Omega$, is attached to each electrode, causing the dissolution currents $I_{j}$ to oscillate [52]. These resulting electrodissolution currents are measured at $250 \mathrm{~Hz}$ using zero resistance ammeters (ZRAs) attached to a real time data acquisition system.

Oscillators are selected from an array of 64 oscillators. Negligible intrinsic electrical interactions exist between the uncoupled oscillators [98]. The startup or shutdown of an oscillator does not alter the behavior of the other oscillators. The oscillator dynamics has no interdependence when oscillators are functioning in the uncoupled state.

The character of the oscillator may be varied by the choice of applied voltage, $V_{0}$. At low voltages, nearly-sinusoidal oscillations occur while at high voltages, relaxational oscillations occur (see Fig. 6.1(b)). This paper uses relaxation oscillations 

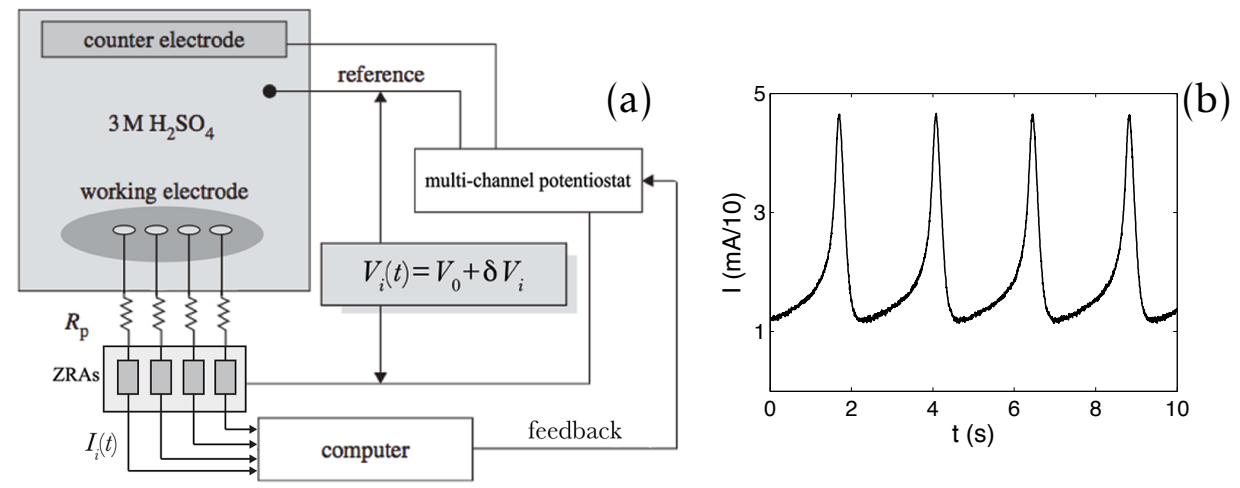

Figure 6.1: (a) Experimental apparatus with multi-channel addressable feedback, $R_{p}$ is the channel resistance of $650 \Omega$. (b) Time series of relaxation oscillators at $V_{0}=1.2 \mathrm{~V}$. ZRA: zero resistance ammeter.

because radial dynamics may be more influential in this regime.

Interactions are introduced using real-time coupling of the form

$$
V_{j}(t)=V_{0}+\delta V_{j}(t)
$$

where $\delta V_{j}$ are the changes in the circuit potentials of the $j$ th elements due to the feedback. The potential drops are given by

$$
x_{j}(t)=V_{j}(t-\tau)-R_{p} \hat{I}_{j}(t-\tau)
$$

where $\hat{I}_{j}$ are the normalized currents measured by the ZRAs. To obtain this quantity, the measurements of the dissolution current $I_{j}$ are first scaled such that the mean value of each channel $\bar{I}_{j}$ is removed as an offset. Then, we perform normalization of the amplitude of the oscillation $I_{j}^{\max }$ relative to the mean amplitude of the electrode 
ensemble $N^{-1} \sum_{n=1}^{N} I_{n}^{\max }$. The feedback voltages are

$$
\delta V_{j}(t)=K \sum_{n=1}^{N} g_{j n} \sum_{s=1}^{S} k_{s} x_{n}\left(t-\tau_{s}\right)^{S},
$$

where $R_{p}=650 \Omega$ is the channel resistance, $K$ is the fixed overall coupling gain and $s$ is the order of coupling. $k_{s}$ and $\tau_{s}$ are the coupling strength and coupling time delay of the sth order of coupling, respectively. $g_{j n}$ is an element of the adjacency matrix $\mathbf{G}$, which describes the structure of the coupling.

In Section 6.5.1, we select four oscillators with similar frequencies for the experiments. We apply unidirectional coupling on a four-member ring with the adjacency matrix

$$
\mathbf{G}=\left(\begin{array}{llll}
0 & 1 & 0 & 0 \\
0 & 0 & 1 & 0 \\
0 & 0 & 0 & 1 \\
1 & 0 & 0 & 0
\end{array}\right) .
$$

In Section 6.5.2, we select two oscillators with dissimilar frequencies for the experiments. We apply coupling on a two-member ring

$$
\mathbf{G}=\left(\begin{array}{ll}
0 & 1 \\
1 & 0
\end{array}\right)
$$

These coupling schemes are implemented via the multi-channel potentiostat (see Fig. 6.1).

We calculate the dynamical variables (amplitudes $r_{j}$ and phases $\varphi_{j}$ ) from the ZRAs' experimental measurement of the electrodissolution current of each oscillator (see Fig. 6.1). From these currents, the phase of each oscillator is found by peak- 
to-peak linear interpolation, where the peak is defined as 0 or $2 \pi$ [87]. From the phases, we can then calculate the average frequencies of the oscillators. The amplitudes are measured as the difference between the peak and the mean value of the electrodissolution current, giving one data point per period.

\subsection{Theory}

In this section we give a short summary of an extended phase and radius model originally developed in Ref. [69] for coupled higher-harmonic oscillators. This method can apply to any topology, but we focus on the unidirectional ring, which we employ in Sec. 6.5. The dynamics of the Stuart-Landau oscillators is given by:

$$
\dot{z}_{j}=f\left(z_{j}\right)+K z_{(j+1) \bmod N}(t-\tau)
$$

with $z_{j}=r_{j} e^{j \varphi_{j}} \in \mathbf{C}, j=1, \ldots, N$, and time delay $\tau$. For notational convenience, we will drop the modulus $N$ in the indices in the following, i.e., $z_{j+1} \equiv z_{(j+1)} \bmod N$.

The local dynamics of each element is given by the normal form of a supercritical Hopf bifurcation:

$$
f(z)=\left[\lambda+i \omega-(1+i \gamma)|z|^{2}\right] z
$$

with real constants $\lambda, \omega \neq 0$, and $\gamma$. This class of systems arises naturally as a generic expansion in center manifold coordinates near a Hopf bifurcation, and therefore its dynamics is generic for many systems close to the Hopf bifurcation. We let $\gamma$ be equal to zero, i.e., the frequency does not depend on the radius.

In polar coordinates with radius and phase variables the system Eq. (6.6) reads 
as follows:

$$
\begin{aligned}
\dot{r}_{j} & =\left(\lambda-r_{j}^{2}\right) r_{j}+K r_{j+1}(t-\tau) \cos \left(\varphi_{j+1}(t-\tau)-\varphi_{j}(t)\right) \\
\dot{\varphi}_{j} & =\omega+K \frac{r_{j+1}(t-\tau)}{r_{j}} \sin \left(\varphi_{j+1}(t-\tau)-\varphi_{j}(t)\right) .
\end{aligned}
$$

We extended this model to describe the behavior of relaxation oscillators with $H_{r}$ and $H_{\varphi}$, the radial and phase interaction functions, respectively:

$$
\begin{aligned}
\dot{r}_{j} & =\left(\lambda-r_{j}^{2}\right) r_{j}+K r_{j+1}(t-\tau) H_{r}\left(\varphi_{j+1}(t-\tau)-\varphi_{j}(t)\right) \\
\dot{\varphi}_{j} & =\omega+K \frac{r_{j+1}(t-\tau)}{r_{j}} H_{\varphi}\left(\varphi_{j+1}(t-\tau)-\varphi_{j}(t)\right) .
\end{aligned}
$$

where $H_{m}$ can be represented by the Fourier series:

$$
\begin{aligned}
c_{m}^{j} \equiv \sum_{l=1}^{L}\left\{a_{l, m} \cos \left[l\left(\Delta \varphi_{j+1}-\Delta \varphi_{j}-\Omega \tau\right)\right]\right. \\
\left.+b_{l, m} \sin \left[l\left(\Delta \varphi_{j+1}-\Delta \varphi_{j}-\Omega \tau\right)\right]\right\} .
\end{aligned}
$$

The parameters $\lambda$ and $\omega$ that belong to the theoretical model can be identified by the dynamics of a single uncoupled oscillator (see Fig. 6.1(b)). $\lambda$ must be set such that the amplitude $r$ is equal to $\sqrt{\lambda}$; while $\omega$ should be chosen such that the period of oscillation, $P$, must be equal to $2 \pi / \omega$. In the experiments $\omega$ is not identical for each oscillator, but the oscillators are chosen so that the values of $\omega$ are very close to each other. As such $\omega$ is simply taken to be the average (i.e. $\omega=N^{-1} \sum_{j=1}^{N} \omega_{j}$ ).

$H_{r}$ and $H_{\varphi}$ are found experimentally by coupling two oscillators such that they 
do not phase lock, and measuring the period and amplitude. Specifically:

$$
\begin{aligned}
H_{r}(\Delta \varphi) & =\frac{1}{K}\left[r(\Delta \varphi)^{2}-\lambda\right] \\
H_{\varphi}(\Delta \varphi) & =\frac{-2 \pi}{K P^{2}}[P(\Delta \varphi)-P] .
\end{aligned}
$$

Fig. 6.2(a) and (b) show $H_{\varphi}$ and $H_{r}$ for a relaxation oscillator held at $V=1.2 \mathrm{~V}$. Further details for $H_{\phi}$ is given in Ref. [33] and for both coupling functions are given in Ref. [69]. The Fourier coefficients for Fig. 6.2 are given in Table 6.1.
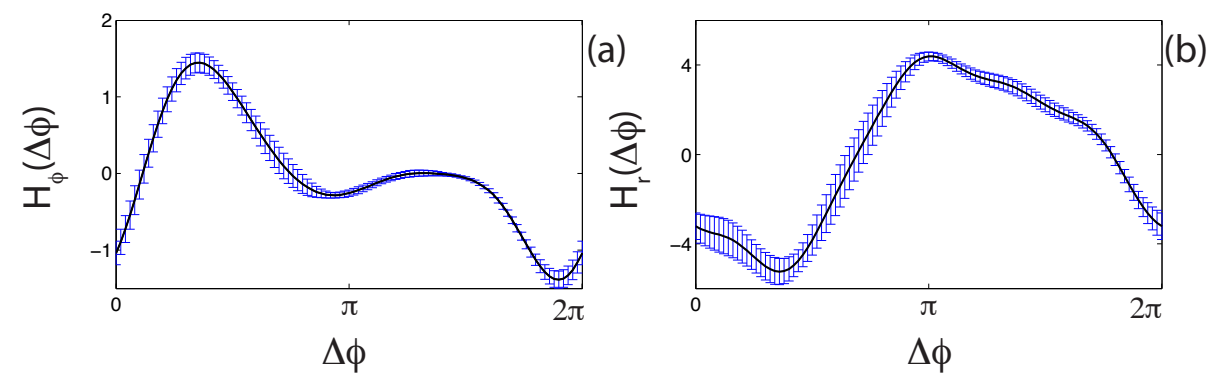

Figure 6.2: (a) Phase interaction function $H_{\varphi}$ and (b) radial interaction function $H_{r}$ for $V_{0}=1.2 \mathrm{~V}$. A 5-term Fourier fit from evenly sampled data is shown by the black curve. Blue error bars show the standard deviations of nine pairs of oscillators. Coefficients for the black curve are given in Table 6.1.

The Kuramoto phase oscillator model is similar to Eq. (6.9a):

$$
\dot{\varphi}_{j}=\omega+K H_{\varphi}\left(\varphi_{j+1}(t-\tau)-\varphi_{j}(t)\right)
$$

$H_{\varphi}$ is the same as $H_{\varphi}$ in the phase and radius model, shown in Fig. 6.2(a).

\subsection{Results}

In Section 6.5.1 we show experimentally that increasing gain causes a cluster transition. We show that the phase-only model cannot predict this result, and that 


\begin{tabular}{|ll|}
\hline Radial interaction function & \\
\hline$a_{1, r}=-4.4645$, & $b_{1, r}=-1.1462$ \\
$a_{2, r}=-0.41226$, & $b_{2, r}=-1.6362$ \\
$a_{3, r}=0.60437$, & $b_{3, r}=0.0655$ \\
$a_{4, r}=0.057681$, & $b_{4, r}=0.58627$ \\
$a_{5, r}=-0.2283$, & $b_{5, r}=0.24678$ \\
\hline \hline Angular interaction function & \\
\hline$a_{1, \varphi}=-0.14137$, & $b_{1, \varphi}=0.75125$ \\
$a_{2, \varphi}=-0.45689$, & $b_{2, \varphi}=0.78142$ \\
$a_{3, \varphi}=-0.24152$, & $b_{3, \varphi}=0.24713$ \\
$a_{4, \varphi}=-0.002844$, & $b_{4, \varphi}=0.12026$ \\
$a_{5, \varphi}=0.02867$, & $b_{5, \varphi}=0.07666$ \\
\hline
\end{tabular}

Table 6.1: Fourier coefficients used in Eqs. (6.9), $H_{\varphi}$ in $\mathrm{rad} / \mathrm{s}, H_{r}$ in $\mathrm{mA} / 10$.

the extended Stuart-Landau model does. In Section 6.5.2 we describe how coupling functions $H_{r}$ and $H_{\varphi}$ may be predicted for systems with nonlinear coupling.

\subsubsection{Cluster transitions due to coupling strength}

Consider an experiment where $\tau$ remains constant and we increment $K$. Before each increment, the system reaches a steady state. If the system phase locks, $d r_{j} / d t$ and $d\left(\varphi_{j+1}-\varphi_{j}\right) / d t=0$ and Eqs. (6.9) become

$$
\begin{aligned}
\frac{r_{j}}{r_{j+1}}\left(r_{j}^{2}-\lambda\right) & =K H_{r}\left(\varphi_{j+1}-\varphi_{j}-\tau\right) \\
\frac{r_{j+2}}{r_{i+1}} H_{\varphi}\left(\varphi_{j+2}-\varphi_{j+1}-\tau\right) & =\frac{r_{j+1}}{r_{j}} H_{\varphi}\left(\varphi_{j+1}-\varphi_{j}-\tau\right) .
\end{aligned}
$$

Eq. (6.13) becomes

$$
H_{\varphi}\left(\varphi_{j+2}-\varphi_{j+1}-\tau\right)=H_{\varphi}\left(\varphi_{j+1}-\varphi_{j}-\tau\right)
$$

Coupling strength cannot influence steady state behavior in Eq. (6.15) because $K$ drops out. 
The coupling strength does affect phase-locking in the phase and radius model in Eqs. (6.14) under certain conditions. If all the phase differences $\varphi_{j+1}-\varphi_{j}$ are equal, then $H_{r}\left(\varphi_{j+1}-\varphi_{j}-\tau\right)$ will be equal for all $j$, and all $r_{j}$ will be equal. Thus, $r_{j+1} / r_{j}$ will be unity. Because there is no other dependence upon $K$, the stability of the system will not be affected by gain.

However, if the phase differences are unequal, as in the case of secondary clusters, introduced in Ref. [69], the radii will be unequal. When this is true, Eq. (6.14a) that the radii will change relative to one another as the coupling gain is changed, even if the phase differences do not. From Fig. 6.2(b), when $\Delta \phi_{j+1, j}-\tau>0, r_{j}<\sqrt{\lambda}$, and if $\Delta \phi_{j+1, j}-\tau<0, r_{j}>\sqrt{\lambda} . r_{j+1} / r_{j}$ sufficiently far from unity may disrupt the cluster state. Additionally, only a certain range of amplitudes are possible in a physical system; an $r_{j}$ outside this range may disrupt the cluster state.

We compare the results of experiment and simulation on a unidirectional fourmember ring when $\tau$ is held constant near 0.6 times the natural period, $P$, and $K$ is incremented. We choose $\tau=0.6 P$ because this delay is likely to induce a secondary cluster state (Fig. 5.7). Fig. 6.3(a) and (b) show the initial secondary cluster state observed in experiment and simulation, respectively.

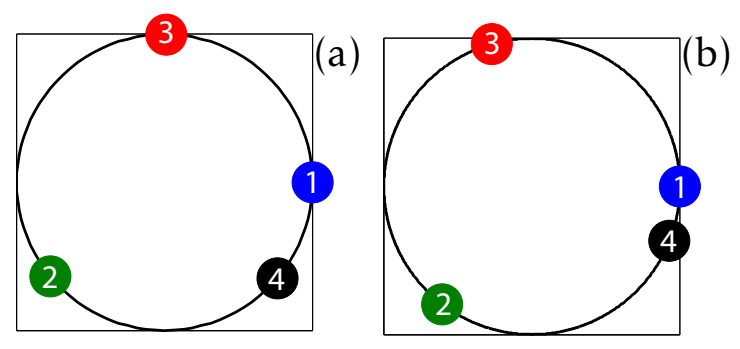

Figure 6.3: The phases, $\varphi_{j}$ as visualized on a phase ring (a) Experimental data for relaxation oscillator, $V=1.2 \mathrm{~V}, K=0.12$. (b) Simulated data using $H_{r}$ and $H_{\varphi}$ as reported in Table. 6.1. $\lambda=6.5, \omega=2.664, \tau=1.5, K=0.5$.

Fig. 6.4 shows the behavior of the radii and phase differences as the coupling 



Figure 6.4: Experimental dynamics for relaxation oscillator, $V=1.20 \mathrm{~V}$, $\tau=0.6 P$. Plots of (a) phase difference, $\varphi_{j}-\varphi_{1}$ and (c) radius in $\mathrm{mA}$. Plots of simulated dynamics (b) phase difference and (d) radius. Simulated with Eq. (6.9 and coefficients in Table 6.1. Phase difference: $\Delta \varphi_{(2,1)}$ in blue squares, $\Delta \varphi_{(3,1)}$ in red triangles, and $\Delta \varphi_{(4,1)}$ in black circles. Radii: 1 in blue circles, 2 in green squares, 3 in red inverted triangles, and 4 in black triangles. $\lambda=6.5, \omega=2.664, \tau=1.5$.

strength, $K$, is incrementally increased in experiments and simulations. Figs. 6.4(a) and (b) show the experimentally observed radii and phase differences, respectively. We start with $K=0.04$ and increment by 0.025 . Around $K=0.06$, the coupling strength is sufficient to induce synchrony, and we observe the secondary cluster state shown in Fig. 6.3(a). This state persists as $K$ is incremented; the phase differences change only slightly, while the radii $r_{i}$ grow or shrink, according to the value of $K H_{r}\left(\varphi_{j+1}-\varphi_{j}-\tau\right)$. At $K=0.1725$, the system switches to a primary cluster state, the reverse splay state. The oscillators in Figs. 6.4(a) and (b) operate at $\omega_{1}=2.901 \pm 0.090$ $\mathrm{rad} / \mathrm{s}, \omega_{2}=2.860 \pm 0.073 \mathrm{rad} / \mathrm{s}, \omega_{3}=2.812 \pm 0.087 \mathrm{rad} / \mathrm{s}$, and $\omega_{4}=2.810 \pm 0.094$ $\mathrm{rad} / \mathrm{s}$ and $r_{1}=2.582 \pm 0.068 \mathrm{~mA} / 10, r_{2}=2.542 \pm 0.066 \mathrm{~mA} / 10, r_{3}=2.546 \pm 0.060$ $\mathrm{mA} / 10$, and $r_{4}=2.580 \pm 0.066 \mathrm{~mA} / 10$. 
Figs. 6.4(c) and (d) show simulated results using the model presented in Eqs. (6.9) and the interaction function coefficients reported in Ref. [previous paper]. Like the experiment, the system begins in a secondary cluster state, shown in Fig. 6.3(b). The phase differences remain constant while the radii change, until a sharp transition occurs at $K=0.5$. In the simulation, the system transitions to a non-realizable state, as negative radii are numerically possible. However, the simulation successfully predicts the loss of stability of the secondary cluster state.

\subsubsection{Predicting the Radial Interaction Function}

In this section, we show that the radial coupling function can be predicted given two pieces of information: (1) the coupling parameters (i.e., $k_{s}$ and $\tau_{s}$ from Eq. (6.3)) and (2) reference coupling functions from other coupling conditions.

First, we perform experiments to obtain the reference coupling functions. We perform experiments with first order (linear) coupling, second order coupling, and third order coupling. We then calculate $H_{r}$ and $H_{\varphi}$ for the three cases. With these three reference coupling functions, we can calculate $H_{r}$ and $H_{\varphi}$ for any $k_{s}$ and $\tau_{s}$ according to:

$$
\begin{aligned}
& H_{r}^{(\text {all })}(\Delta \varphi)=\sum_{s=1}^{S} k_{s} H_{r}^{(s)}\left(\Delta \varphi-\tau_{s}\right) \\
& H_{\varphi}^{(\text {all })}(\Delta \varphi)=\sum_{s=1}^{S} k_{s} H_{\varphi}^{(s)}\left(\Delta \varphi-\tau_{s}\right)
\end{aligned}
$$

Fig. 6.2 and Fig. 6.5 show the reference coupling functions for linear coupling, and second and third order coupling, respectively, calculated from experimental data according to Eqs. (6.11). Each function was calculated from experiments with several pairs of oscillators. Fourier fits were performed on evenly sampled 

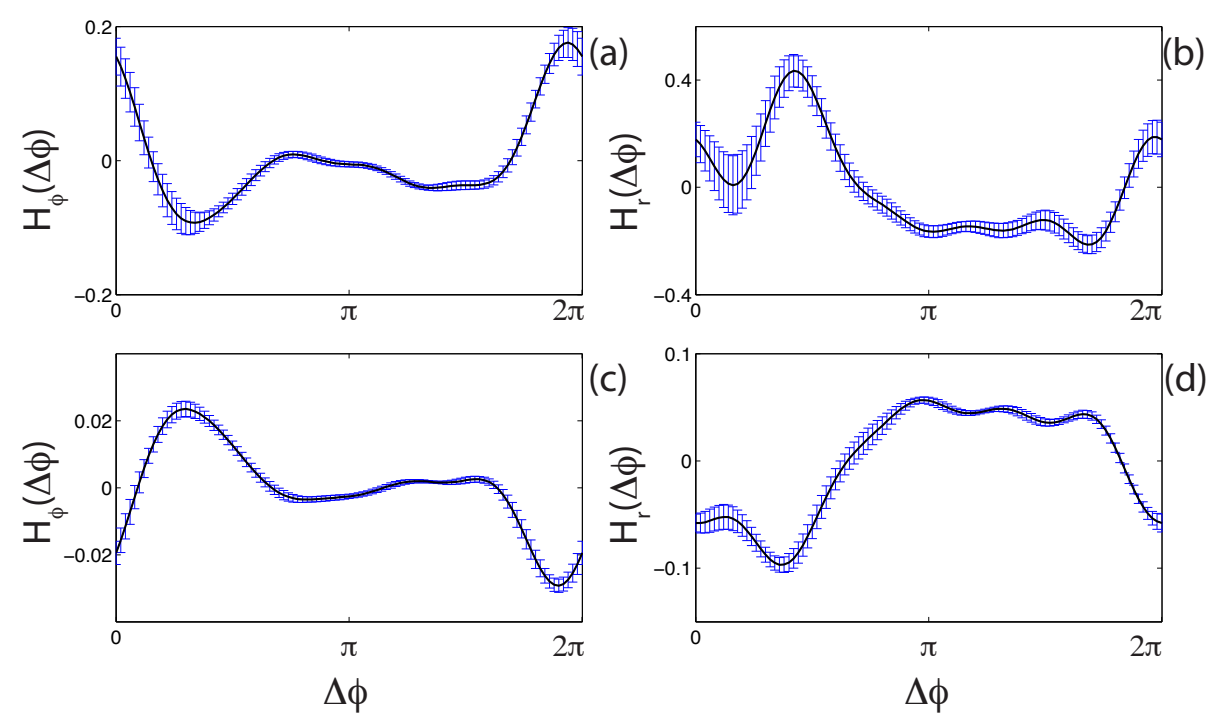

Figure 6.5: Reference coupling functions, $H_{m}^{(s)}$ in black, standard deviations in blue. Second order coupling functions calculated from 11 pairs (a) $H_{\varphi}^{(2)}$ and (b) $H_{r}^{(2)}$. Third order coupling functions calculated from 7 pairs (c) $H_{\varphi}^{(3)}$ and (d) $H_{r}^{(3)}$.

\begin{tabular}{|ll|}
\hline Coupling function errors & \\
\hline$H_{\varphi}^{(1)}=6.50 \%$, & $H_{r}^{(1)}=10.34 \%$ \\
$H_{\varphi}^{(2)}=8.38 \%$, & $H_{r}^{(2)}=6.62 \%$ \\
$H_{\varphi}^{(3)}=6.58 \%$, & $H_{r}^{(3)}=10.40 \%$ \\
\hline
\end{tabular}

Table 6.2: Standard deviations of coupling functions in Figs. 6.2 and 6.5

data. Error was found by the standard deviations of the fits; the average standard deviation for $H_{\varphi}^{(s)}$ was $6.11 \%$ and the average standard deviation for $H_{r}^{(r)}$ was $9.12 \%$. Breakdowns of standard deviation by coupling order are shown in Table 6.2.

With the reference coupling functions, we can predict $H_{m}^{(a l l)}$ for any combination of coupling coefficients. We performed experiments with seven random sets of coupling constants with ten pairs of oscillators. Fig. 6.6 shows the predicted and the experimentally measured coupling functions for one of the pairs at one of the conditions. In $H_{\varphi}$, we can see that the observed coupling function is shifted 

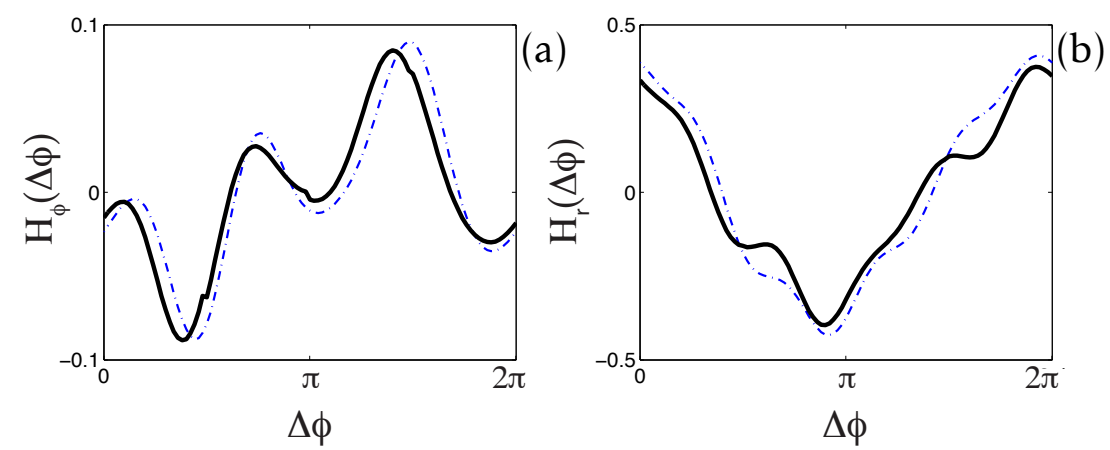

Figure 6.6: Coupling functions, (a) $H_{\varphi}^{(\text {all })}$ and (b) $H_{r}^{(\text {all })}$ with coupling constants $k_{1}=0.03, \tau_{1}=0.5, k_{2}=0.3, \tau_{2}=0.75, k_{3}=3$, and $\tau_{3}=0.25$. Prediction calculated from Eqs. (6.16) shown in black solid, observed coupling function from Eqs. (6.11) shown in blue dot-dash.

slightly compared to the expected one; this is because the frequency naturally drifts upwards, and thus $\tau$ may be larger compared to the period. Such shifts were the primary discrepancy between observation and expectation. From the 70 experiments, there was an average error of $15.4 \%$ for $H_{\varphi}$ and of $15.7 \%$ for $H_{r}$.

\subsection{Discussion}

We modeled the behavior of electrochemical oscillators using the extended StuartLandau model. We showed that by increasing coupling strength, we could cause a unidirectional ring of four oscillators to switch clusters. The modified StuartLandau model also exhibited this transition, which cannot occur in the Kuramoto phase model. We note that the clusters became unstable at different coupling strengths in the experiment and the phase and radius simulation. We suggest that physical systems can only exhibit a certain range of radii; characterizing this range would improve the model.

We described a method for estimating the radial interaction function, if the form of coupling is known as well as the oscillator's response to certain kinds of 
coupling. We believe this is the first time radial behavior has been predicted from knowledge of the stimulation. We showed that interaction functions calculated by this method matched the experimentally-measured interaction functions. Ref. [69] demonstrated that, given knowledge of the interaction function, we can accurately predict clustering on networks of such oscillators. In principle, we are able to engineer the radial interaction function now that we have connected stimulation and behavior. However, changes to the stimulation will affect both the phase and the radius, and both must be taken into account. Interactions with dynamic coupling strength, $K(t)$ or $k_{s}(t)$, might allow one to access radial dynamics specifically, but more study on this topic is required.

We expect the extended phase and radius model described in this paper to be useful for the description of relaxation oscillators. We observed greater radial sensitivity in relaxation than smooth oscillators in the nickel-oxide dissolution system. Generically, oscillators exhibit higher harmonics farther from the Hopf bifurcation $[29,30]$, thus the radial model may aid description of these systems. Additionally, phase-only models have been employed for biological oscillators [31]. We note that many biological oscillators, such as neurons, are highly relaxational with amplitude-sensitive dynamics, and may be better described with a phase-andradius model. Finally, we suggest that the phase and radius model may be a natural extension for systems with moderate coupling that exceeds the "weak coupling" requirement of the Kuramoto phase model [8].

\subsection{Acknowledgments}

This work was supported in part by the National Science Foundation (CBET0730597). 


\section{Chapter 7}

\section{Other investigations}

\subsection{Introduction}

This chapter details several additional projects with coupling networks. Section 7.2 describes experiments and simulations with 64 oscillators with spatially-dependent coupling. Section 7.3 extends the results of Chapter 5 for two additional coupling matrices: (1) a unidirectional ring of 6 oscillators, and (2) a unidirectional ring of 4 oscillators with one weighted value. Section 7.4 describes experiments and simulations with 64 oscillators with two coupled subpopulations.

\subsection{Chimeras}

A chimera is a state of coexisting order and disorder that arises from spatiallydependent coupling [99]. The literature contains few experimental studies with network coupling because such coupling is computationally intense and was not possible until recently. The chimera, which has been studied extensively theoretically [100-102], demonstrates how network coupling can support more complex 


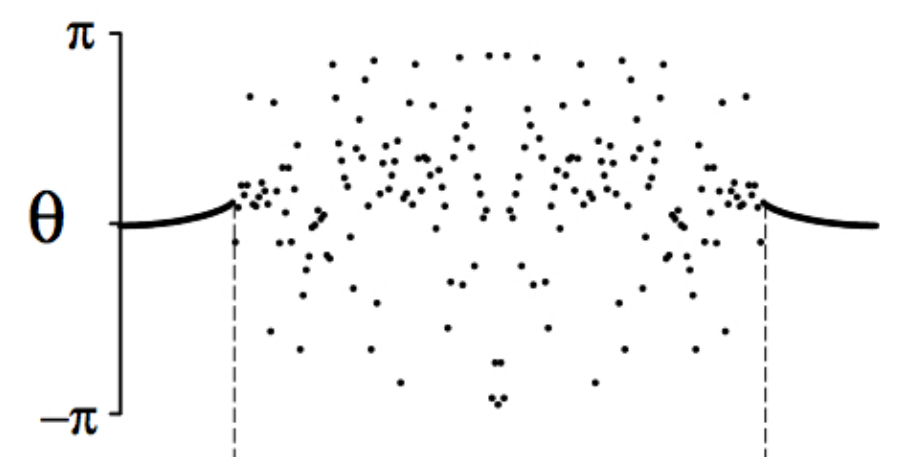

Figure 7.1: An example of the chimera. The phase, $\theta$, as a function of ring position. In the region of order, outside the dashed vertical lines, the oscillators lock in synchrony. In the region of disorder the oscillators fail to synchronize (Fig. 1 from [100]).

behavior than uniform coupling. The chimera state differs fundamentally from synchrony, and cannot be obtained with global coupling.

We investigated the chimera state, shown in Fig. 7.1, with Jörn Davidsen's group at the University of Calgary. Although we successfully produced the chimera in simulations, we could not produce one experimentally. Chimeras cannot exist when noise in a system is too high; we believe our system exceeded that noise threshold. At the time of our investigation, no experiments showing a chimera had been published.

\subsubsection{Theory}

A simple system that can support a chimera state is a population of phase oscillators coupled diffusively on a ring:

$$
\frac{\partial \phi}{\partial t}=\omega+\int_{-\pi}^{\pi} \Gamma\left(x-x^{\prime}\right) \sin \left[\phi\left(x^{\prime}, t\right)-\phi(x, t)-\alpha\right] d x^{\prime}
$$


where $x$ denotes the position of an element on the coupling ring from $-\pi$ to $\pi$, and $\phi(x, t)$ is the phase of the oscillator at position $x$ and time $t . \alpha$ is a phase lag, similar to the previously introduced variable $\tau . \Gamma\left(x-x^{\prime}\right)$ is the coupling vector (analogous to the coupling matrix, $G$ ) which the describes the magnitude of coupling between the oscillator and another oscillator some distance $x-x^{\prime}$ away. In order to support the chimera state, an oscillator is coupled more strongly to neighbors near on the ring than those far away. Any properly-tuned function with coupling that decays with distance can produce a chimera, from an exponential function [99], to a cosine function [100] to a step function [102].

The chimera is only possible with certain coupling vectors and within a range of phase lags. The chimera exists in bistability with a synchronous state, so only some initial conditions successfully produce a chimera. The initial conditions must have a mix of order and disorder, such as the example provided by Abrams and Strogatz [100]:

$$
\phi(x, 0)=6 \exp \left[-30\left(x-\frac{1}{2}\right)^{2}\right] \operatorname{rand}(x),
$$

where rand is a random number.

\subsubsection{Experimental Methods}

We express the equations for the system in more familiar terms

$$
\dot{\phi}_{j}=\omega_{j}+\sum_{n=1}^{N} g_{j, n} \sin \left(\phi_{n}-\phi_{j}-\alpha\right) .
$$


Here $G$ is a coupling graph with a step function. $G$ is obtained from

$$
g_{j, n}^{\prime}= \begin{cases}1, & \text { if } \frac{|j-n|}{N} \leqslant r \\ 0, & \text { if } \frac{|j-n|}{N}>r\end{cases}
$$

where $r$ is a parameter of the coupling matrix giving the width of the step. We calculate the final coupling matrix

$$
g_{j, n}=\frac{g_{j, n}^{\prime}}{\sum_{n} g_{j, n}^{\prime}} .
$$

Thus, $G$ is row-sum 1 and the overall coupling strength is held constant for different values of $r$.

Several obvious limitations exist for the system: we have only 64 oscillators available, the oscillators are naturally slightly heterogeneous, and we have imprecise control over the initial conditions. We induced an initial condition with a mix of order and disorder as an alternative to the initial conditions in Eq. (7.2). To produce this initial condition, $25 \%$ of oscillators were coupled globally amongst themselves, and the remaining oscillators received no coupling. The $25 \%$ synchronize, while the others do not.

We ran simulations with the phase model using the Runge-Kutta in Appendix A.5 to test if a chimera could exist with the conditions described. The existing literature concerned populations of hundreds or thousands of oscillators with little to no heterogeneity. In simulations with 60 slightly heterogeneous phase oscillators, using our initial conditions and a coupling step width of $r=0.7$, we successfully simulated the chimera state. We also produced a chimera state in simulations with Stuart-Landau (phase and radius) oscillators. 
We performed experiments with smooth oscillators using the apparatus described in Fig. 3.3. Experiments were performed in pairs with different initial conditions; the first experiment in the pair was performed immediately following $100 \%$ global coupling, the second experiment was performed immediately following $25 \%$ global coupling. These pairs of experiments were intended to show bistability between a synchronous state and a chimera state. The experiment with the $100 \%$ global coupling should yield a synchronous state, while the partial global coupling should yield a chimera.

\subsubsection{Results and Discussion}

Our experimental results were initially difficult to interpret. As we increased $\tau$, we observed a transition from order to disorder, but not as we expected. Whether or not an element joined the synchronous group was determined solely by frequency, and not ring position. Additionally, we did not observe bistability depending upon the initial conditions. Almost all of the literature on the chimera state explored the behavior of identical oscillators in simulations. We speculated that heterogeneity might cause delocalization of the synchronous group. Simulations with more heterogeneous populations revealed similar delocalization in the synchronous group.

Two possibilities emerged: the chimera was fundamentally changed by heterogeneity, but retained some essential behaviors, or the chimera ceased to exist in the presence of sufficient heterogeneity. Eventually we found a theoretical discussion of the effect of heterogeneity on the chimera. Fig. 7.2 shows the bifurcation diagram, where $D$ is the width of a Lorentzian distribution [103]. We believe that the value of $D$ where the chimera ceases to exist is smaller than the experimental heterogeneity; thus, a chimera cannot be produced on our apparatus. 


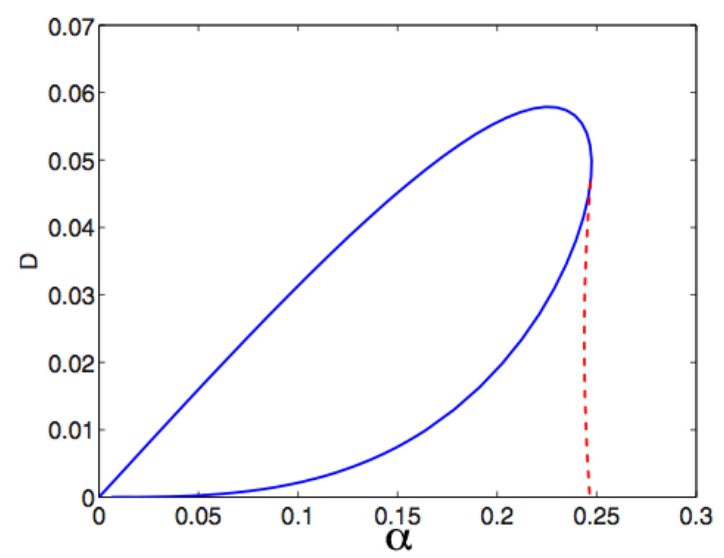

Figure 7.2: The bifurcation diagram for a chimera in the presence of heterogeneity. The blue loop shows where the chimera can exist. When $D$, the heterogeneity, is sufficiently large, the chimera ceases to exist (Fig. 7 from [103]).

Although we failed to produce a chimera state, one interesting and yet unanswered question arose. Some debate exists as to the best form for coupling oscillators. In row-sum zero coupling (as in the Laplacian matrix), oscillator coupling is considered as diffusive coupling $\left(\delta x_{j}(t)=f\left(x_{n}(t)-x_{j}(t)\right)\right)$. In row-sum one coupling (more like the adjacency matrix), oscillator coupling is direct $\left(\delta x_{j}(t)=f\left(x_{n}(t)\right)\right)$. Diffusive coupling is considered to more closely resemble equations for diffusion and energy transfer. The literature lacks consensus on the form of the coupling matrix; some papers use a Laplacian with zero row sum $[47,51]$, some use a matrix with constant row sum [49], and others use an adjacency matrix, which is not necessarily constant row sum [50]. We suggest that diffusive interactions occur as part of the $\mathrm{N}$-dimensional space of the system; the perturbations are already relative to the applied voltage of the original oscillator, and the coupling function may be a function of phase difference whether or not the stimulation is a function of a difference.

In the experiments presented in Chapters 4 and 5, there is qualitatively no difference between diffusive and non-diffusive coupling. In the spatially-dependent 
coupling of this section, diffusive and non-diffusive coupling yield qualitatively different behavior. It is not clear why this system differs.

\subsection{Exploring cluster states on more complex networks}

Chapter 5 explored cluster behavior on a four-member unidirectional ring. This section explores additional network geometries including bigger rings and weighted rings. At present, it is unclear to what extent a cluster state may be tuned by changing only the coupling matrix; further study is required.

\subsubsection{Theory}

The theory for unidirectional rings with more than four members is well-understood for smooth oscillators [49]. We explore the model developed in Chapter 5 for rings of six or more relaxation oscillators, anticipating additional secondary cluster states. Based upon the patterns of the four-member ring, we would expect six primary states (one-cluster, two-cluster, three-cluster, reverse three-cluster, splay, and reverse splay) and six secondary states that represent blends of each two adjacent primary states.

The influence of coupling matrix weighting on phase locking has been shown experimentally in Ref. [25] through the coupling of oscillators with different surface areas. In experiments and simulations, we were able to change the stable cluster states sampled by changing only one value of a unidirectional ring. By changing only one value, we still preserve much of the symmetry originally present in the system, which aids in reducing the complexity of the problem. In a three-member unidirectional ring with one weighted value, the equations for the system are given 
by

$$
\begin{aligned}
& \dot{\phi}_{1}=\omega+g_{1,2} K H\left(\phi_{2}-\phi_{1}-\tau\right) \\
& \dot{\phi}_{2}=\omega+K H\left(\phi_{3}-\phi_{2}-\tau\right) \\
& \dot{\phi}_{3}=\omega+K H\left(\phi_{1}-\phi_{3}-\tau\right) .
\end{aligned}
$$

Thus the stationary states are given by

$$
\begin{aligned}
g_{1,2} H\left(\phi_{2}-\phi_{1}-\tau\right) & =H\left(\phi_{3}-\phi_{2}-\tau\right) \\
H\left(\phi_{3}-\phi_{2}-\tau\right) & =H\left(\phi_{1}-\phi_{3}-\tau\right) .
\end{aligned}
$$

One solution (there can be others) to $H\left(\phi_{3}-\phi_{2}-\tau\right)=H\left(\phi_{1}-\phi_{3}-\tau\right)$ is $\phi_{3}-\phi_{2}-\tau=$ $\phi_{1}-\phi_{3}-\tau$, giving $2 \phi_{3}=\phi_{2}$, if we say that $\phi_{1}=0$ (we are only interested in the phase differences). When we substitute this back into Equation 7.5, we get $g_{1,2} H\left(2 \phi_{3}-\tau\right)=H\left(-\phi_{3}-\tau\right)$. For an $N$-member unidirectional ring with one weighted value, the equation is $g_{1,2} H\left((N-1) \phi_{N}-\tau\right)=H\left(-\phi_{N}-\tau\right)$. With this equation we can plot frequency versus phase difference, and make analytical predictions about the eventual phase differences in a simulation or experiment. Again, there may be other solutions to $H(x)=H(y)$, but this subset of solutions illustrates some of the

system behavior. When $H$ has one maxima such as $H(\Delta \phi)=\sin (\Delta \phi)$, the agreement is especially good.

\subsubsection{Experimental Methods and Results}

We coupled six relaxational oscillators in a unidirectional ring, analogous to the experiments in Chapter 5. As expected, we observed six primary cluster states and six secondary states, shown in Figs. 7.3 and 7.4 respectively. We observe the primary 
states in the following order as coupling delay is increased from zero to $2 \pi$ : one cluster, splay, three cluster, two cluster, reverse three cluster, reverse splay, and one cluster again. The six secondary states are the six blends of adjacent primary states. Additional secondary states will exist, as there will be hybrid states between all pairs of primary states, but we will not observe them in our experiments.
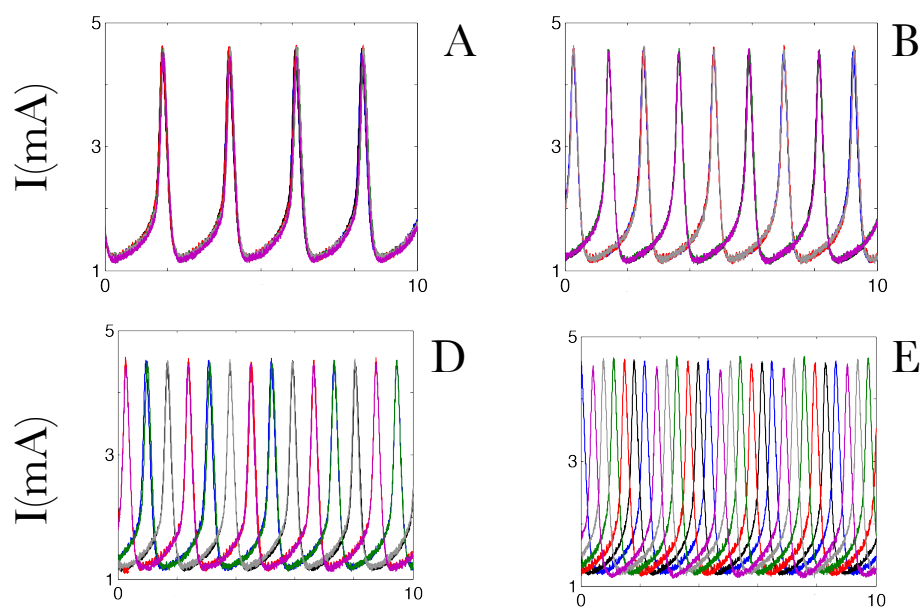

$\mathrm{D}$

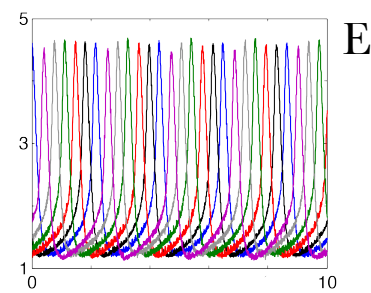

$\mathrm{t}(\mathrm{s})$

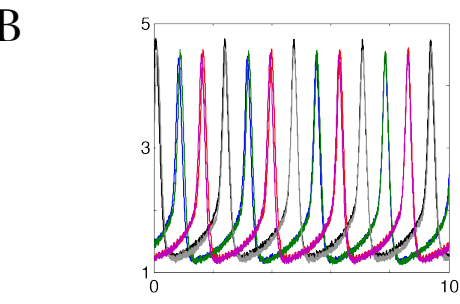

C

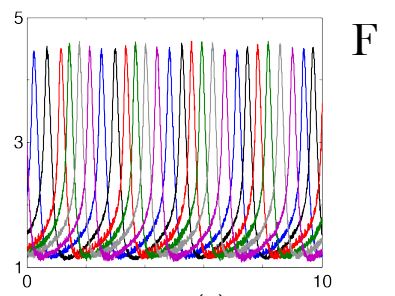

$\mathrm{t}(\mathrm{s})$



Figure 7.3: Time series and schematic on the phase ring of the six primary clusters observed experimentally in a six-member unidirectional ring with delay coupling: (A) the one-cluster state, (B) the two-cluster state, (C) the three-cluster state, (D) the reverse three-cluster state, (E) the splay state, and (F) the reverse splay state. Oscillators are color coded: 1 (blue), 2 (black), 3 (red), 4 (green), 5 (gray), and 6 (purple).

Fig. 7.5 shows the experimental cluster progression as $\tau$ is increased in a ring of six relaxation oscillators. As in the four-member ring, we observe alternation between secondary and primary cluster states. Cluster states are labeled to match the schematics shown in Figs. 7.3 and 7.4.

In experiments with a unidirectional four-member ring of smooth oscillators, 

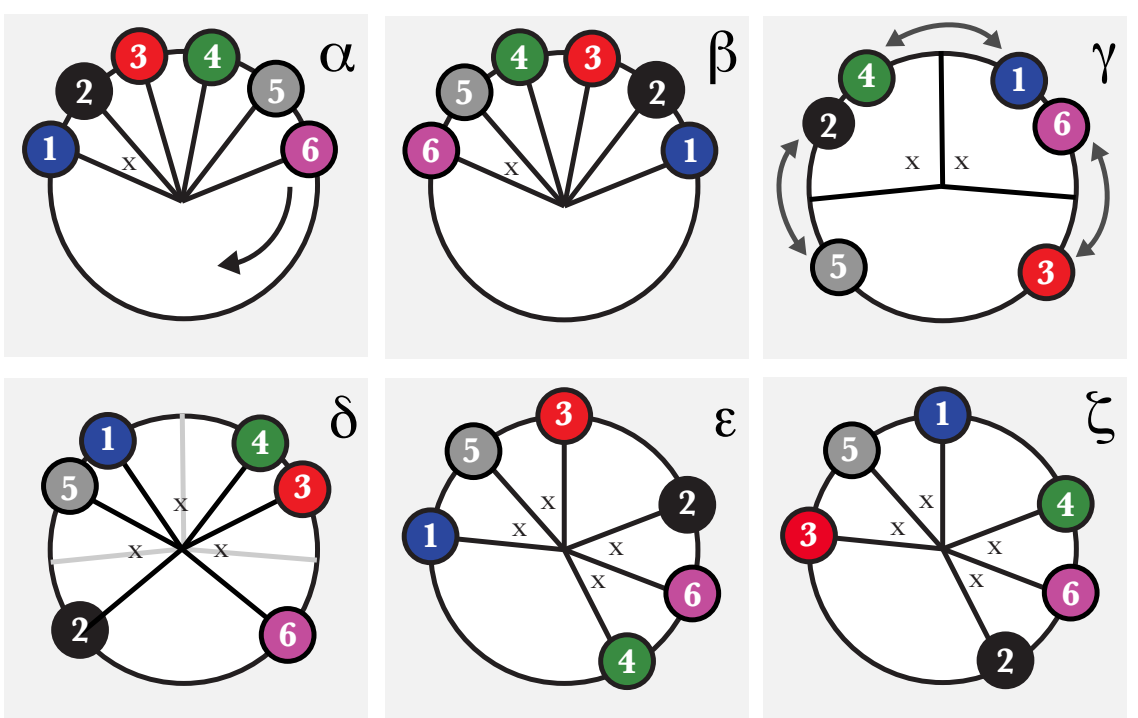

Figure 7.4: Schematic on the phase ring of the six secondary clusters observed experimentally in a six-member unidirectional ring of relaxational oscillators with delay coupling: $(\alpha)$ the compressed splay state, $(\beta)$ the reverse compressed splay state, $(\gamma)$ the splayed three-cluster state, $(\delta)$ the reverse splayed three-cluster state, $(\epsilon)$ the splayed two-cluster state, and $(\zeta)$ the reverse splayed two-cluster state.

we examined the behavior of $g_{1,2}=0.4$ (the coupling strength from oscillator 2 to 1 ). We chose this value because when $g_{1,2}=1 /(N-1)=0.33$ (assuming $\left.H_{\phi}=\Delta \phi\right)$, the system transition from one without hysteresis to one with hysteresis. $g_{1,2}=0.4$ is slightly above this value, and thus exhibits slight hysteresis.

Fig. 7.6(a) and (b) show the cluster frequency depending upon time delay for experiments and phase model simulations $\left(H_{\phi}=\sin (\Delta \phi)\right)$, respectively. Discrepancies between the two are likely because the interaction function for a smooth oscillator is not perfectly sinusoidal. The simulations in Fig. 7.6(b) agree identically with the solutions to $g_{1,2} \sin \left(3 \phi_{4}-\tau\right)=\sin \left(-\phi_{4}-\tau\right)$ from Equations 7.4.

The cluster states that arise from altered values of $g_{1,2}$ with smooth oscillators resemble the compressed splay state found in relaxation oscillators in Chapter 5. We suggest that the secondary clusters in the relaxation oscillator might be due to a 


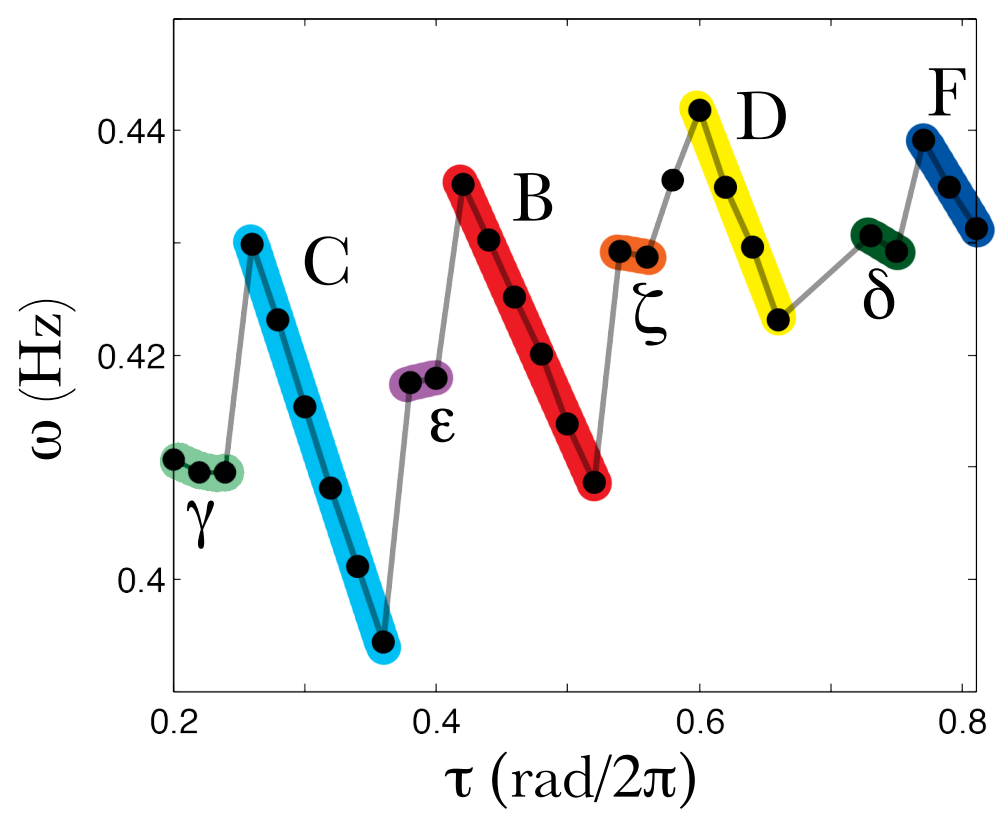

Figure 7.5: The experimental cluster progression on a six-member unidirectional ring as $\tau$ is varied, visualized with the cluster frequency. Oscillators are relaxational, $V=1.2 \mathrm{~V}$. Black dots indicate experiments; colors indicate the cluster configuration. The cluster states occur in the following order: splayed three-cluster (light green, $\gamma$ ), three-cluster (light blue, $C$ ), splayed two-cluster (purple, $\epsilon$ ), two-cluster (red, $B$ ), reverse splayed two-cluster (orange, $\zeta$ ), reverse three-cluster (yellow, $D$ ), reverse splayed three-cluster (dark green, $\delta$ ), reverse splay (dark blue, $F$ ). Letters and greek letters refer to primary and secondary clusters shown in Figs. 7.3 and 7.4, respectively.
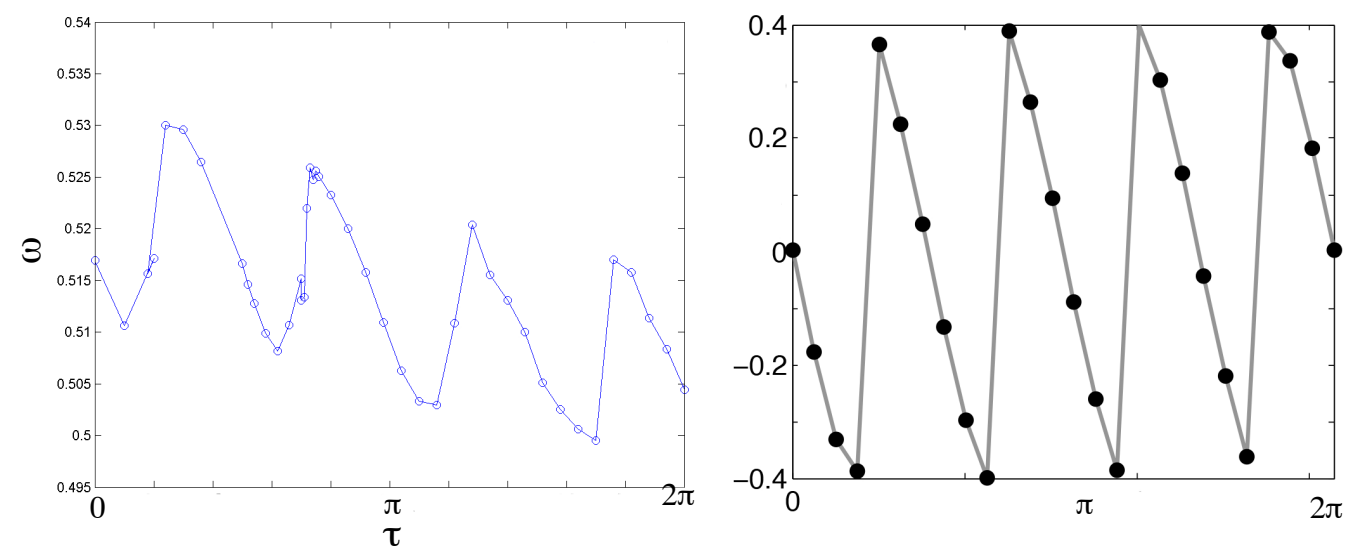

Figure 7.6: Frequency as a function of time delay for a one-value weighted unidirectional ring with $g_{1,2}=0.4$. (Left) Experimental results, (Right) Simulation results with the phase-only model. 
loss of one of the system symmetries. The weighted network described has lost one of its symmetries as well.

\subsection{Radial effects in the conformist/contrarian system}

In the conformist/contrarian system, the total population is split into two subpopulations. The first subpopulation is positively coupled to the mean-field; each element in this population, $j$, experiences pairwise coupling strength $g_{j, n}=K_{1} /(N-1)>0$ from all other oscillators, $N-1$. The second subpopulation is negatively coupled to the mean-field; each element in this population, $j$, experiences pairwise coupling strength $g_{j, n}=K_{2} /(N-1)<0$ from all other oscillators, $N-1$. The schematic and coupling matrix for this system are given in Fig. 7.7(a) and (b), respectively.



A

$$
\left(\begin{array}{cccc}
0 & \kappa_{1} & \kappa_{1} & \kappa_{1} \\
\kappa_{1} & 0 & \kappa_{1} & \kappa_{1} \\
\kappa_{2} & \kappa_{2} & 0 & \kappa_{2} \\
\kappa_{2} & \kappa_{2} & \kappa_{2} & 0
\end{array}\right)^{B}
$$

Figure 7.7: Four elements with conformist/contrarian coupling. Elements 1 and 2 are the conformists, shown in blue, with positive pairwise coupling, $K_{1}>0$. Elements 3 and 4 are the contrarians, shown in pink, with negative pairwise coupling, $K_{2}<0$. (A) A schematic representation. Black arrows indicate positive pairwise coupling, $g_{j, n}>0$; red arrows indicate negative pairwise coupling, $g_{j, n}<0$. (B) Coupling matrix, $G$, corresponding to the schematic in (A). $\kappa_{1}=K_{1} /(N-1)>0, \kappa_{2}=K_{2} /(N-1)<0$.

Oscillators with positive coupling (the conformists) will tend to align with the collective phase, while those with negative coupling (the contrarians) will tend to align opposite to it. When conformists vastly outnumber contrarians, the conformists synchronize and the contrarians phase-lock $\pi$ radians from the conformists. Under other conditions, the system exhibits more complex behavior. 
Studies with interacting subpopulations are relevant for the study of biological systems, which may have many interacting subpopulations.

We performed experiments and simulations with the conformist/contrarian system $[104,105]$. In experiments we systematically observed amplitude differences between the conformists and the contrarians when order emerged in the subpopulations. In simulations with the Stuart-Landau oscillator, we also observed amplitude differences in the organized populations.

\subsubsection{Theory}

The behavior of a population of phase oscillators with a mixture of positive and negative mean field coupling is described by $[104,105]$

$$
\dot{\phi}_{j}^{(s)}=\omega_{j}+\frac{K_{s}}{N} \sum_{n=1}^{N} H\left(\phi_{n}-\phi_{j}^{(s)}\right) .
$$

Here there are two values of $K_{s}$ : the conformists, where $K_{1}>0$, and the contrarians, where $K_{2}<0 . K_{s}$ can be related to the coupling matrix shown in Fig. 7.7B by $K_{s}=\sum_{n} g_{j, n}$. The conformists tend towards the mean-field value as coupling strength is increased, while the contrarians tend to oppose the mean-field value. Hong and Strogatz varied $p$, the percentage of conformists in the population and observed several categories of behavior, and $C[104,105]$. $C$ gives the relative intensity of the conformist coupling, $C=K_{1} /\left(K_{1}-K_{2}\right)$.

At low values of $p$, the contrarians win out and the incoherent state exists, shown in Fig. 7.8(a). A traveling wave state exists at higher values of $p$ when $C$ is $2 / 3$, shown in Fig. 7.8(b). Finally, a $\pi$-state is observed at high values of $p$ and $C$, shown in Fig. 7.8(d). A forth state, the blurred $\pi$-state, is shown in Fig. 7.8(b), but we will not discuss it because it only appears in systems of identical oscillators. 
(a) Incoherent state

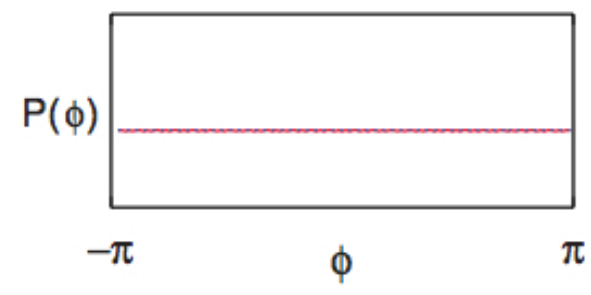

(c) Traveling wave state

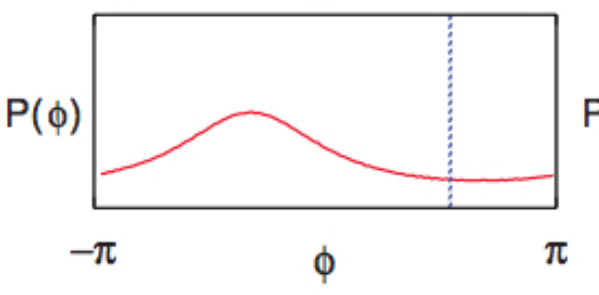

(b) Blurred $\pi$-state

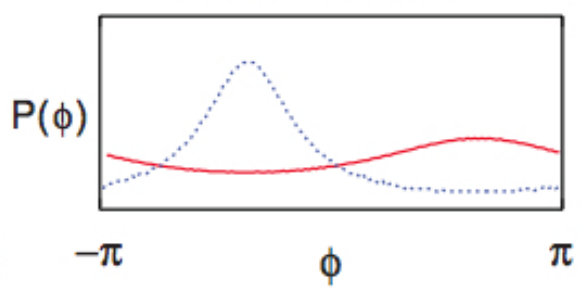

(d) $\pi$-state

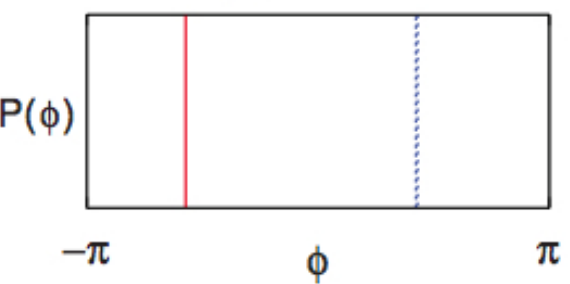

Figure 7.8: States observed in the conformist-contrarian system: (A) the incoherent state $(B)$ the blurred state $(C)$ the traveling wave state and $(D)$ the $\pi$-state [105].

\subsubsection{Experimental Methods}

We used a population of 64 smooth oscillators $(V=1.105 \mathrm{~V})$ with a two-valued coupling matrix using the apparatus in Fig. 3.3. The first $64 p$ oscillators were assigned positive coupling, $K_{1}$. The remaining oscillators were assigned negative coupling, $K_{2}$. We swept through a range of $p$ values with $C=\frac{1}{4}$ or $C=\frac{3}{4}$. We calculated the Kuramoto orders of each population according to

$$
r^{(s)}=\frac{1}{n^{(s)}} \sum_{j=1}^{n^{(s)}} e^{i \phi_{j}^{(s)}} .
$$

We also calculated the mean amplitudes of each population, and the time-dependent phase differences between the two populations. 


\subsubsection{Results and Discussion}

We observed that the oscillator amplitudes depended upon $p$. When $p$ is smaller and the system is disordered, the two populations have similar amplitudes. When $p$ is large enough to induce order, the two populations take on different amplitudes (see Fig. 7.9). These amplitude effects are not possible in a phase-only model, but we have observed them in the Stuart-Landau model:

$$
\begin{aligned}
& \dot{r}_{j}=\left[\lambda-r_{j}^{2}\right] r_{j}+\frac{K^{(s)}}{N} \sum_{n=1}^{N} r_{n} H_{r}\left(\phi_{n}-\phi_{j}\right) \\
& \dot{\phi}_{j}=\omega_{j}+\frac{K^{(s)}}{N} \sum_{n=1}^{N} \frac{r_{n}}{r_{j}} H_{\phi}\left(\phi_{n}-\phi_{j}\right) .
\end{aligned}
$$

This result suggests that the radius still play a role in the dynamics of smooth oscillators, though our findings in Chapter 5 suggest that the effects are less pronounced in a smooth oscillator than a relaxational oscillator. Higher values of gain may significantly change the behavior of the system. The amplitude difference between the conformists and the contrarians show in Fig. 7.9 is about a tenth of that observed in Fig. 6.4(c) ( $\sim 0.005 \mathrm{~mA}$ vs. $\sim 0.05 \mathrm{~mA}$, respectively). Experiments with relaxation oscillators would likely yield more changes to the radius as well. In addition to the amplitude behaviors, we also observed behavior similar to that shown in Fig. 7.8. 


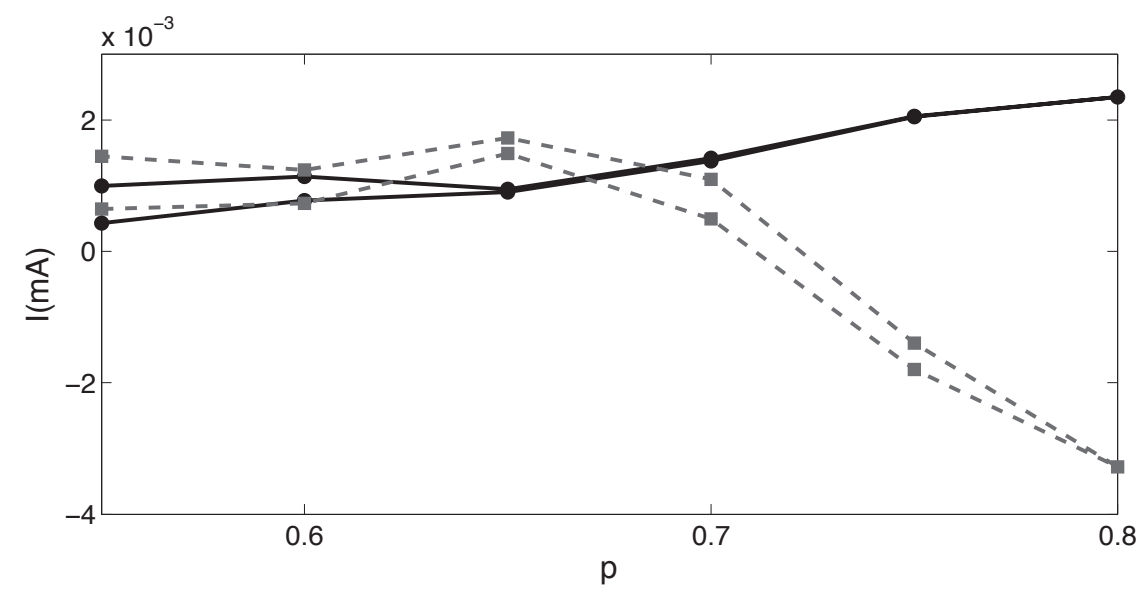

Figure 7.9: The difference in amplitude (from $A_{0}$ ) for conformists (black circles) and for contrarians(gray squares) for smooth experimental oscillators. The populations start becoming ordered near $p=0.7$, at which point we can observe differences in the average amplitudes of each population. $K_{1}=1, K_{2}=-3$, and $C=1 / 4$. 


\section{Chapter 8}

\section{Conclusions and Future Work}

\subsection{Conclusions}

This dissertation explores extensions to the phase model. Such reduced-variable models are a powerful tool already utilized in many settings; these extensions broaden the reach of the model. We specifically consider: (1) phase dynamics of an element as a function of two phases, (2) phase dynamics of a population coupled by a network, and (3) dynamics as a function of both phase and radius. These extended models retain the advantages of the basic phase model; they are still constructed from observed variables and remain easy to work with. Nevertheless, they predict and highlight behaviors not observable in the basic phase model.

Chapter 4 applied a two-phase model and a phase-difference model to experimental data from two coupled oscillators. We showed that the two-phase model recovers coupling directionality, which may be pertinent to biological systems. The two-phase model may also be of particular interest in systems that exhibit learning and dynamic coupling, due to the ability to separate stimulation and response. We showed behavior revealed by the two-phase model but not for the one-phase 
model. We also presented a toy model to demonstrate behavior revealed only by the two-phase model. Prior to our study, the phase-difference model was considered best for similar oscillators; dissimilar oscillators would necessitate a two-phase model. We present exceptions in both directions; the dynamics of the oscillator and the behavior of interest (i.e. phase locking) dictate the appropriateness of the model.

Chapter 5 examined small networks of smooth and relaxation delay-coupled oscillators. The smooth oscillator dynamics, including hysteresis between clusters, were well described by the Stuart-Landau model (a generic model for a Hopf bifurcation). Relaxation oscillator dynamics necessitated extensions to the StuartLandau model; we calculated phase and radial interaction functions describing the dynamics of the phase and radius due to coupling. This is the first radial interaction function described, to our knowledge. The extended model generally matched experimental results; both experiments and the model yielded asymmetrical delaydependent clusters.

Chapter 6 explored the utility of a phase and radius model such as developed in Chapter 5 . We showed that increasing gain will destroy the synchrony of certain cluster states in experiments and the phase and radius model. We presented a method to predict the radial interaction function from the coupling. We suggest that a phase and radius model is preferable to a phase-only model (1) for higher harmonic oscillations and (2) for "non-weak" coupling.

Chapter 7 described several other studies examining complex behavior on network-coupled populations. We explored the chimera state in populations with spatially-dependent coupling. We presented the cluster dynamics in a ring of six delay-coupled experimental oscillators; we identified 12 different clusters. We explored cluster dynamics on small networks with weighting. We described dynamics 
in populations with coupled subpopulations.

\subsection{Future Work}

Although complex systems of oscillators exist in a variety of fields, the greatest future interest likely resides in biological systems. Such systems yield complex emergent behaviors such as breathing, the heartbeat, and human cognition, but they cannot be considered from the scale of an individual element, or often even a single region of an organ. Such a system cannot be handled in its entirety; this suggests the need for a reduced-variable approach. The elements of these systems are individually complex. For example, neurons are highly relaxational and they are sensitive to the strength of stimulation. Biological elements also interact in complex ways; continuing the example, there are multiple kinds of synapses, such as excitatory or inhibitory. Neurons may also communicate electrically or chemically.

Thus, more flexible models are needed, which retain the simplicity of the reduced-variable model yet include essential aspects of the system. Research such as this dissertation on well-behaved test systems is needed to extend and develop models. These models cannot be easily developed solely with biological oscillators; they are more difficult to work with (amongst other things, they can die) and they are noisier.

The above are long term goals of the field. In the more immediate future we present more measured goals to which this dissertation has contributed. If the long term goal is to model biological oscillators, there are several aspects of biological oscillators for which models may need to account.

1. Dynamics for complex coupling matrices. Due to computational limitations, studies of network dynamics are relatively recent; simulations are only recently 
possible for large populations. Our work on small networks in Chapters 5 and 6 serves as a stepping stone toward handling large networks. We also examined several network problems in Chapter 7.

2. Complex and highly nonlinear individual elements. Chapter 6 showed that the phase and radius model described relaxation oscillators better than the existing phase model.

3. Different kinds of coupling. Section 7.4 discussed the conformist/contrarian system, in which some oscillators had positive mean-field coupling and some had negative. This resembles excitatory and inhibitory coupling.

4. Interacting subpopulations. The conformist/contrarian system in Section 7.4 examined the interactions between two populations with positive and negative mean-field coupling.

5. Behaviors other than simple synchrony or systems that evolve over time. The two-phase model in Chapter 4 showed differences between systems not revealed by the basic phase-difference model. This model can also separate stimulation and response, which allows one to see changes in either specifically.

These considerations can also apply to populations of nonbiological oscillators. For example, models with complex coupling networks are also useful for systems of oscillating current generators on the US power grid.

This is the dissertation of the last student in this research group. As such, there are no pending plans for future work from this group. Of course, other groups are still active and we suggest several extensions to the existing work.

1. Chapter 5 explored cluster transitions as the time delay was varied. This study 
can be extended in two dimensions, time delay and coupling gain. Chapter 6 demonstrated that cluster stability is influenced by coupling strength. Especially for relaxation oscillators, we would expect to see changes in the domains of cluster stability change. Based upon the results of Chapter 6, sufficiently strong coupling might cause the system to sample only symmetrical cluster states.

2. The system could be adapted to allow for dynamic coupling strength, $K(t)$ or $K(\varphi)$. For example, from Fig. 6.2, the frequency of a relaxation oscillator is most sensitive to perturbation shortly before and after the peak, while the radius of a relaxation oscillators is most sensitive to perturbation in the trough. We might expect a population more weakly coupled with $\varphi$ near 0 and more strongly coupled with $\varphi$ far from zero would not synchronize.

3. The two phase and the phase and radius approach could be combined, to yield $H_{\phi}\left(\varphi_{i}, \varphi_{j}\right)$ and $H_{r}\left(\varphi_{i}, \varphi_{j}\right)$. If certain oscillations exhibit more radial sensitivity and the two-phase analysis reveals more data, this combination could yield further insights. Our colleagues considered apnea in the neonatal cardiorespiratory system with the two-phase model. Anecdotally, certain distress behaviors were tied to the magnitude of breathing; after an apnea, the infant would often draw a single large breath, while the infant would pant shallowly in other circumstances.

4. A systematic study of possible radii in the system at various voltages would be useful. This could be achieved by applying pulse perturbations to oscillators at various points of their limit cycle with varying strengths of pulses. The possible range of radii would be given by the range of radii achieved by perturbations without destroying the limit cycle. It is not clear how to incorporate 
such information into a model yet. 


\section{Appendix A}

\section{MATLAB Code}

\section{A.1 Time series analysis}

\section{A.1.1 Peak_finder}

$1 \%$

$\%$ This program finds the values of the peaks in data from oscillators, adapted from a program in Craig Rusin's dissertation.

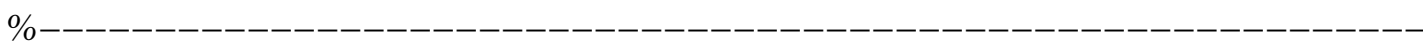

function [ output_args ] = peak_finder(waves, peak_threshold, min_num_pts)

${ }_{6} \%$ row is the length of the data set, col is the number of oscillators.

$[$ row, $\mathrm{col}]=\operatorname{size}($ waves $)$;

indices $=[1: 1:$ row $]$;

for counter $=1: \mathrm{col}$

$11 \%$ selects the vector of data, $y$, for oscillator $=$ counter

$\mathrm{y}=$ waves $(:$, counter $)$; 
$\%$ initializes a matrix for the peaks to be found

$\max \_$data $=[]$;

$\%$ creates a vector of $1 \mathrm{~s}$ and 0 , with $1 \mathrm{~s}$ where the vector exceeds the threshold peak_data_indices $=y>$ peak_threshold;

$\%$ assembles the data exceeding the peak threshold

peak_y = y(peak_data_indices);

$\%$ assembles the indices of the data exceeding the peak threshold

peak_indices $=$ indices $($ peak_data_indices $)$;

$\%$ finds breaks between groups of indices

group_break_indices $=\operatorname{find}(\operatorname{diff}($ peak_indices $)>1)$;

\% for loop loops through all breaks between groups of indices group_counter)];

data_block_y = peak_y(block_indices);

data_block_x = peak_indices(block_indices) ';

$\%$ if the block is longer than the minimum, it is a "window" to be fit for a peak

if length(data_block_y) $>$ min_num_pts 
$\%$ finds the roots of the derivative of the polyfit (to find minima and maxima)

$\mathrm{r}=\operatorname{roots}([\mathrm{p}(1) \star 4 \mathrm{p}(2) \star 3 \mathrm{p}(3) * 2 \mathrm{p}(4)]) ;$

$\%$ finds the real roots lying within the data window

$\%$ if there is only one such root, it is the peak, otherwise, find the one closest to the peak of the raw data

if length $($ max_index $)==1$

else

raw_max index $=$ find $($ data_block_y $==\max ($ data_block_y $))+$ data_block_x

end

$\%$ adds the location of the peak found for this window to the vector of peaks

max_data $=[$ max_data peak_max_location $] ;$

end 
end

61

$\%$ creates a cell for the vector of peaks found found oscillator = counter output_args $\{$ counter $\}=$ max_data;

end

\section{A.1.2 phase_calculator}

$1 \%$

$\%$ This program finds the phase for all data points by peak-to-peak linear interpolation

function phase_data $=$ phase_calculator(peaks)

$6 \%$ finds the number of oscillators in the data

num_elements = length $($ peaks $)$;

$\%$ finds the number of peaks for oscillator 1

start_index = peaks $\{1\}(1)$;

end_index = peaks $\{1\}($ end $)$;

11

$\%$ initializes the phase vector

phase_data = zeros(end_index,num_elements);

current_peak_int $=$ ones(1,num_elements);

16

$\%$ this for loop loops through all the oscillators, osc

for osc $=1$ :num_elements

start_peak = peaks $\{$ osc $\}(1)$;

$\%$ this if statement handles the phases of data before the first peak by 
extrapolating backwards from the first full oscillation

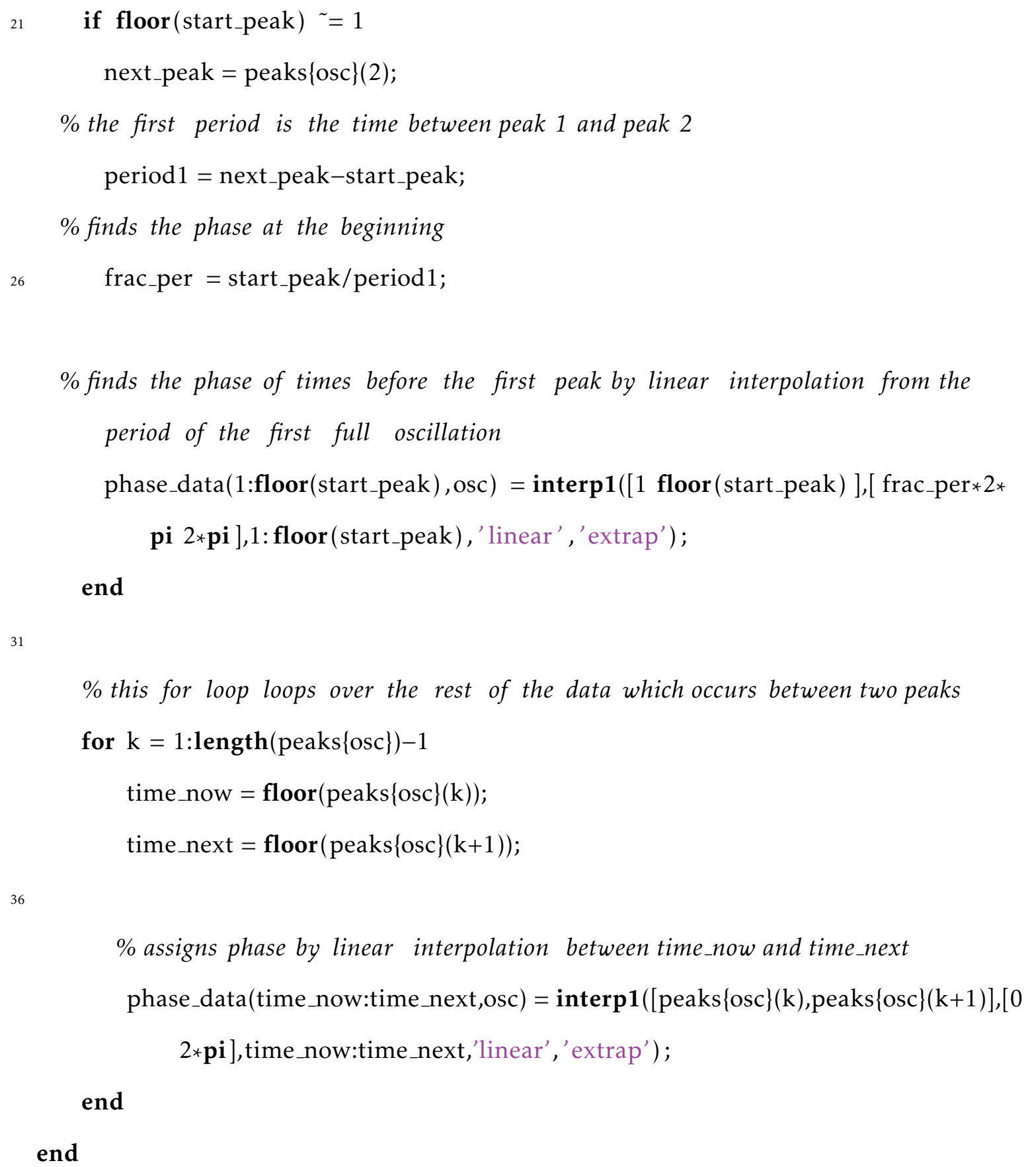




\section{A.2 Fourier analysis}

\section{A.2.1 Program to resample data set: even_sample 2 pi}

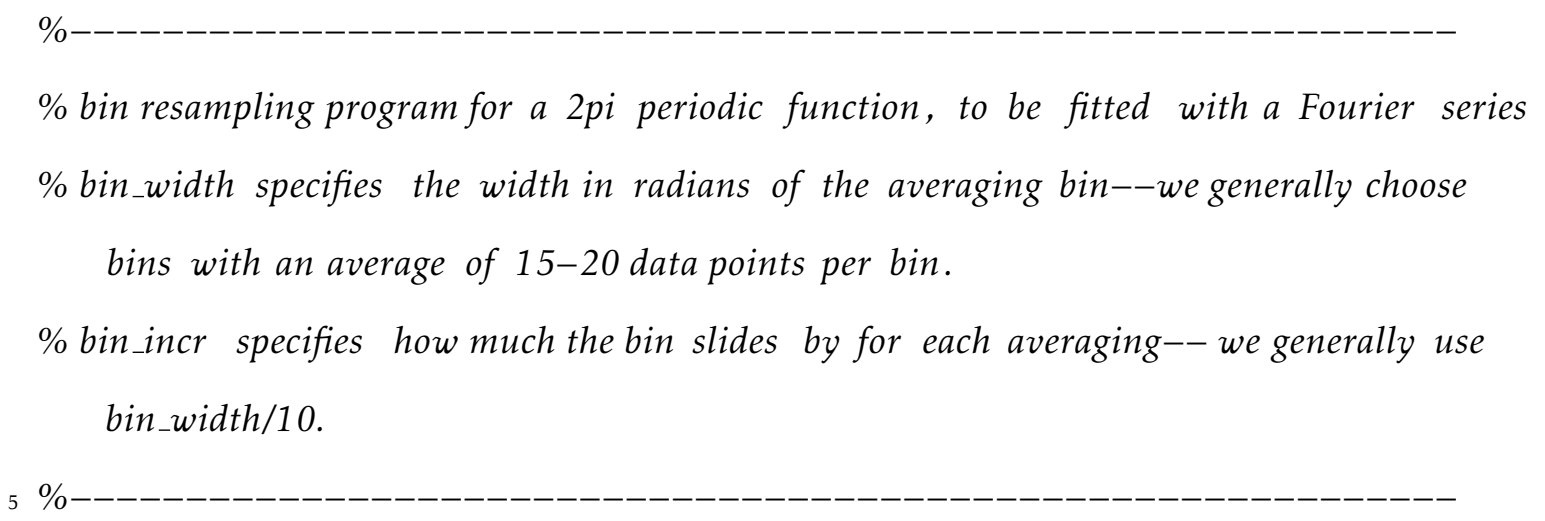

function [resampled_function] = even_sample_2pi(data_in, bin_width, bin_incr)

number_of_bins $=\operatorname{round}(2 * \mathbf{p i} /$ bin_incr $)$;

phase_avg = zeros(size(number_of_bins) $)$;

10 bin_mean $=\operatorname{zeros}(\operatorname{size}($ number_of_bins $))$;

\%below are the vectors giving the left and right hand sides of the phase bin

lil_phi_vector $=(0:(1 /$ number_of_bins $): 1) \times 2 \times \mathbf{p i}-$ bin_width $/ 2$;

big_phi_vector $=(0:(1 /$ number_of_bins $): 1) \times 2 \times \mathbf{p i}+$ bin_width $/ 2$;

15

for $\operatorname{bin}=1$ :number_of_bins

$\%$ defines the beginning and end phases of the current bin

phase_lim_lo = lil_phi_vector (bin);

phase_lim_hi = big_phi_vector(bin);

20

$\%$ defines the middle phase of the bin

phase $\_$avg $($bin $)=($phase_lim_lo+phase_lim_hi $) / 2$;

$\%$ this if statement handles cases that wrap around the 2pi/0 transition 
if phase_lim hi $>2 *$ pi

phase_lo_true $1=$ data_in $(:, 1)>$ phase_lim_lo;

phase_hi_true $1=$ data_in $(:, 1) \quad<=$ phase_lim_hi;

ph_lim_hi_mod $=\bmod ($ phase_lim_hi, $2 \times$ pi $)$;

phase_lo_true2 = data_in $(:, 1)>0$;

phase_hi_true2 = data_in $(:, 1)<=$ ph_lim_hi_mod;

phase_true $1=$ phase_lo_true $1==$ phase_hi_true 1 ;

phase_true $2=$ phase_lo_true $2==$ phase_hi_true2;

phase_true_num = phase_true $1+$ phase_true2;

35

phase_true $=$ logical $($ phase_true_num);

bin $\_$mean $(\operatorname{bin})=$ mean $\left(\right.$ data $\_$in $($phase_true, 2$\left.)\right)$;

elseif phase _lim_lo $<0$

phase_lo_true $1=$ data_in $(:, 1)>$ phase_lim_lo;

phase_hi_true $1=$ data_in $(:, 1)<=$ phase_lim_hi;

ph_lim_lo_mod $=\bmod ($ phase_lim_lo, $2 * \mathbf{p i})$;

phase_lo_true2 = data_in $(:, 1)>$ ph_lim_lo_mod;

45

phase_hi_true $2=$ data_in $(:, 1) \quad<=2 \times$ pi;

phase_true $1=$ phase_lo_true $1==$ phase_hi_true 1 ;

phase_true $2=$ phase_lo_true $2==$ phase_hi_true2;

phase_true_num $=$ phase_true $1+$ phase_true2; 
bin_mean $($ bin $)=$ mean $($ data_in $($ phase_true, 2$))$;

else

\%finds the parts of the input data greater than the minimum phase and less than or equal to the maximum phase phase_lo_true = data_in $(:, 1)>$ phase_lim_lo; phase_hi_true = data_in $(:, 1)<=$ phase_lim_hi; phase_true $=$ phase_lo_true $==$ phase $\_$hi_true; above logic bin $\_$mean $(\operatorname{bin})=$ mean $\left(\right.$ data $\_$in $($phase_true, 2$\left.)\right)$;

end

\section{end}

65

$\%$ outputs two vectors: the middles of the phase bins and the means of the data in those bins

resampled_function $(:, 1)=$ phase_avg;

resampled_function $(:, 2)=$ bin_mean;

\section{A.2.2 Program to find ffts: $\mathrm{fft}$ full}

$2 \%$ This function finds the Fourier coefficients for evenly sampled data $\%$ 
function [ $\mathrm{fft} \_$coeffs , $\left.\mathrm{A} \_0\right]=\mathrm{fft} \_$full $\left(\mathrm{t}, \mathrm{y}, \mathrm{num} \_\right.$coeffs $)$

$\mathrm{L}=(\mathrm{t}($ end $)-\mathrm{t}(1)) / 2$;

7 A_0 $=1 /(2 * \mathrm{~L}) \times$ trap_integrate $(\mathrm{t}, \mathrm{y})$;

fft_coeffs $=$ zeros(num_coeffs, 1$)$;

for $\mathrm{n}=1$ :num_coeffs

A $\_n=1 / L *$ trap_integrate $(t, y * * \cos (n * p i / L * t))$;

$12 \quad \mathrm{~B} \_\mathrm{n}=1 / \mathrm{L} * \operatorname{trap} \_$integrate $(\mathrm{t}, \mathrm{y} * * \sin (\mathrm{n} * \mathrm{pi} / \mathrm{L} * \mathrm{t}))$;

fft_coeffs $(\mathrm{n})=\left[\mathrm{A} \_\mathrm{n}+\mathrm{B} \_\mathrm{n} * 1 \mathrm{i}\right]$;

end

\section{A.2.3 Program to find iffts: inv_fft}

$1 \%$

$\%$ This function generates a function from Fourier coefficients

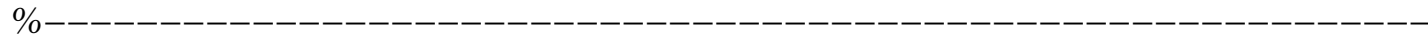

function [invFFT_exp] $=\mathrm{inv} \_f f t\left(\mathrm{fft} \_\right.$coeffs , A_0,t)

${ }_{6} \mathrm{~L}=(\mathrm{t}($ end $)-\mathrm{t}(1)) / 2$;

B_n_sum $=0$;

A_n_sum $=0$;

for $\mathrm{n}=1$ :length ( fft_coeffs )

11

$\mathrm{A} \_\mathrm{n}=\operatorname{real}\left(\mathrm{fft} \_\right.$coeffs $\left.(\mathrm{n})\right)$;

$\mathrm{B} \_\mathrm{n}=\operatorname{imag}\left(\mathrm{fft} \_\right.$coeffs $\left.(\mathrm{n})\right)$; 
$\mathrm{A} \_n \_s u m=A \_n \_s u m+A \_n * \cos (n * \mathbf{p i} * t / L)$;

16 B_n_sum $=$ B_n_sum $+B \_n * \sin (n * p i * t / L)$;

end

invFFT_exp $=$ A_0 + A_n_sum + B_n_sum;

\section{A.3 A sample full analysis of data}

\section{A.3.1 all_calc}

$1 \%$

$\%$ This program calculates phase, radius, and the interaction functions for a day of data

$\%$ For this program, all groups are composed of two oscillators

load_str = 'source_data';

6 folder = 'saving_location';

$\operatorname{mkdir}([$ folder $])$;

$\%$ defines the beginning and end of the data, and how many "groups" are in the data start_run $=0$;

end $\_$run $=6$;

11 num_groups = 10;

for $\mathrm{j}=$ start_run +1 :end_run +1

\%These lines load the labview output

kab_feedback = ['kab_feedback_' num2str(j-1)];

feedback_a $=$ load $([$ kab_feedback '.txt' $])$;

${ }_{16} \%$ reads the defined period and the defined delay. The ratio of the two gives the approximate delay in terms of the period 
K_mat $(j)=$ feedback_a $(1$, num_groups $* 2+19)$;

$\operatorname{delay}(j)=$ feedback_a $(1$, num_groups $* 2+29)$;

tau mat $(j)=$ feedback_a $(1$, num_groups $* 2+29) /$ feedback_a $\left(1\right.$, num $\_$groups $\left.* 2+30\right)$;

csvwrite([folder, ' /K_mat','.csv' ], K_mat)

21 csvwrite([folder,' / delay', ' .csv' ], delay)

\%This "for" loop ramps looks at behavior for each "group" of oscillators

for group=1:num_groups\%num_groups $\% 1: 2$

group_folder $=\left[\right.$ folder,$'$ /group ${ }_{-}^{\prime}$, num 2str(group) $]$;

mkdir(group_folder)

26

\% the Labview program outputs in $m A / 10--h e r e$ we can account for that, if desired

unitize $=0.1$;

\% selects the Labview data for this "group"

feedback $=$ unitize $*$ feedback_a $(:,((2 *($ group -1$))+2):(2 *($ group $)+1))$;

$\%$ filters the data with a 4th order, 129 point Savitsky-Golay filter to remove noise

filt_fb = sgolayfilt (feedback,4,129);

\%This sets a threshold for the peak finder for what qualifies as a peak

threshold $=$ unitize $* 2.5$;

$\%$ Sets the minimum window size which qualifies as a peak

$\min \_$num $=25$;

peaks = peak_finder(filt_fb, threshold, min_num);

$\%$ calculates the phase by linear interpolation

phase_now $=$ phase_calculator $($ peaks $)$;

$\%$ removes the beginning and end of the time series (only 2 seconds), where fitting errors may be present

$41 \quad$ phase $=$ phase_now $(500:$ end $-500,:)$; 
$\%$ finds the minimum length of the two oscillators, so that the peaks can be considered as a matrix

$\min \_$length $=\min ([$ length $(\operatorname{peaks}\{1\})$ length $(\operatorname{peaks}\{2\})]$;

peaks $=[$ peaks $\{1\}(1:$ min_length $) ;$ peaks $\{2\}(1$ :min_length $\}]$;

periods $=\operatorname{diff}($ peaks,1,2)./250;

\% finds the average frequency from the last 25 values of the period

omega $1($ j,group $)=2 \star \mathbf{p i} /$ mean $(\operatorname{periods}(1$, end -25 :end $))$;

omega $2(\mathrm{j}$, group $)=2 \star \mathbf{p i} /$ mean $(\operatorname{periods}(2$, end -25 :end $))$;

$\%$ We find the amplitudes by finding the peaks and subtracting the mean signal of the oscillation.

mean_sig = mean $($ end_fb);

\%to get values for $H_{-}$, first we calculate the amplitudes, the peak value minus the mean value

peak_vals1 = filt_fb $(\operatorname{round}(\operatorname{peaks}(1,:)), 1)$;

peak_vals2 = filt_fb $(\operatorname{round}(\operatorname{peaks}(2,:)), 2)$;

amps $=[$ peak_vals1 - mean_sig $(1) \ldots$

$$
\text { peak_vals2 - mean_sig(2)]; }
$$

$\%$ the scaled radii, the input for the function $H_{-} r$, are found from amp^2$\operatorname{mean}(\operatorname{amp})^{\wedge} 2$

scaled_radius $=\left[\left((\operatorname{amps}(:, 1)) \cdot{ }^{\wedge} 2-(\operatorname{mean}(\operatorname{amps}(:, 1))) \cdot{ }^{\wedge} 2\right) \ldots\right.$

$$
((\operatorname{amps}(:, 2)) \cdot \wedge 2 \quad-\quad(\operatorname{mean}(\operatorname{amps}(:, 2))) \cdot \wedge 2)] ;
$$

\%lambda is the mean of the amplitudes, squared

$\operatorname{lambda} 1(j, g r o u p)=\operatorname{mean}(\operatorname{amps}(:, 1)) . \wedge 2$

$\operatorname{lambda} 2(j, \operatorname{group})=\operatorname{mean}(\operatorname{amps}(:, 2)) .^{\wedge} 2$; 
$\%$ calculates the phase differences

66

phi_delts $=[$ phase_now $(\operatorname{round}(\operatorname{peaks}(1,:)), 2) \ldots$

phase_now $(\operatorname{round}(\operatorname{peaks}(2,:)), 1)]$;

csvwrite([folder, '/omega1','.csv' ], omega1)

csvwrite([folder,' /omega2','.csv' ], omega2)

71

csvwrite([folder, '/lambda1','.csv'], lambda1)

csvwrite([folder, '/lambda2','.csv'], lambda2)

save ([group_folder,' / int_func_-' , num 2str(j-1)], ' phi_delts', ' periods')

save([group_folder,'/rad_int_func,' , num 2str(j-1)], ' phi_delts' , 'radius')

$76 \quad$ end

end

$\%$

$\%$ This section fits the phase data with a Fourier series

$\%$

$81 \%$ The number of Fourier coefficients to find to

num_harms $=5$;

[K_mat $]=\operatorname{csvread}([$ folder,' $/$ K_mat.csv'] $])$;

$[$ delay $]=\operatorname{csvread}([$ folder, ' / delay.csv' $])$;

for group $=1$ :num $\_$groups

86

all_shifts = zeros(num_runs,2);

all_fft_scal = zeros(num_runs,num_harms);

for $\mathrm{j}=$ start_run +1 :end_run +1

$\mathrm{K}=\mathrm{K} \_$mat $(\mathrm{j})$;

load ([ group_folder,'/int_func_' , num 2str(j-1),'.mat'])

91

for osc $=1: 2$ 
$\%$ The pre-interaction functions are vectors of the frequencies and the phase differences

int_pre $=[$ phi_delts $(1:$ end -1, osc $)$ periods $($ osc ,:: ' $]$;

$\%$ The data is sorted according to phase difference

int_func $=$ sortrows $($ int_pre $)$;

96

$\%$ the data is unevenly distributed in dphi, which is incompatible with a Fourier fit.

$\%$ We resample by sliding $a$ bin and finding the mean of the measurements in that bin

data_length $=$ length $($ phi_delts $(:$, osc $))$;

bin_count $=$ round $($ data_length/15);

bin_phase_width $=1 /($ bin_count $) * 2 \times \mathbf{p i}$;

bin_march_size = bin_phase_width/10;

[resamp_if_bin] = even_sample_2pi(int_func, bin_phase_width, bin_march_size); $\mathrm{p}=$ mean $($ resamp_if_bin $(:, 2))$;

\% H_phi scaling according to Miyazaki and Kinoshita, 2006

scale $=-2 * \mathbf{p i} / \mathrm{K} /\left(\mathrm{p}^{\wedge} 2\right)$;

$\%$ The frequency portion is scaled and made zero-mean 
\%calculates the shifts needed to account for the applied coupling

116

$[\mathrm{fft}, \mathrm{A}]=\mathrm{fft} \_f u l l($ resamp_if $(:, 1)$, resamp_shift,num_harms);

121

all_fft $($ osc $, \mathrm{j},:)=\mathbf{f f t}$;

csvwrite([group_folder,'/ fft_coeffs1_all ' ,'.csv'], all_fft1 )

all_shifts $(j$, osc $)=$ shift_amt 1 ;

csvwrite([group_folder,'/ all_shifts ' ,'.csv'], all_shifts )

126

end

end

end

end

$\%$

$131 \%$ This section fits the radial data with a Fourier series

$\%$

$[$ K_mat $]=\operatorname{csvread}([$ folder,' $/$ K_mat.csv' $])$;

$[$ delay $]=\operatorname{csvread}([$ folder, ' / delay.csv' $])$;

for group=1:num_groups

136

all_rad_fft1_scal = zeros(num_runs,num_harms);

all_rad_fft2_scal = zeros(num_runs,num_harms);

[ all_shifts ] = csvread $\left(\left[\operatorname{group} \_f o l d e r, ' /\right.\right.$ all_shifts .csv'] $)$;

for $\mathrm{j}=$ start_run +1 :end_run +1

$\mathrm{K}=\mathrm{K} \_$mat $(\mathrm{j})$;

141

load ([group_folder,' /rad_int_func_' , num 2str(j-1),'.mat']) 




146

data_length $=$ length $($ phi_delts $(:$, osc $))$;

bin_count $=$ round $($ data_length/15);

bin_phase_width $=1 /($ bin_count $) * 2 * \mathbf{p i}$;

bin_march_size = bin_phase_width/10;

151

[resamp_if $]=$ even_sample_2pi(rad_int_func, bin_phase_width,

bin_march_size);

resamp_shift $=$ circshift $($ resamp_if $(:, 2),-$ all_shifts $(j$, osc $))$;

scaler $=\mathrm{K}$;

156

$\left[\operatorname{rad} \_f f t, A\right]=f f t \_f u l l($ resamp_if $(:, 1)$, resamp_shift,num_harms);

$\operatorname{rad} \_f f t=\operatorname{rad} \_f f t . /$ scaler;

$\mathrm{A}=\mathrm{A} /$ scaler;

rad_all_fft $($ osc $, \mathrm{j},:)=$ rad_fft ;

161

end

csvwrite([group_folder,'/ rad_fft_coeffs_all ' ,'.csv'], rad_all_fft )

end

end 


\section{A.4 Preparing a coupling matrix for labview}

\section{A.4.1 Create_Labview_MTX_File}



11 function Create_Labview_MTX_File(varargin)

string_mtx_size="' ;

switch(length(varargin))

$16 \quad$ case $\{1\}$

MTX = varargin $\{1\} ;$

$\%$ allows the user to graphically select the file path

[1File ,1Path] = uiputfile(' *.txt' ,'Please Select File ... ');

if $($ lFile $==0)$

$21 \quad \mathrm{OK}=0$;

else

[pathstr, name,ext $]=$ fileparts $($ lFile $)$;

$[$ name $]=$ fileparts $($ File $)$; 


\section{if (isempty (ext))}

26

$$
\text { ext=' .txt'; }
$$

end

filename $=$ fullfile $(1$ Path,$[$ name ext $])$;

$$
\mathrm{OK}=1 \text {; }
$$

end

$31 \quad$ case $\{2\}$

filename = varargin $\{1\}$;

MTX = varargin $\{2\}$;

if (isnumeric(MTX) \& ischar(filename))

$\mathrm{OK}=1$;

${ }_{36}$ end

$\%$ if there was no error on the kinds of data input

if $(\mathrm{OK})$

if (test_MTX(MTX))

41

$\operatorname{disp}([$ 'File: ' filename' was written successfully'])

else

mtX_size=size $($ MTX);

for $(i=1$ :length(mtx_size $)-1)$

string_mtx_size $=\left[\right.$ string_mtx_size num $2 \operatorname{str}\left(m t x \_s i z e(i)\right)$ ' $\left.x^{\prime}\right]$;

46

end

$\%$ displays an error if the matrix is not $64 \times 64$

string_mtx_size $=\left[\right.$ string_mtx_size num $\left.2 \operatorname{str}\left(m t x \_s i z e\left(l e n g t h\left(m t x \_s i z e\right)\right)\right)\right]$;

$\operatorname{disp}([$ 'ERROR: The given data matrix is ' string_mtx_size '. It must be 64 $\left.\left.\mathrm{x} 64^{\prime}\right]\right)$

end 


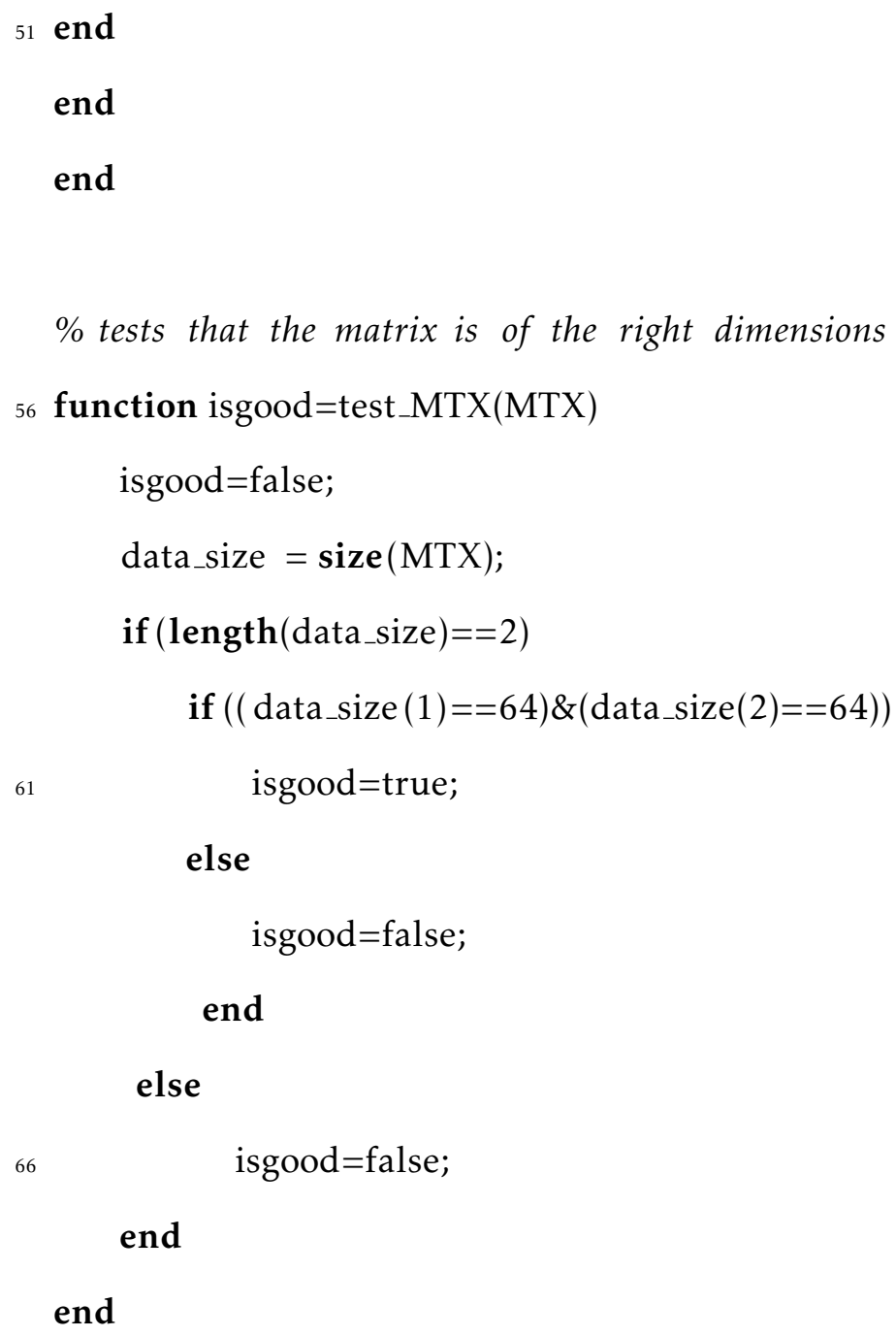

${ }_{71} \%$ tells matlab how to write out the file with tab-delimiting for labview function cgr_dlmwrite(filename,MTX_data)

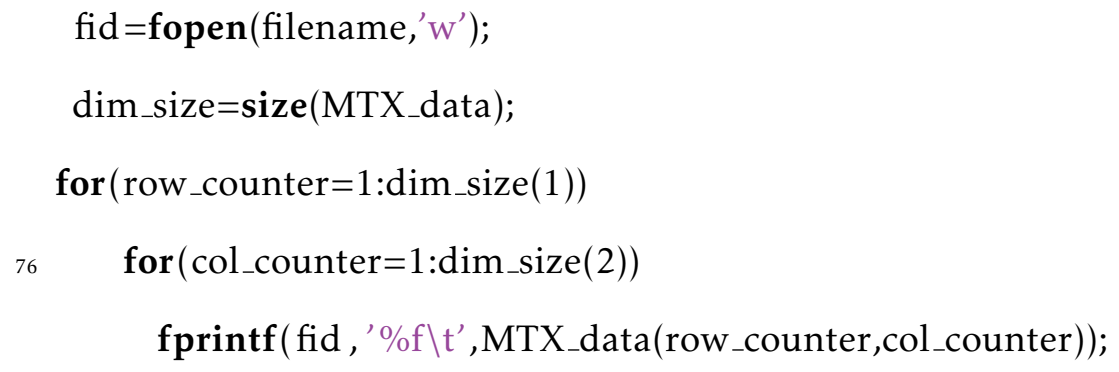


end

fprintf(fid ,'\n');

end

${ }_{81}$ fclose(fid);

end

\section{A.5 Simulation}

\section{A.5.1 A sample simulator}

$\%$ This program calculates phi_j and radius_j with the extended Stuart-Landau model when delay is steadily increased for oscillators with interaction functions expressed in terms of Fourier coefficients

$3 \%$ After the first iteration with random conditions, the simulator uses the end conditions of the previous simulation as the new starting conditions.

\%The Runge-Kutta used is contained at the end of this program. It allows for delay by including a vector from the previous simulation.

\%The function outputs tau (delay), OMG (the collective frequency), ph_delts (the phase differences), and r_end (the steady-state radii). Inputs are $K$ (gain), iter (the calculation iteration), and folder (where the data is stored).

$\%$

8 function [tau,OMG,ph_delts,r_end] = rad_phi_and_r_v_tau(tau,K,iter,folder)

\%var_set contains the variables loaded in for this calculation (the frequency, the Fourier coefficients, the base amplitude, and the coupling matrix) var_set 
$13 \%$ the anonymous functions define phase and radius with time for the simulator fun $\_$ph $=@\left(r_{-}, p h \_, r_{-} t a u, p h \_t a u, t\right.$, coup_row, num_osc,omega_set,K)...

(omega_set $+\ldots$

$\mathrm{K} * \operatorname{sum}\left(\right.$ coup_row_*r_tau. $/\left(\mathrm{r}_{-} *\right.$ ones $(1$, num_osc $\left.)\right) *\left(\mathrm{f} \operatorname{cor}(1) * \cos \left(\mathrm{ph} \_t a u-p h \_\right)+\mathrm{fcoi}(1) * \sin \right.$ (ph_tau-ph_)...

18

$+\mathrm{f} \operatorname{cor}(2) * \cos \left(2 *\left(\mathrm{ph}_{-} \mathrm{tau}-\mathrm{ph} \_\right)\right)+\mathrm{f} \operatorname{coi}(2) * \sin \left(2 *\left(\mathrm{ph}_{-} \mathrm{tau}-\mathrm{ph}{ }_{-}\right)\right.$ )... $+\mathrm{f} \operatorname{cor}(3) * \cos \left(3 *\left(\mathrm{ph}_{-} \mathrm{tau}-\mathrm{ph} \mathrm{f}_{-}\right)\right)+\mathrm{f} \operatorname{coi}(3) * \sin \left(3 *\left(\mathrm{ph}_{-} \mathrm{tau}-\mathrm{ph}_{-}\right)\right.$ )...

+ fcor $(4) * \cos \left(4 *\left(\right.\right.$ ph_tau $\left.\left._{-} \mathrm{ph}_{-}\right)\right)+\mathrm{f} \operatorname{coi}(4) * \sin \left(4 *\left(\right.\right.$ ph_tau $\left._{-} \mathrm{ph}_{-}\right)$ )...

$+\mathrm{f} \operatorname{cor}(5) * \cos (5 *($ ph_tau - ph_ $))+\mathrm{f} \operatorname{coi}(5) * \sin (5 *($ ph_tau - ph_ $)$ ))));\%

fun $\_r=@\left(r_{-}, p h \_, r \_t a u, p h \_t a u, t, c o u p \_r o w\right.$, num_osc,omega_set,K)...

$\mathrm{K} * \operatorname{sum}\left(\right.$ coup_row $* \mathrm{r}_{-}$tau $*\left(\mathrm{r} 0+\operatorname{rcor}(1) * \cos \left(\mathrm{ph}_{-} \mathrm{tau}-\mathrm{ph}_{-}\right)+\operatorname{rcoi}(1) * \sin \left(\mathrm{ph} \_t a u-\mathrm{ph}_{-}\right) \ldots\right.$

$+\operatorname{rcor}(2) * \cos (2 *($ ph_tau - ph_ $))+\operatorname{rcoi}(2) * \sin (2 *($ ph_tau - ph_ $)$ )...

$+\operatorname{rcor}(3) * \cos \left(3 *\left(\mathrm{ph}_{-} \mathrm{tau}-\mathrm{ph}_{-}\right)\right)+\operatorname{rcoi}(3) * \sin \left(3 *\left(\mathrm{ph}_{-} \mathrm{tau}-\mathrm{ph}_{-}\right)\right.$ )$\ldots$

$+\operatorname{rcor}(4) * \cos \left(4 *\left(\mathrm{ph} \_t a u_{-}-\mathrm{ph}_{-}\right)\right)+\operatorname{rcoi}(4) * \sin \left(4 *\left(\mathrm{ph}_{-}\right.\right.$tau $\left.-\mathrm{ph}_{-}\right)$ )... 
$\%$ initializing the time, phase, and radius vectors

$\operatorname{tmax}=200 ;$

$\mathrm{h}=0.005$;

$33 \quad$ t_sol $=[0: \mathrm{h}: \mathrm{tmax}]^{\prime}$;

ph_in $=\operatorname{zeros}\left(\right.$ length $\left.\left(\mathrm{t} \_s o l\right), N\right)$;

$\mathrm{r} \_$in $=\operatorname{zeros}\left(\right.$ length $\left(\mathrm{t}_{-}\right.$sol $\left.), \mathrm{N}\right)$;

\%If this is the first simulation: Assigns random phases and the base radius sqrt( lambda)

38 if iter $==1$

ph_start $=2 * \operatorname{pi} \times \operatorname{rand}(1, N)$;

r_start = sqrt (lam);

ph_out_all = [];

43

r_out_all = [];

ph_in $(1,:)=$ ph_start;

r_in $(1,:)=$ r_start ;

$\%$ If this is not the first iteration: Reads in the conditions of the last iteration previously written to file

else

48

[ph_out_all $]=\operatorname{csvread}([$ folder, ' / ph_out_all.csv'] $)$;

ph_in $(1,:)=$ ph_out_all $($ end,$:)$;

[r_out_all ] = csvread $([$ folder, ' / r_out_all .csv' $])$;

r_in $(1,:)=$ r_out_all (end ,:);

$53 \quad$ end 
$\%$ the main simulation. The first iteration uses $K=0$, to initialize the system [r_out,ph_out] = rk_2var(fun_r,fun_ph,t_sol ,r_in ,ph_in, h,omega,K,coup_mtx,tau); ph_end $=\bmod ($ ph_out $($ end,$:), 2 *$ pi $)$; ph_out_all = [ph_out_all; ph_end $]$; csvwrite([folder,' / ph_out_all' ,' .csv' ], ph_out_all)

r_end $=$ mean $\left(r_{-}\right.$out $($end $-500:$ end,$\left.:)\right)$;

r_out_all = [r_out_all ; r_end $]$;

$\%$ finds the mean frequency toward the end, when the system has presumably reached a steady state

$\mathrm{OMG}=\operatorname{mean}(\operatorname{diff}($ ph_out $($ end-2000:end-1000,1) $) / \mathrm{h})$;

$\%$ writes out the phase and radii

csvwrite([folder, ' / phi ', 'num2str(iter), '. csv' ], ph_out)

csvwrite([folder, '/r_out_' , num 2str(iter), '. csv' ], r_out)

$$
\begin{aligned}
& \operatorname{ph} \_d e l t s(1)=\text { mean }\left(\operatorname{delt} \_v e c s(1, \text { end-4999:end })\right) \\
& \text { ph_delts }(2)=\text { mean }(\text { delt_vecs }(2, \text { end-4999:end }))
\end{aligned}
$$


ph_delts(3) = mean(delt_vecs(3,end-4999:end));

ph_delts(4) = mean(delt_vecs(4,end-4999:end));

83

r_end

end

$\%$

${ }_{88} \%$ The Runge-Kutta

$\%$

function [sol_A,sol_B] = rk_2var(fun_a,fun_b,t,A_, B_, h,omega,K,coup_mtx,tau)

tau_steps = round $(\operatorname{tau} / \mathrm{h})$;

93 num_osc = length(omega);

for $\mathrm{i}=1$ :length $\left(\mathrm{A}_{-}\right)$

if tau $==0 \%$ if tau is zero, no time delay compensation

A_tau $=$ A_(i ,: ) ;

B_tau $=$ B_$_{-}(\mathrm{i},:)$;

$98 \quad$ else

$$
\begin{aligned}
& \text { A_tau }=\text { A_(i }- \text { tau_steps },:) \\
& \text { B_tau }=\text { B_(i }- \text { tau_steps },:) ;
\end{aligned}
$$

end

end

103

for $\mathrm{N}=1$ :num_osc

$\mathrm{k} 11(\mathrm{~N})=\mathrm{h} *$ fun_a(A_(i,N), B_(i,N), A_tau, B_tau, $\mathrm{t}(\mathrm{i})$, coup_mtx $(\mathrm{N}, \mathrm{i})$, num_osc, $\operatorname{omega}(\mathrm{N}), \mathrm{K})$; 
$\mathrm{k} 12(\mathrm{~N})=\mathrm{h} \times$ fun_b(A_(i,N), B_(i,N), A_tau, B_tau, $\mathrm{t}(\mathrm{i})$, coup_mtx $(\mathrm{N}, \mathrm{i})$, num_osc, $\operatorname{omega}(\mathrm{N}), \mathrm{K})$;

108

$\mathrm{k} 21(\mathrm{~N})=\mathrm{h} * \mathrm{fun} \_\mathrm{a}\left(\mathrm{A}_{-}(\mathrm{i}, \mathrm{N})+0.5 * \mathrm{k} 11(\mathrm{~N}), \mathrm{B}_{-}(\mathrm{i}, \mathrm{N})+0.5 * \mathrm{k} 12(\mathrm{~N}), \mathrm{A}_{-} \operatorname{tau}+0.5 * \mathrm{k} 11(\mathrm{~N})\right.$,

B_tau $+0.5 * k 12(\mathrm{~N}), \mathrm{t}(\mathrm{i})+0.5 * \mathrm{~h}$, coup_mtx $(\mathrm{N},:)$, num_osc,omega $(\mathrm{N}), \mathrm{K})$;

$\mathrm{k} 22(\mathrm{~N})=\mathrm{h} * \mathrm{fun} \_\mathrm{b}\left(\mathrm{A}_{-}(\mathrm{i}, \mathrm{N})+0.5 * \mathrm{k} 11(\mathrm{~N}), \mathrm{B}_{-}(\mathrm{i}, \mathrm{N})+0.5 * \mathrm{k} 12(\mathrm{~N}), \mathrm{A}_{-} \mathrm{tau}+0.5 * \mathrm{k} 11(\mathrm{~N})\right.$,

B_tau $+0.5 * k 12(\mathrm{~N}), \mathrm{t}(\mathrm{i})+0.5 * \mathrm{~h}$, coup_mtx $(\mathrm{N}, \mathrm{:})$, num_osc,omega $(\mathrm{N}), \mathrm{K})$;

$\mathrm{k} 31(\mathrm{~N})=\mathrm{h} *$ fun $\_\mathrm{a}\left(\mathrm{A}_{-}(\mathrm{i}, \mathrm{N})+0.5 * \mathrm{k} 21(\mathrm{~N}), \mathrm{B}_{-}(\mathrm{i}, \mathrm{N})+0.5 * \mathrm{k} 22(\mathrm{~N}), \mathrm{A}_{-} \operatorname{tau}+0.5 * \mathrm{k} 21(\mathrm{~N})\right.$,

B_tau $+0.5 * k 22(\mathrm{~N}), \mathrm{t}(\mathrm{i})+0.5 * \mathrm{~h}$, coup $\_\mathrm{mtx}(\mathrm{N},:)$, num_osc,omega $\left.(\mathrm{N}), \mathrm{K}\right)$;

113

$\mathrm{k} 32(\mathrm{~N})=\mathrm{h} * \mathrm{fun} \_\mathrm{b}\left(\mathrm{A}_{-}(\mathrm{i}, \mathrm{N})+0.5 * \mathrm{k} 21(\mathrm{~N}), \mathrm{B}_{-}(\mathrm{i}, \mathrm{N})+0.5 * \mathrm{k} 22(\mathrm{~N}), \mathrm{A}_{-} \mathrm{tau}+0.5 * \mathrm{k} 21(\mathrm{~N})\right.$,

B_tau $+0.5 * k 22(\mathrm{~N}), \mathrm{t}(\mathrm{i})+0.5 * h$, coup_mtx $(\mathrm{N},:)$, num_osc,omega $(\mathrm{N}), \mathrm{K})$;

$\mathrm{k} 41(\mathrm{~N})=\mathrm{h} * \mathrm{fun} \_\mathrm{a}\left(\mathrm{A}_{-}(\mathrm{i}, \mathrm{N})+\mathrm{k} 31(\mathrm{~N}), \mathrm{B}_{-}(\mathrm{i}, \mathrm{N})+0.5 * \mathrm{k} 32(\mathrm{~N}), \mathrm{A}_{-}\right.$tau $+\mathrm{k} 31(\mathrm{~N}), \mathrm{B}_{-}$tau +

k32(N), t (i ) + h, coup_mtx $(\mathrm{N},:)$, num_osc,omega $(\mathrm{N}), \mathrm{K})$;

$\mathrm{k} 42(\mathrm{~N})=\mathrm{h} * \mathrm{fun} \_\mathrm{b}\left(\mathrm{A}_{-}(\mathrm{i}, \mathrm{N})+\mathrm{k} 31(\mathrm{~N}), \mathrm{B}_{-}(\mathrm{i}, \mathrm{N})+0.5 * \mathrm{k} 32(\mathrm{~N}), \mathrm{A}_{-}\right.$tau $+\mathrm{k} 31(\mathrm{~N}), \mathrm{B}_{-}$tau + k32(N), t(i)+ h, coup_mtx $(\mathrm{N},:)$, num_osc,omega $(\mathrm{N}), \mathrm{K})$;

118

$A_{-}(\mathrm{i}+1, N)=A_{-}(\mathrm{i}, \mathrm{N})+1 / 6 *(\mathrm{k} 11(\mathrm{~N})+2 * \mathrm{k} 21(\mathrm{~N})+2 * \mathrm{k} 31(\mathrm{~N})+\mathrm{k} 41(\mathrm{~N}))+\mathrm{h}^{\wedge} 5$;

$\mathrm{B}_{-}(\mathrm{i}+1, \mathrm{~N})=\mathrm{B}_{-}(\mathrm{i}, \mathrm{N})+1 / 6 *(\mathrm{k} 12(\mathrm{~N})+2 * \mathrm{k} 22(\mathrm{~N})+2 * \mathrm{k} 32(\mathrm{~N})+\mathrm{k} 42(\mathrm{~N}))+\mathrm{h}^{\wedge} 5$;

\footnotetext{
end

123 end

sol_A = A_(1:end-1,:);

sol_B = B_(1:end-1,:);
} 
end 


\section{Bibliography}

[1] A. M. Yacomotti, G. B. Mindlin, M. Giudici, S. Balle, S. Barland, and J. Tredicce. Coupled optical excitable cells. Phys. Rev. E, 66(3):036227, 2002. doi: 10. 1103/PhysRevE.66.036227.

[2] B. Blasius, A. Huppert, and L. Stone. Complex dynamics and phase synchronization in spatially extended ecological systems. Nature, 399:354-359, 1999.

[3] J. Miyazaki and S. Kinoshita. Determination of a coupling function in multicoupled oscillators. Phys. Rev. Lett., 96:194101, 2006.

[4] K. Weisenfeld, P. Colet, and S.H. Strogatz. Frequency locking in josephson arrays: Connection with the kuramoto model. Physical Review E, 57:15631569, 1998.

[5] M. G. Rosenblum, L. Cimponeriu, A. Bezerianos, A. Patzak, and R. Mrowka. Identification of coupling direction: Application to cardiorespiratory interaction. Phys. Rev. E, 65(4, Part 1), 2002.

[6] R. Mrowka, L. Cimponeriu, A. Patzak, and M. G. Rosenblum. Directionality of coupling of physiological subsystems: age-related changes of cardiorespiratory interaction during different sleep stages in babies. Am. 
J. Physiol. Regul. Integr. Comp. Physiol., 285(6):R1395-R1401, 2003. doi: 10.1152/ajpregu.00373.2003.

[7] A. T. Winfree. Biological rhythms and the behavior of populations of coupled oscillators. J. Theor. Biol., 16:15, 1967.

[8] Y. Kuramoto. Chemical Oscillations, Waves and Turbulence. Springer, Berlin, 1984.

[9] H. Sakaguchi, S. Shinomoto, and Y. Kuramoto. Local and global selfentrainments in oscillator lattices. Prog. Theor. Phys., 77(5):1005-1010, 1987.

[10] G. B. Ermentrout and N. Kopell. Multiple pulse interactions and averaging in systems of coupled neural oscillators. J. Math. Biol., 29(3):195-217, 1991.

[11] J. A. Acebron, L. L. Bonilla, C. J. Perez Vicente, F. Ritort, and R. Spigler. The Kuramoto model: A simple paradigm for synchronization phenomena. Rev. Mod. Phys., 77(1):137-175, 2005.

[12] K. Okuda. Variety and generality of clustering in globally coupled oscillators. Physica D, 63(3-4):424-436, 1993.

[13] P. Seliger, S. C. Young, and L. S. Tsimring. Plasticity and learning in a network of coupled phase oscillators. Phys. Rev. E, 61:041906, 2002.

[14] H. Sompolinsky, D. Golomb, and D. Kleinfeld. Cooperative dynamics in visual processing. Phys. Rev. A, 43:6990, 1991.

[15] D. J. DeShazer, R. Breban, E. Ott, and R. Roy. Detecting phase synchronization in a chaotic laser array. Phys. Rev. Lett., 87(4):044101, 2001. 
[16] B. Bezruchko, V. Ponomarenko, M. G. Rosenblum, and A. S. Pikovsky. Characterizing direction of coupling from experimental observations. Chaos, 13 (1):179-184, 2003.

[17] I. Z. Kiss, Y. Zhai, and J. L. Hudson. Control of complex dynamics with timedelayed feedback in populations of chemical oscillators: Desynchronization and clustering. Ind. Eng. Chem. Res., 47:3502-3514, 2008.

[18] Y. Zhai, , I. Z. Kiss, and J. L. Hudson. Emerging coherence of oscillating chemical reactions on arrays: Experiments and simulations. Ind. Eng. Chem. Res., 43:315-326, 2004. URL http://pubs.acs.org/doi/full/10.1021/ ie030164z.

[19] I. Z. Kiss, Y. Zhai, and J. L. Hudson. Emerging coherence in a population of chemical oscillators. Science, 296:1676, 2002.

[20] I. Z. Kiss, C. G. Rusin, H. Kori, and J. L. Hudson. Engineering complex dynamical structures: Sequential patterns and desynchronization. Science, 316(5833):1886-1889, 2007.

[21] B. Kralemann, L. Cimponeriu, M. Rosenblum, A. Pikovsky, and R. Mrowka. Uncovering interaction of coupled oscillators from data. Phys. Rev. E, 76: 055201, 2007.

[22] H. Osterhage, F. Mormann, M. Staniek, and K. Lehnertz. Measuring synchronization in the epileptic brain: a comparison of different approaches. Int $J$ Bifurcation Chaos Appl Sci Eng, 17:35393544, 2007.

[23] M. A. Trevisan, S. Bouzat, I. Samengo, and G. B. Mindlin. Dynamics of learning in coupled oscillators tutored with delayed reinforcements. Phys. Rev. E, 72:011907, 2005. 
[24] B. Kralemann, A. Pikovsky, and M. Rosenblum. Reconstructing phase dynamics of oscillator networks. Chaos, 21:025104, 2011.

[25] M. Wickramasinghe, E. M. Mrugacz, and I. Z. Kiss. Dynamics of electrochemical oscillators with electrode size disparity: asymmetrical coupling and anomalous phase synchronization. Phys. Chem. Chem. Phys., 13:15483-15491, 2011.

[26] C. Rocsoreanu, A. Georgescu, and N. Giurgiteanu. The FitzHugh-Nagumo Model: Bifurcation and Dynamics. Kluwer Academic Publishers, Boston, 2000.

[27] E. M. Izhikevich. Dynamical Systems in Neuroscience: The Geometry of Excitability and Bursting. The MIT Press, Cambridge, MA, 2007.

[28] Wikipedia. Pendulum phase portrait - wikipedia, the free encyclopedia, 2013. URL http://upload.wikimedia.org/wikipedia/en/d/da/ Pendulum_Phase_Portrait.jpg. [Online; accessed 15-November-2013].

[29] M. Desroches, J. Guckenheimer, B. Krauskopf, C. Kuehn, H. M. Osinga, and M. Wechselberger. Mixed-mode oscillations with multiple time scales. SIAM Review, 54(2):211288, 2012.

[30] S. M. Baer and T. Erneux. Singular hopf bifurcation to relaxation oscillations. SIAM J. Appl. Math., 46(5):721739, 1986.

[31] C. G. Rusin, S. E. Johnson, J. Kapur, and J. L. Hudson. Engineering the synchronization of neuron action potentials using global time-delayed feedback stimulation. Phys. Rev. E, 84(1):066202, 2011.

[32] S. E. Johnson. Neural Synchronization in Seizures. PhD thesis, University of Virginia, 2012. 
[33] J. Miyazaki and S. Kinoshita. Method for determining a coupling function in coupled oscillators with application to belousov-zhabotinsky oscillators. Phys. Rev. E, 74(5):056209, 2006.

[34] I. Z. Kiss, C. G. Rusin, H. Kori, and J. L. Hudson. On-line supplement, engineering complex dynamical structures: Sequential patterns and desynchronization, see www.sciencemag.org/cgi/content/full/1140858/dc1.

[35] C. G. Rusin. Engineering the Behavior of Complex Rhythmic Systems. PhD thesis, University of Virginia, 2009.

[36] R. J. Williams and N. D. Martinez. Simple rules yield complex food webs. Nature, 404:180-183, 2000.

[37] A. Broder, R. Kumar, F. Maghoul, P. Raghavan, S. Rajagopalan, R. Stata, A. Tomkins, and J. Weiner. Graph structure in the web. Comput. Netw., 33: 309-320, 2000.

[38] Y. Moreno, J. B. Gomez, and A. F. Pacheco. Instability of scale-free networks under node-breaking avalanches. Europhys. Let., 58:630-636, 2002.

[39] O. Diekmann and J. Heesterbeek. Mathematical Epidemiology of Infectious Diseases: Model Building, Analysis and Interpretation. Wiley, New York, 2000.

[40] S. H. Strogatz. Exploring complex networks. Nature, 410:268-276, 2001.

[41] S. Boccaletti, V. Latora, Y. Moreno, M. Chavez, and D. Hwang. Complex networks: Structure and dynamics. Phys. Rep., 424:175-308, 2006.

[42] A. Arenas, A. Diaz-Guilera, J. Kurths, Y. Moreno, and C. Zhou. Synchronization in complex networks. Phys. Rep., 469:93-153, 2008. 
[43] A. Barrat, M. Barthélemy, and A. Vespignani. Dynamical Processes on Complex Networks. Cambridge University Press, Cambridge, 2008.

[44] F. R. K. Chung. Spectral Graph Theory. AMS, Providence, 1994.

[45] S. N. Dorogovtsev, A. V. Goltsev, J. F. F. Mendes, and A. N. Samukhin. Spectra of complex networks. Phys. Rev. E, 68(1):046109, 2003.

[46] M. Rajendran and I. Kevrekidis. Coarse graining the dynamics of heterogeneous oscillators in networks with spectral gaps. Phys. Rev. E, 84(1):036708, 2011.

[47] L. M. Pecora and T. L. Carroll. Master stability functions for synchronized coupled systems. Phys. Rev. Lett., 80(10):2109-2112, 1998.

[48] L. M. Pecora. Synchronization conditions and desynchronizing patterns in coupled limit-cycle and chaotic systems. Phys. Rev. E, 58(1):347-360, 1998.

[49] C.-U. Choe, T. Dahms, P. Hövel, and E. Schöll. Controlling synchrony by delay coupling in networks: From in-phase to splay and cluster states. Phys. Rev. E, 81:1-4, 2010.

[50] L. M. Pecora. Synchronization of oscillators in complex networks. Pramana-J Phys, 70(6):1175-1198, 2008.

[51] M. Barahona and L. M. Pecora. Synchronization in small-world systems. Phys. Rev. Lett., 89(1):1-4, 2002.

[52] D. Haim, O. Lev, L.M. Pismen, and M. Sheintuch. Modeling periodic and chaotic dynamics in anodic nickel dissolution. J. Phys. Chem., 96(6):2676$2681,1992$. 
[53] Y. Zhai. Collective Dynamics of Rhythmic Metal Electrodissolution: Synchronization, Desynchronization, and Clustering. PhD thesis, University of Virginia, 2005.

[54] K. A. Blaha, A. Pikovsky, M. Rosenblum, M. T. Clark, C. Rusin, and J. L. Hudson. Reconstruction of two-dimensional phase dynamics from experiments on coupled oscillators. Phys. Rev. E, 84(1):046201, 2011.

[55] B. Kralemann, L. Cimponeriu, M. Rosenblum, A. Pikovsky, and R. Mrowka. Phase dynamics of coupled oscillators reconstructed from data. Phys. Rev. E, 77(6):066205, 2008. doi: 10.1103/PhysRevE.77.066205.

[56] A. T. Winfree. The Geometry of Biological Time. Springer-Verlag, Berlin, 1980.

[57] A. Pikovsky, M. Rosenblum, and J. Kurths. Synchronization. A Universal Concept in Nonlinear Sciences. Cambridge University Press, Cambridge, 2001.

[58] D. Hansel, G. Mato, and C. Meunier. Clustering and slow switching in globally coupled phase oscillators. Phys. Rev. E, 48(5):3470-3477, 1993.

[59] S. K. Han, C. Kurrer, and Y. Kuramoto. Dephasing and bursting in coupled neural oscillators. Phys. Rev. Let., 75(17):3190-3193, 1995.

[60] H. Daido. Onset of cooperative entrainment in limit-cycle oscillators with uniform all-to-all interactions: bifurcation of the order function. Physica D (Amsterdam), 91:24, 1996.

[61] S. H. Strogatz. From Kuramoto to Crawford: Exploring the onset of synchronization in populations of coupled oscillators. Physica D, 143(1-4):1-20, 2000. 
[62] I. Z. Kiss, Y. Zhai, and J. L. Hudson. Predicting mutual entrainment of oscillators with experiment-based phase models. Phys. Rev. Lett., 94(24):4, 2005.

[63] Y. Zhai, I. Z. Kiss, and J. L. Hudson. Amplitude death through a hopf bifurcation in coupled electrochemical oscillators: Experiments and simulations. Phys. Rev. E, 69(2):7, 2004.

[64] M. K. S. Yeung and S. H. Strogatz. Time delay in the Kuramoto model of coupled oscillators. Phys. Rev. Lett., 82(3):648-651, 1999.

[65] E. Montbrió, D. Pazó, and J. Schmidt. Time delay in the kuramoto model with bimodal frequency distribution. Phys. Rev. E, 74(5):056201, 2006. doi: 10.1103/PhysRevE.74.056201.

[66] E. Rodriguez, N. George, J.P. Lachaux, J. Martinerie, B. Renault, and F.J. Varela. Perception's shadow: long-distance synchronization of human brain activity. Nature, 397(6718):430-433, 1999.

[67] B. Kralemann, M. Rosenblum, and A. Pikovsky. On-line toolbox, see www.agnld.uni-potsdam.de/ mros/damoco.html.

[68] M. Rosenblum and A. Pikovsky. Detecting direction of coupling in interacting oscillators. Phys. Rev. E, 64(4):045202(R), 2001.

[69] K. A. Blaha, J. Lehnert, A. Keane, T. Dahms, P. Hoevel, E. Schöll, and J. L. Hudson. Clustering in delay-coupled smooth and relaxational chemical oscillators. submitted, 2013.

[70] R. Albert and A.-L. Barabási. Statistical mechanics of complex networks. Rev. Mod. Phys., 74:47, 2012. 
[71] E. Schöll and H. G. Schuster. Handbook of Chaos Control: second completely revised and enlarged edition. Wiley-VCH, Weinheim, 2008.

[72] E. Schöll. Chapter 4. In Q. Ding J.-Q. Sun, editor, Advances in Analysis and Control of Time-Delayed Dynamical Systems. World Scientific, Singapore, 2013.

[73] D. Pazó, N. Montejo, and V. Pérez-Muñuzuri. Wavefronts and spatio-temporal chaos in an array of coupled lorenz oscillators. Phys. Rev. E, 63:066206, 2001.

[74] V. Dziubak, Y. L. Maistrenko, and E. Schöll. Coherent traveling waves in nonlocally coupled chaotic systems. Phys. Rev. E, 87:032907, 2001.

[75] F. Sorrentino and E. Ott. Network synchronization of groups. Phys. Rev. E, 76: 056114, 2007.

[76] T. Dahms, J. Lehnert, and E. Schöll. Cluster and group synchronization in delay-coupled networks. Phys. Rev. E, 86:016202, 2012.

[77] P. S. Skardal, E. Ott, and J. Restrepo. Cluster synchrony in systems of coupled phase oscillators with higher-order coupling. Phys. Rev. E, 84(1):036208, 2011.

[78] J. Kestler, W. Kinzel, and I. Kanter. Chaos synchronization with dynamic filters: Two way is better than one way. Phys. Rev. E, 76(1):035202, 2007.

[79] J. Kestler, E. Kopelowitz, I. Kanter, and W. Kinzel. Patterns of chaos synchronization. Phys. Rev. E, 77(1):046209, 2008.

[80] C. U. Choe, H. Jang, V. Flunkert, T. Dahms, P. Hövel, and E. Schöll. Stabilization of periodic orbits near a subcritical hopf bifurcation in delay-coupled networks. Dyn. Sys., 28(1):1, 2013. 
[81] Y. Aviad, I. Reidler, M. Zigzag, M. Rosenbluh, and I. Kanter. Coupled lasers: phase versus chaos synchronization. Opt. Express, 20(1):4352, 2012.

[82] R. Vardi, A. Wallach, E. Kopelowitz, M. Abeles, S. Marom, and I. Kanter. Synthetic reverberating activity patterns embedded in networks of cortical neurons. Europhys. Lett., 97(1):066002, 2012.

[83] M. R. Tinsley, S. Nkomo, and K. Showalter. Chimera and phase-cluster states in populations of coupled chemical oscillators. Nature Physics, 8(1):662, 2012.

[84] C. R. S. Williams, T. E. Murphy, R. Roy, F. Sorrentino, T. Dahms, and E. Schöll. Experimental observations of group synchrony in a system of chaotic optoelectronic oscillators. Phys. Rev. Lett., 110:064104, 2013.

[85] D. P. Rosin, D. Rontani, D. J. Gauthier, and E. Schöll. Control of synchronization patterns in neural-like boolean networks. Phys. Rev. Lett., 110(1):104102, 2013.

[86] C. U. Choe, T. Dahms, P. Hövel, and E. Schöll. Proceedings of the eighth aims international conference on dynamical systems, differential equations and applications. In American Institute of Mathematical Sciences, pages 292-301, Springfield, MO, USA, September 2011.

[87] C. G. Rusin, H. Kori, I. Z. Kiss, and J. L. Hudson. Synchronization engineering: tuning the phase relationship between dissimilar oscillators using nonlinear feedback. Philosophical Transactions of the Royal Society A: Mathematical, Physical and Engineering Sciences, 368(1918):2189-2204, 2010.

[88] K. Engelborghs, T. Luzyanina, and G. Samaey. Technical Report No. TW-330, Department of Computer Science, K.U.Leuven, Belgium, 
http://www.cs.kuleuven.be/publicaties/rapporten/tw/tw330.abs.html, 2001.

[89] K. Engelborghs, T. Luzyanina, and D. Roose. Numerical bifurcation analysis of delay differential equations using dde-biftool. In ACM Transactions on Mathematical Software, volume 28, page 1, 2002.

[90] C. Otto, B. Globisch, K. Lüdge, E. Schöll, and T. Erneux. Complex dynamics of semiconductor quantum dot lasers subject to delayed optical feedback. Int. J. Bifurcation Chaos, 22:1250246, 2012.

[91] S. Watanabe and S. H. Strogatz. Constants of motion for superconducting Josephson arrays. Physica D, 74:197-253, 1994.

[92] W. Braun, A. Pikovsky, M. A. Matias, and P. Colet. Global dynamics of oscillator populations under common noise. EPL, 99:20006, 2012.

[93] S. Yamaguchi, H. Isejima, T. Matsuo, R. Okura, K. Yagita, M. Kobayashi, and H. Okamura. Synchronization of cellular clocks in the suprachiasmatic nucleus. Science, 302:1408, 2003.

[94] U. Abraham, A. E. Granada, P. O. Westermark, M. Heine, A. Kramer, and H. Herzel. Coupling governs entrainment range of circadian clocks. Molec. Sys. Biol., 6:438, 2010.

[95] F. X. Witkowski, L. J. Leon, P. A. Penkopske, W. R. Giles, M. L. Spano, W. L. Ditto, and A. T. Winfree. Spatiotemporal evolution of ventricular fibrillation. Nature, 392:78, 1998.

[96] G. B. Ermentrout. The mathematics of biological oscillators. In M. L. Johnson, 
editor, Essential Numerical Computer Methods. Academic Press, Waltham, Massachusetts, 2010.

[97] B. Kralemann, M. Frühwirth, A. Pikovsky, M. Rosenblum, T. Kenner, J. Schaefer, and M. Moser. In vivo cardiac phase response curve elucidates human respiratory heart rate variability. Nature Communications, 4:2418, 2013.

[98] I. Z. Kiss, Y. Zhai, and J. L. Hudson. Collective dynamics of a weakly coupled electrochemical reaction on an array. Ind. Eng. Chem. Res., 41:6363, 2002.

[99] Y. Kuramoto and D. Battogtokh. Coexistence of coherence and incoherence in nonlocally coupled phase oscillators. Nonlinear Phenom. Complex Syst., 5 (1):380, 2002.

[100] D. M. Abrams and S. H. Strogatz. Chimera states for coupled oscillators. Phys. Rev. Lett., 93:174102, 2004.

[101] D. Abrams and S. Strogatz. Chimera states in a ring of non locally coupled oscillators. Int. J. Bifurcat. Chaos, 16(1):1201, 2006.

[102] O. Omelchenko, M. Wolfrum, and Y. Maistrenko. Chimera states as chaotic spatiotemporal patterns. Phys. Rev. E, 81(1):065201, 2010.

[103] C. Laing. Chimera states in heterogeneous networks. Chaos, 19(1):1216, 2010.

[104] H. Hong and S. Strogatz. Conformists and contrarians in a kuramoto model with identical natural frequencies. Phys. Rev. E, 84(1):046202, 2011.

[105] H. Hong and S. Strogatz. Kuramoto model of coupled oscillators with positive and negative coupling parameters: An example of conformist and contrarian oscillators. Phys. Rev. Lett., 106(1):054102, 2011. 


\section{List of Publications Based on the}

\section{Dissertation}

1. K. A. Blaha, A. Pikovsky, M. Rosenblum, M. T. Clark, C. G. Rusin and J. L. Hudson, "Reconstruction of two-dimensional phase dynamics from experiments on coupled oscillators” Phys. Rev. E, 84, 046201, 2011.

2. K. A. Blaha, J. Lehnert, A. Keane, T. Dahms, P. Hövel, E. Schöll and J. L. Hudson, "Clustering in delay-coupled smooth and relaxational chemical oscillators" Phys. Rev. E, (accepted).

3. K. A. Blaha, J. Lehnert, A. Keane, P. Hövel, I. Z. Kiss, E. Schöll, J. L. Hudson, "Exploring a phase and radius model for coupled experimental relaxational oscillators" (paper in preparation). 